Pacific Northwest

National Laboratory

Operated by Battelle for the

U.S. Department of Energy

\title{
Effects of Globally Waste-Disturbing Activities on Gas Generation, Retention, and Release in Hanford Waste Tanks
}

\author{
C.W. Stewart \\ J.L. Huckaby \\ P.A. Meyer
}

July 2003

Prepared for the U.S. Department of Energy under Contract DE-AC06-76RL01830 


\section{DISCLAIMER}

This report was prepared as an account of work sponsored by an agency of the United States Government. Neither the United States Government nor any agency thereof, nor Battelle Memorial Institute nor any of their employees makes any warranty, express or implied, or assumes any legal liability or responsibility for the accuracy, completeness, or usefulness of any information, apparatus, product, or process disclosed or represents that its use would not infringe privately owned rights. Reference herein to any specific commercial product, process, or service by trade name, trademark, manufacturer, or otherwise does not necessarily constitute or imply its endorsement, recommendation, or favoring by the United States Government or any agency thereof, or Battelle Memorial Institute. The views and opinions of authors expressed herein do not necessarily state or reflect those of the United States Government or any agency thereof.

\section{PACIFIC NORTHWEST NATIONAL LABORATORY \\ operated by \\ BATTELLE \\ for the \\ UNITED STATES DEPARTMENT OF ENERGY \\ under Contract DE-AC06-76RL01830}

Printed in the United States of America

Available to DOE and DOE contractors from the

Office of Scientific and Technical Information,

P.O. Box 62, Oak Ridge, TN 37831-0062;

ph: (865) 576-8401

fax: (865) $576-5728$

email: reports@adonis.osti.gov

Available to the public from the National Technical Information Service,

U.S. Department of Commerce, 5285 Port Royal Rd., Springfield, VA 22161

ph: (800) 553-6847

fax: (703) 605-6900

email: orders@ntis.fedworld.gov

online ordering: http://www.ntis.gov/ordering.htm

This document was printed on recycled paper.

$(8 / 00)$ 


\section{Effects of Globally Waste-Disturbing Activities on Gas Generation, Retention, and Release in Hanford Waste Tanks}

CW Stewart

JL Huckaby

PA Meyer

July 2003

Prepared for

the U.S. Department of Energy

under Contract DE-AC06-76RL01830

Pacific Northwest National Laboratory

Richland, WA 99352 


\begin{abstract}
Various operations are authorized in Hanford single- and double-shell tanks that disturb all or a large fraction of the waste. These globally waste-disturbing activities have the potential to release a large fraction of the retained flammable gas and to affect future gas generation, retention, and release behavior. This report presents analyses of the expected flammable gas release mechanisms and the potential release rates and volumes resulting from these activities. The background of the flammable gas safety issue at Hanford is summarized, as is the current understanding of gas generation, retention, and release phenomena. Considerations for gas monitoring and assessment of the potential for changes in tank classification and steady-state flammability are given.
\end{abstract}




\section{Summary}

This report presents an assessment of the effects of globally waste-disturbing activities on flammable gas generation, retention, and release. The assessment includes the potential for hazardous flammable gas releases, considerations for determining the frequency of gas monitoring during the disturbance, and the potential for long-term changes in tank classification and gas generation rate resulting from the disturbance.

Global waste disturbances are those that affect all or a large fraction of the waste in a tank. The disturbance may involve draining the supernatant or interstitial liquid, dissolving solids, or mobilizing the settled sediment. Nine specific activities that occur or are approved in Hanford double-shell (DST) and single-shell (SST) waste tanks are considered in this report. While many of these operations could be performed in both SSTs and DSTs, all but one are considered specific to one type. The nine globally waste-disturbing activities are:

- Waste removal - pumping supernatant or slurry out of a DST

- Waste addition - pumping liquid or slurry into a DST

- Saltwell pumping — removing interstitial liquid from SSTs

- Saltcake dissolution - adding water to dissolve soluble solids in an SST while pumping out the resulting brine

- Water addition - adding water to a DST or SST without concurrent brine removal

- Mixer pump operation-mobilizing sediment in DSTs by the hydraulic jet forces of a mixer pump

- Airlift circulator operation - mobilizing sediment by airlift pumping in several DSTs

- Chemical addition - adding sodium hydroxide or nitrite solution to DSTs to prevent corrosion

- Evaporation - gradually concentrating supernatant in DSTs by evaporating water from the waste surface.

The considerations for each of these operations depend on the waste group classification of the tank. Three waste groups are defined based on the potential for flammability and whether the tank exhibits buoyant displacement gas release events (BDGREs). Waste Group A and B tanks store sufficient gas to make the headspace flammable if all of it were suddenly released; Waste Group C tanks do not. Waste Group A tanks exhibit spontaneous BDGREs, while tanks in Waste Group B do not. Tanks are assigned to waste groups based on several criteria for BDGRE behavior and the waste configuration and volume.

A waste-disturbing activity can cause or influence gas releases in three ways: 1) BDGREs in Waste Group A tanks may be amplified by decreasing the headspace and increasing the hydrostatic pressure on the gas (e.g., by adding waste, water, or chemicals), 2) BDGREs can be induced during the operation as a result of lowering the neutral buoyancy gas fraction by suspending sediment in the supernatant (e.g., by mixer pump or airlift circulator operation) or 
otherwise increasing the supernatant density (e.g., by waste or chemical additions), and 3) the waste disturbance itself can produce gas releases that can eventually make the headspace flammable if the ventilation rate is low (e.g., saltwell pumping, saltcake dissolution). The main concern is for operations in Group A and B tanks. Though gas releases occur in Group C tanks, they are inconsequential because these tanks do not have enough retained gas to make their headspaces flammable.

Besides releasing gas, a waste disturbance can also change a tank's waste group classification in the long term. A Group C tank experiencing BDGREs (e.g., SY-103) can move all the way into Group A by a waste addition that reduces its headspace sufficiently. Any operation that makes the supernatant denser can potentially decrease the neutral buoyancy gas fraction, initiate BDGREs, and move a tank from Group B to Group A. However, initiating BDGREs in a Group $\mathrm{C}$ tank will not change its classification. Waste, water, and chemical addition prove to be the only activities capable of moving a tank to a higher waste group. Conversely, any of the waste removal activities could move a tank to Group C.

The hydrogen generation rate depends both on the amount and temperature of the waste, specifically the liquid portion, and the concentration of dissolved salts and total organic carbon in the liquid. Therefore, waste addition clearly causes an increase in the hydrogen generation rate, and any major removal of waste, especially liquid, will decrease it. Water or caustic additions (that do not dissolve a large volume of solids) do not change the hydrogen generation rate appreciably. Waste temperature can increase considerably from energy dissipation by mixer pump operation. Because hydrogen generation is sensitive to temperature, a relatively short period of continuous mixer pump operation could easily increase the hydrogen generation rate by an order of magnitude. If the waste temperature remains elevated for an extended period, the hydrogen generation rate and steady-state flammability hazard of the tank should be evaluated at the new temperature. 


\section{Contents}

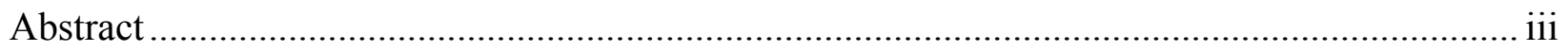

Summary

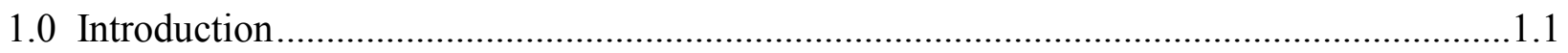

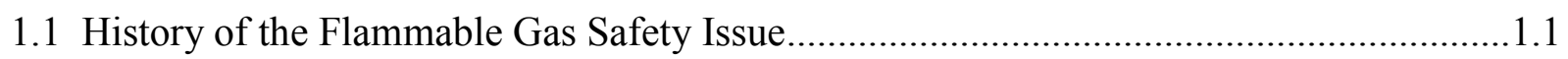

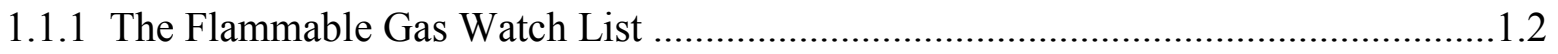

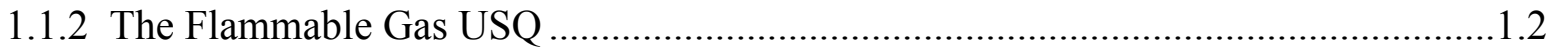

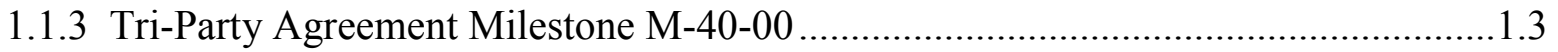

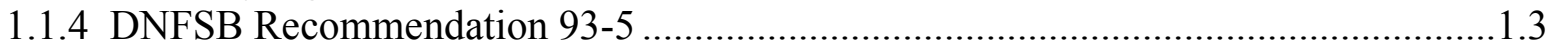

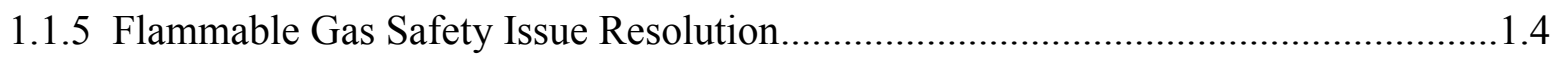

1.2 Local and Global Waste Disturbances .............................................................................

1.2.1 Gas Releases Observed During Local Disturbances...................................................

1.2.2 Gas Releases Observed During Global Waste Disturbances ......................................1.5

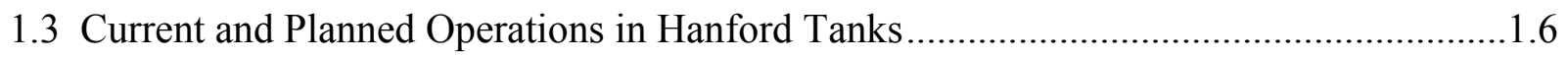

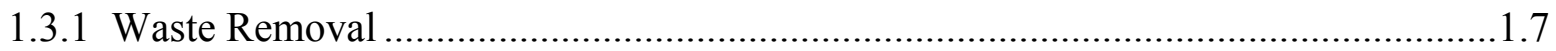

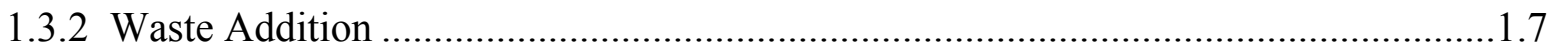

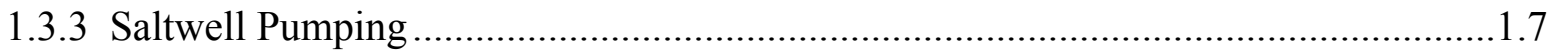

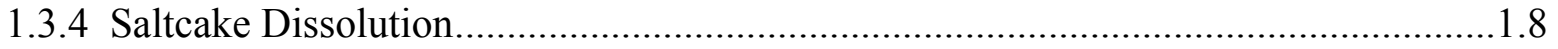

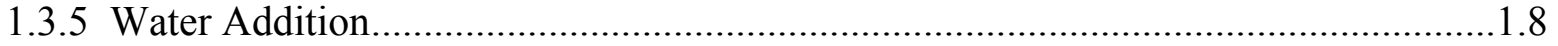

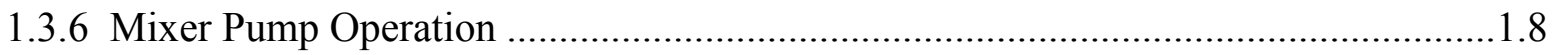

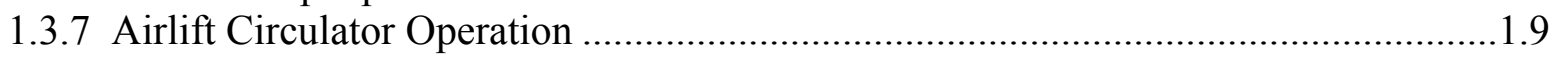

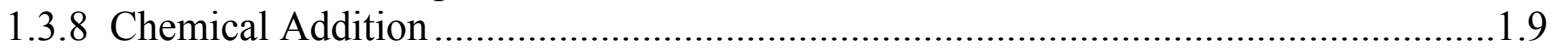

1.3.9 Natural Evaporation ....................................................................................... 1.9

2.0 Physics of Gas Generation, Retention, and Release .......................................................... 2.1

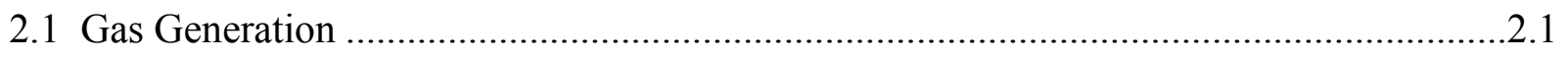

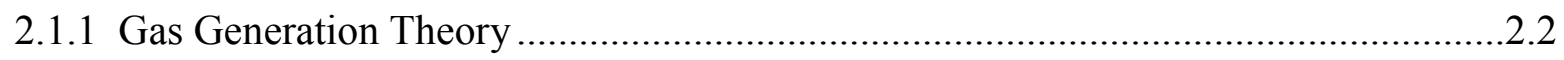

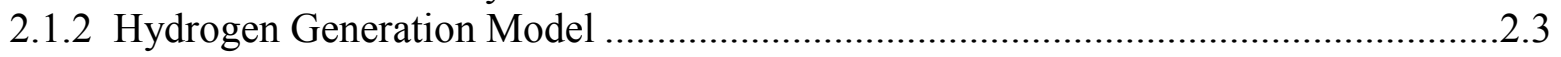

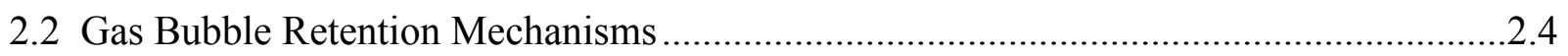

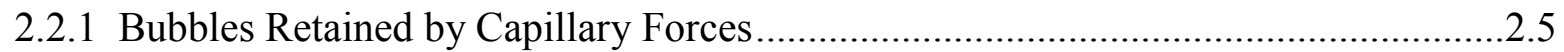

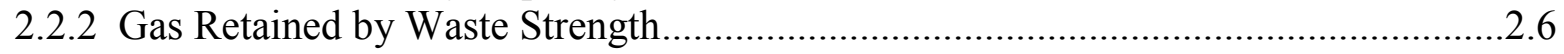

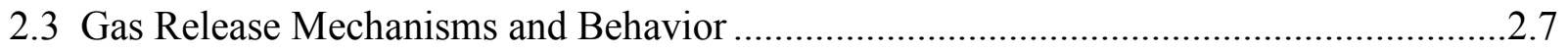

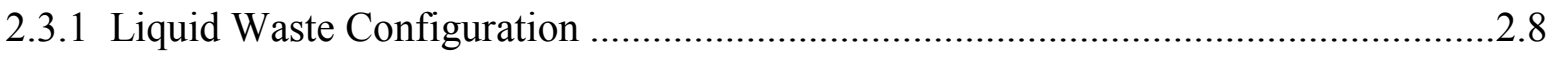

2.3.2 Liquid-over-Solid Configuration .................................................................................

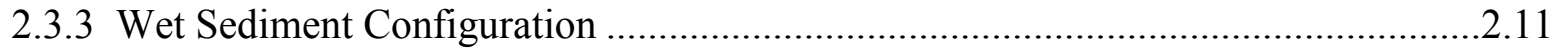

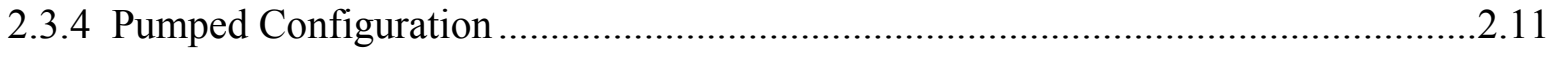

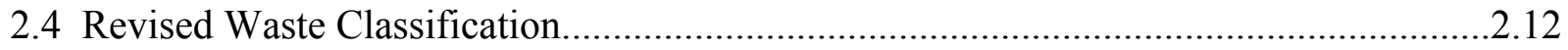

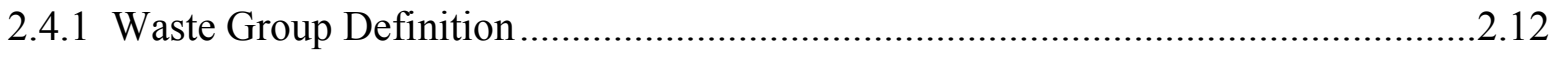

2.4.2 Criteria for Waste Group Classification ..................................................................... 
3.0 Gas Releases Induced by Global Waste Disturbances...................................................

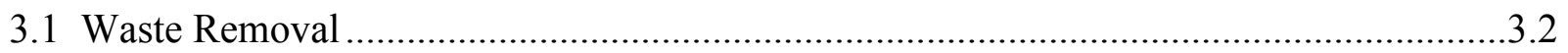

3.1.1 Mechanisms for Gas Releases During Waste Removal.............................................2.2

3.1.2 Expected Gas Releases During Waste Removal........................................................

3.1.3 Gas Monitoring Considerations During Waste Removal ...........................................

3.1.4 Potential Changes in Waste Group Classification After Waste Removal ..................3.7

3.1.5 Potential Changes in Hydrogen Generation After Waste Removal...........................3.7

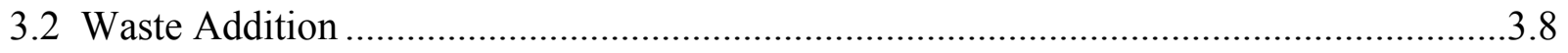

3.2.1 Mechanisms for Gas Releases During Waste Addition ........................................... 3.8

3.2.2 Expected Gas Releases During Waste Addition..................................................... 12

3.2.3 Gas Monitoring Considerations During Waste Addition...........................................13

3.2.4 Potential Changes in Waste Classification After Waste Addition...........................3.14

3.2.5 Potential Changes in Hydrogen Generation After Waste Addition ..........................3.16

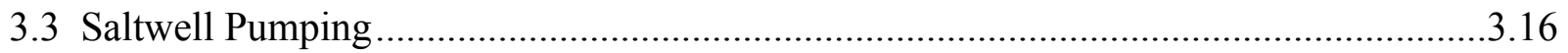

3.3.1 Mechanisms for Gas Releases During Saltwell Pumping........................................17

3.3.2 Expected Gas Releases During Saltwell Pumping..................................................18

3.3.3 Gas Monitoring Considerations During Saltwell Pumping ...................................3.20

3.3.4 Potential Changes in Waste Classification After Saltwell Pumping ........................2.22

3.3.5 Potential Changes in Hydrogen Generation After Saltwell Pumping.......................3.22

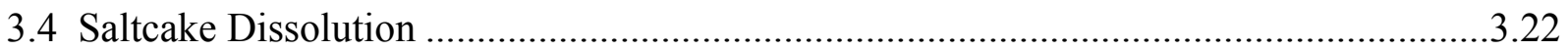

3.4.1 Mechanisms for Gas Release During Saltcake Dissolution..................................3.22

3.4.2 Expected Gas Releases During Saltcake Dissolution .........................................23

3.4.3 Gas Monitoring Considerations During Saltcake Dissolution .................................24

3.4.4 Potential Changes in Waste Classification After Saltcake Dissolution ....................3.26

3.4.5 Potential Changes in Hydrogen Generation After Saltcake Dissolution ..................3.26

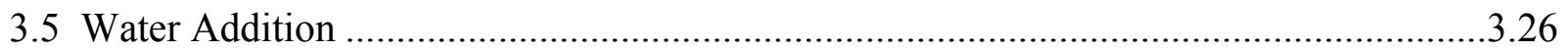

3.5.1 Mechanisms for Gas Release During Water Addition............................................2.27

3.5.2 Expected Gas Releases During Water Addition .......................................................2.27

3.5.3 Gas Monitoring Considerations During Water Addition ........................................2.28

3.5.4 Potential Changes in Waste Classification After Water Addition ............................3.28

3.5.5 Potential Changes in Hydrogen Generation After Water Addition ..........................3.31

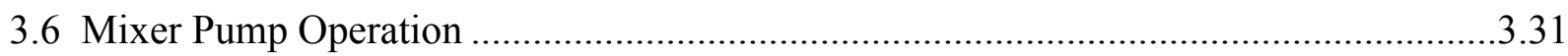

3.6.1 Mechanisms for Gas Releases During Mixer Pump Operation .............................3.31

3.6.2 Expected Gas Releases During Mixer Pump Operation ........................................3.32

3.6.3 Gas Monitoring Considerations During Mixer Pump Operation..............................3.35

3.6.4 Potential Changes in Tank Classification After Mixing ........................................3.36

3.6.5 Potential Changes in Hydrogen Generation After Mixing …................................3.37

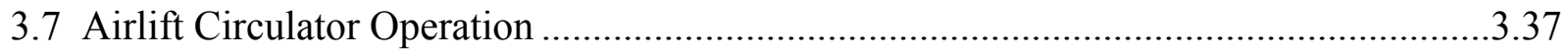

3.7.1 Mechanisms for Gas Releases During ALC Operation ........................................3.37

3.7.2 Expected Gas Releases During ALC Operation ...............................................3.39

3.7.3 Gas Monitoring Considerations During ALC Operation............................................45

3.7.4 Potential Changes in Tank Classification After ALC Operation...............................45

3.7.5 Potential Changes in Hydrogen Generation After ALC Operation ............................4.45 


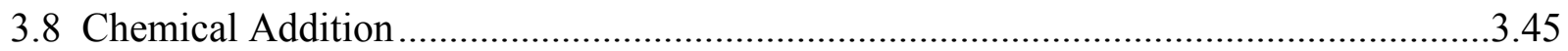

3.8.1 Mechanisms for Gas Release During Chemical Addition .........................................4.

3.8.2 Expected Gas Releases During Chemical Addition....................................................4.

3.8.3 Gas Monitoring Considerations During Chemical Addition .....................................3.46

3.8.4 Potential Changes in Waste Classification After Chemical Addition .......................3.47

3.8.5 Potential Changes in Hydrogen Generation After Chemical Addition..........................47

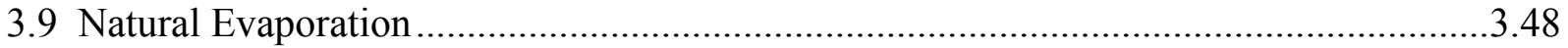

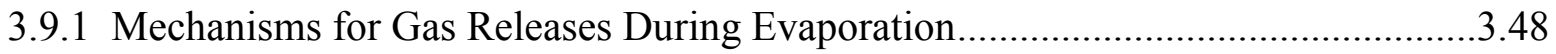

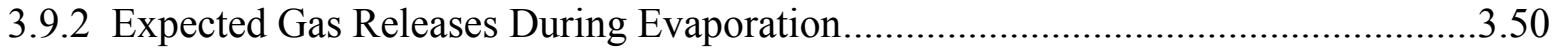

3.9.3 Gas Monitoring Considerations During Evaporation ...............................................5.

3.9.4 Potential Changes in Tank Classification After Evaporation ......................................5.

3.9.5 Potential Changes in Hydrogen Generation after Evaporation..................................3.53

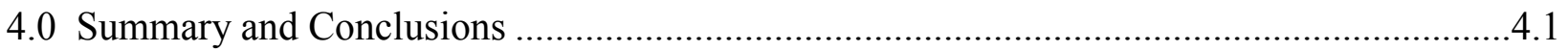

4.1 Potential for Significant Gas Releases.......................................................................... 4.1

4.2 Potential Changes in Waste Group Classification ..........................................................

4.3 Potential Changes in Hydrogen Generation......................................................................4.4

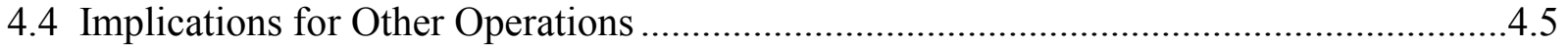

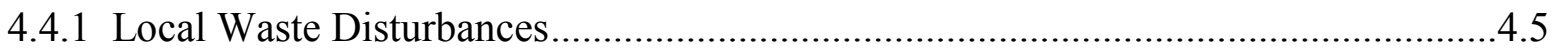

4.4.2 Other Global Waste Disturbances...........................................................................

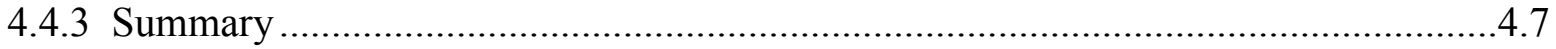

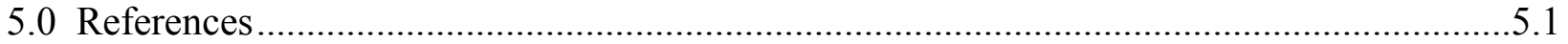




\section{Figures}

3.1 Sensitivity of Peak Hydrogen Concentration to Ventilation Rate in AN-105 .................. 3.8

3.2 Mechanism for Inducing BDGREs During Waste Addition (AN-107).......................... 3.9

3.3 Tank Waste Configuration During and After Waste Transfer..................................... 3.10

3.4 Effects of Waste Addition on Buoyancy Ratio for Tank AW-105 …............................ 3.15

3.5 Effects of Waste Addition on Buoyancy Ratio for Tank AN-107 ................................ 3.15

3.6 U-105 Hydrogen Release and Saltwell Pumping Rates................................................. 3.18

3.7 Tank 241-S-106 Estimated Hydrogen Release Rate and Pumping Rate ........................ 3.19

3.8 U-105 Ammonia Release and Waste Volume Pumped ................................................ 3.20

3.9 Hydrogen Concentration and Waste Level History in S-111 ...................................... 3.21

3.10 Hydrogen Concentration Versus Time for Saltcake Dissolution; Group B Tank ............ 3.25

3.11 Required Gas Monitoring Frequency Versus Brine Pumping Rate.............................. 3.26

3.12 Sensitivity of Hydrogen Concentration to Ventilation in AN-103 During Mixing .......... 3.36

3.13 Schematic of Typical Airlift Circulator Operation ...................................................... 3.38

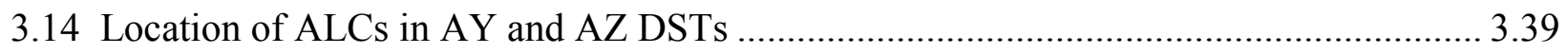

3.15 Stages of Waste Disturbance During ALC Operation ................................................ 3.40

3.16 ALC Flow Rate and Velocity for Tank AZ-102 ........................................................ 3.43

3.17 Gas Release Rate from a Single ALC in Tank AZ-102 ............................................... 3.44

3.18 Tank Waste Configuration Before and After Evaporation ........................................... 3.51

3.19 Effects of Evaporation on Buoyancy Ratio ................................................................ 3.51

3.20 Water Partial Pressure Versus Solution Density..................................................... 3.52

\section{Tables}

3.1 Summary of Globally Waste-Disturbing Activities.................................................. 3.1

3.2 Summary of Supernatant Decant Analysis Results ....................................................... 3.4

3.3 Parameters Used for Waste Transfer Analysis .......................................................... 3.16

3.4 Peak Hydrogen Concentrations After Water and Caustic Addition ............................... 3.30

3.5 Time to Return to BDGRE Behavior After Degassing.............................................. 3.32

3.6 Summary of Mixing Gas Release Analysis Results ................................................... 3.34

3.7 Waste Properties in Tanks with ALCs..................................................................... 3.42

3.8 Disturbed Sediment Volumes ................................................................................ 3.42

3.9 Change in Buoyancy Ratio for Addition of $100 \mathrm{kgal}$ at $\mathrm{SpG}=1.5$............................ 3.47

3.10 Tank Parameters Used for Evaporation Analysis ....................................................... 3.51

4.1 Potential Gas Releases During Globally Waste-Disturbing Activities ............................ 4.2

4.2 Potential Changes in Waste Group from Globally Waste-Disturbing Activities .............. 4.3

4.3 Changes in Gas Generation from Globally Waste-Disturbing Activities ......................... 4.4 


\subsection{Introduction}

Various operations are authorized in Hanford high-level radioactive waste storage tanks that disturb a large fraction of the waste. These "globally waste-disturbing activities" are of interest because they are now being performed or are planned as part of the Hanford mission to retrieve waste from the older single-shell tanks (SSTs), transfer it to the newer double-shell tanks (DSTs), and deliver waste feed from the DSTs to the vitrification plant for treatment. Some of the activities, such as saltwell pumping, have been ongoing for many years. Others, like supernatant decanting, have been proposed only recently.

Most of the waste tanks retain some volume of flammable gas, and some tanks retain a substantial gas volume. Because globally waste-disturbing activities potentially can release a large fraction of that gas quickly, all such operations must be approached with care. The potential gas releases from these kinds of activities have long been subject to intense scrutiny brought about by the Flammable Gas Safety Issue. However, that safety issue has been formally closed along with the associated Unreviewed Safety Questions (USQ), and the remaining tanks were removed from the Flammable Gas Watch List on August 17, 2001. These achievements now permit a general update of the Tank Farm Final Safety Analysis Report (Cash 2000), Tank Farm Technical Safety Requirements (CHG 2001), and administrative controls governing tank farm operations.

This report presents an assessment of the predicted effects of globally waste-disturbing activities on flammable gas generation, retention, and release in support of this update. The assessment includes the potential for hazardous flammable gas releases, considerations for determining the frequency of gas monitoring during the disturbance, and the potential for longterm changes in tank classification and gas generation rate resulting from the disturbance.

The rest of the Introduction sets the stage for a detailed discussion of each globally wastedisturbing activity. Section 1.1 summarizes the history of the Flammable Gas Safety Issue from inception to closure. Section 1.2 describes the difference between global and local waste disturbances and the history of gas release observations for each type. Section 1.3 introduces the globally waste-disturbing activities to be assessed for gas releases.

The mechanisms for gas generation, retention, and release for the major Hanford waste types and waste configurations are reviewed, and the revised tank waste group classifications and criteria are outlined in Section 2. The potential for significant gas releases for each activity, gasmonitoring issues, and potential changes in tank classification and gas generation rate are discussed in Section 3. Section 4 summarizes our conclusions, and references are listed in Section 5. The Checklist for Technical Peer Review is attached as an appendix.

\subsection{History of the Flammable Gas Safety Issue}

All radioactive wastes in the Hanford underground storage tanks slowly generate a gas mixture that typically includes hydrogen, nitrous oxide, ammonia, nitrogen, and traces of methane and other organic compounds. In most tanks, the gas evolves from the waste into the 
tank headspace at the same rate as it is generated, so the existing active and passive ventilation systems can keep the concentration far below the lower flammability limit (LFL). However, some tanks may retain enough gas within the waste to cause worker injury or damage to equipment if it were suddenly released into the headspace and ignited. Gas releases may occur spontaneously or be induced by external forces (e.g., severe earthquakes) or waste intrusion activities (e.g., core sampling, equipment installation, liquid removal operations). The potential for spontaneous releases and their undesirable consequences constituted the Flammable Gas Safety Issue.

The Flammable Gas Safety Issue was born in 1990 when large gas releases were observed in Tank SY-101. Since then this issue has been documented or acknowledged in the "Watch List," several USQs, a major milestone of the Tri-Party Agreement (Ecology et al. 1996), and a Defense Nuclear Facilities Safety Board (DNFSB) recommendation, as summarized below. A complete discussion of the evolution and closure of the Flammable Gas Safety Issue is given by Johnson et al. (2001).

\subsubsection{The Flammable Gas Watch List}

In November 1990, Public Law 101-510 was passed. Section 3137 of this law, also known as the Wyden Amendment, required the Secretary of Energy to identify the high-level nuclear waste tanks that may have "serious potential for release of high-level waste due to uncontrolled increases in temperature or pressure."

Twenty-three tanks were identified in 1991 by a variety of criteria in response to the public law, and in 1993 two more tanks were added for a total of 25 on the Watch List. Tank SY-101 was removed from the list in January 2001. The remaining 24 tanks, A-101, AN-103, AN-104, AN-105, AX-101, AX-103, AW-101, S-102, S-111, S-112, SX-101, SX-102, SX-103, SX-104, SX-105, SX-106, SX-109, SY-103, T-110, U-103, U-105, U-107, U-108, and U-109, were removed in August 2001. These tanks were also the subject of TPA Milestone M-40-00.

\subsubsection{The Flammable Gas USQ}

An unreviewed safety question (USQ) exists when DOE determines that the nuclear safety Authorization Basis governing the activities at a facility or site may not adequately protect the health and safety of the public, workers, or the environment. DOE declared a USQ in 1990 because the existing controls and analyses did not consider the observed gas releases in SY-101 and postulated phenomena in other tanks. In June 1996, DOE-RL closed the USQ for SY-101 because the mixer pump had mitigated the spontaneous gas release hazard. ${ }^{\text {(a) }}$

The original USQ was updated in July 1996, consolidating earlier determinations into one overall flammable gas USQ determination that was adopted by DOE-RL on November 1,

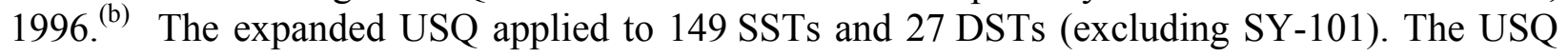

(a) Wagoner JD. June 21, 1996. Closure of Hanford Tank 241-SY-101 Flammable Gas Unreviewed Safety Question (USQ). Letter 96-WSD-060 to AL Trego, Westinghouse Hanford Company, Richland, WA.

(b) Wagoner JD. November 1, 1996. The U.S. Department of Energy (DOE) Richland Operations Office (RL) Definition and Declaration of Flammable Gas Unreviewed Safety Question (USQ). Letter 96-WSD283 to HJ Hatch, Fluor Daniel Hanford Co., Richland, WA. 
for AX-104 was closed by DOE-RL in April $1998^{(\mathrm{a})}$ and for all the other tanks in September $1998^{(\mathrm{b})}$ based on the adequacy of flammability, ignition, and monitoring controls implemented as part of the Authorization Basis. Another USQ (TF-97-0975) was declared for Tank SY-101 in 1998 because of an unexpected rise in the waste level. ${ }^{(c)}$ This USQ was closed in November $2000 .^{(d)}$

\subsubsection{Tri-Party Agreement Milestone M-40-00}

The Tri-Party Agreement (Ecology et al. 1996) was established between the Washington State Department of Ecology, the U.S. Environmental Protection Agency, and DOE in 1989. TPA Milestone M-40-00, "Mitigate/Resolve Tank Safety Issues for High Priority Watch List Tanks," was among the specific milestones established for the various programs at the Hanford Site. This milestone, established in January 1994 with a due date of September 30, 2001, required that corrective action plans be developed for the watch list tanks and that mitigation activities, if required, be implemented to ensure safe storage of waste until retrieval began for treatment and/or disposal operations.

The safety issues for ferrocyanide and organic/nitrate were resolved by showing that these compounds did not exist or that their concentrations were so low that self-sustaining propagating chemical reactions were not plausible. The high-heat issue was limited to one SST (C-106) and was resolved by transferring most of the waste to a DST (AY-102). The flammable gas issue was quite different in that flammable gases are present in all of the waste tanks. However, evaluations showed that the hazard could be managed by following the established control process. When the Flammable Gas Safety Issue was closed in August 2001, the TPA milestone was completed one month ahead of schedule.

\subsubsection{DNFSB Recommendation 93-5}

In July 1993, the DNFSB transmitted Recommendation 93-5 (Conway 1993) on the Hanford Waste Tank Characterization Studies to DOE. Recommendation 93-5 noted that 1) technical information available for the Hanford tank waste was insufficient to ensure that wastes could be safely stored and associated operations could be conducted safely and 2) the characterization effort should be upgraded and expedited.

(a) Wagoner JD. April 21, 1998. Closure of Flammable Gas Unreviewed Safety Question (USQ) for Tank 241-AX-104. Letter 98-TWR-011 to HJ Hatch, Fluor Daniel Hanford, Richland, WA.

(b) Wagoner JD. September 25, 1998. Closure of Flammable Gas Unreviewed Safety Question (USQ) for Tank Waste Remediation System (TWRS) Single-Shell Tanks (SST) and Double-Shell Tanks (DST). Letter 98-SCD-111 to RD Hanson, Fluor Daniel Hanford, Richland, WA.

(c) Kinzer JE. February 26, 1998. Declaration of an Unreviewed Safety Question (USQ) Related to Waste Surface Level Changes in Tank 241-SY-101. Letter 98-WSD-070 to HJ Hatch, Fluor Daniel Hanford, Richland, WA.

(d) Boston HL. November 30, 2000. Approval of Authorization Basis (AB) Amendment for Closure of Tank 241-SY-101 Waste Surface Change Unreviewed Safety Question (USQ) and Removal of Requirement to Operate Tank Mixer Pump. Letter 00-SHD-124 to MP DeLozier, CHG, Richland, WA. 
The DNFSB accepted implementation plans (DOE/RL 1996) that focused characterization efforts on understanding safety-related phenomena to expedite resolution of waste tank safety issues. The plan included 12 deliverables associated with the Flammable Gas Safety Issue. All items, including the ones for flammable gas, were completed and the DNSFB closed 93-5 in November 1999 (Conway 1999). ${ }^{\text {(a) }}$ Satisfactory resolution of DNFSB 93-5 was basic to resolution of the Flammable Gas Safety Issue.

\subsubsection{Flammable Gas Safety Issue Resolution}

The report documenting all the data and analyses required to close the Flammable Gas Safety Issue was submitted in July 2001 (Johnson et al. 2001). Resolution of the Safety Issue was based on showing that the tanks and tank farm operations were within an approved safety envelope, that waste conditions were properly analyzed and mitigated as necessary, and that loss of primary or secondary containment of waste could not occur.

The closure of all open flammable gas USQs satisfied the requirement that the tanks be operated within their approved Authorization Basis safety envelope. The report discussed the characterization and evaluation of SY-101 and the remediation of the tank such that it could return to normal service. Evaluations for the rest of the DSTs and SSTs were also summarized from prior reports (Hedengren et al. 2000, 2001). In response to this information, DOE Headquarters closed the Flammable Gas Safety Issue on August 17, 2001.

\subsection{Local and Global Waste Disturbances}

If waste that contains gas bubbles is disturbed, some gas will be released from the volume that is actually disrupted. Whether a disturbance is local or global depends on the size of the disturbance relative to the waste volume. For example, penetration of the waste by a 3-inchdiameter core drill string is a local disturbance, while mixer pump operation, which eventually disturbs the entire waste volume, is global. The history of observations of gas releases during local and global disturbances is summarized below.

\subsubsection{Gas Releases Observed During Local Disturbances}

A 1996 review of 77 waste-intrusive activities in 47 separate SSTs showed three probable releases of gas associated with the waste disturbance detected with headspace gas monitoring equipment. The study then examined gas releases from an additional 61 core-sampling events and four additional liquid observation well (LOW) installations. These measurements were made using a hand-held combustible gas meter at the riser level before the drill string was flushed and after the sample was removed. Only three samples showed a measurable rise in hydrogen concentration in SSTs (Hedengren et al. 2001).

Additionally, the database of reportable events was reviewed for the time since extensive monitoring controls were implemented on the tanks (about five years). The only instances

(a) Conway JT. 1999. Letter (no title) to B. Richardson, U.S. Department of Energy, November 15, 1999, Defense Nuclear Facilities Safety Board, Washington, D.C. 
identified in SSTs where monitored flammable gas levels were $>25 \%$ of the LFL were in sealed risers or other sealed equipment that was left in a condition where it penetrated the waste for an extended period of time. The gas levels were determined to have resulted from a buildup of gases that were not able to escape or to mix with headspace gases. There were no reports identified of $>25 \%$ of the LFL in dome or ex-tank regions during waste-intrusive events during this time. While the specific number of waste-intrusive events has not been calculated, it is estimated that the number could easily be in the many hundreds.

The record is similar for the DSTs. During the 1990s, most of the DSTs were sampled with full-length core samplers. The core sampling consisted of penetrating the waste surface crust with a water lance followed by inserting the $\sim 3$-inch-diameter core sampler slowly into the waste until the tank bottom was reached. At least two cores were taken in each sampling event. No significant gas release (i.e., a surface level drop of 0.1 inch or greater or measured hydrogen concentration greater than $1,000 \mathrm{ppm}$ hydrogen) was observed for any of these events. Video monitoring of some of the sample events did show bubbling around the lance or sampler shaft, but insufficient gas was released to be detectable even by the headspace gas monitors.

Each DST on the Flammable Gas Watch List was also investigated using the ball rheometer and the void fraction instrument (VFI). The ball rheometer is a 4-inch-diameter, 16-1b ball attached to a cable. The ball is lowered and raised through the waste at varying speeds. The VFI has a 3-ft arm that allows it to sweep an area 6-ft in diameter around its support shaft. The VFI is slowly lowered and raised through the waste taking measurements about every six inches. Each tank was examined through at least two risers. Measurable gas releases were observed only during one deployment of the ball rheometer and VFI in Tank AW-101 when the hydrogen concentration reached 4,600 ppm. The release did not affect operations. Because gas release activity in this tank was particularly active during this period, the small disturbance probably triggered an event that would have occurred spontaneously at about the same time (Hedengren et al. 2000).

\subsubsection{Gas Releases Observed During Global Waste Disturbances}

Global waste disturbances have been mainly connected with retrieval operations and have included saltwell pumping, mixing, transfers, dissolution, and sluicing. This section summarizes some of the activities and the associated gas releases that might indicate the potential behavior of similar future operations.

Saltwell pumping has been performed for many years in a large number of SSTs, and a considerable experience base has been accumulated for gas releases. Saltwell pumping drains supernatant and as much of the interstitial liquid as is practical with a jet pump installed in a central well. The process usually requires about a year to complete. The maximum hydrogen concentration that has been observed in these passively ventilated tanks during saltwell pumping is $7,200 \mathrm{ppm}$, less than $25 \%$ of the LFL but greater than the action level of $6,250 \mathrm{ppm}$. Typically, the headspace is monitored periodically and pumping halted if hydrogen levels approach the action level. The fraction of stored gas released during saltwell pumping has ranged from 30 to $50 \%$, implying that over $50 \%$ of the gas remains in the waste after pumping (Hedengren et al. 2001). 
Jet pump mixing of a tank with a large retained gas volume has been done only in SY-101. This tank was degassed by mixing the waste with a single 150-hp jet pump during October through December 1993 (Allemann et al. 1994). The headspace hydrogen concentration remained at less than 1,000 ppm throughout and exceeded $500 \mathrm{ppm}$ on only three occasions in the initial stages of mixing. Repeated mixer pump runs performed on an average of three times per week for 25 minutes created a relatively uniform slurry with 1 to $2 \mathrm{~m}$ of sediment settling out between pump runs. The more recent mixer pump tests in Tank AZ-101 were not representative of a significant degassing operation. Tank AZ-101 had a relatively thin sediment layer and low retained gas volume, and the mixer pump jets were placed just above the sediment.

While mixing in SY-101 prevented gas accumulation in the sediment layer, which had been producing large buoyant displacement gas release events (BDGREs), it caused or allowed the slow growth of a floating crust layer. This caused an accelerating level rise that began to challenge the waste level limits in 1998-1999. The 3-m-thick crust contained as much gas or more than was stored in the waste prior to mixing. The surface level rise issue in SY-101 was remediated by a large-volume dilution and transfer that dissolved the thick crust layer and most of the soluble sodium salts in the tank (Mahoney et al. 2000). Dissolution-induced gas releases were largest when the crust was dissolved in January 2000. The dissolution process lasted several days and raised the hydrogen concentration as high as 3,000 ppm at one point. The larger gas releases were apparently caused by a breakup of large sections of crust, which liberated large volumes of "bubble slurry" (slurry that is approximately 50\% gas) that had accumulated beneath it.

Sluicing was performed regularly in the $1950 \mathrm{~s}$, ' $60 \mathrm{~s}$, and ' $70 \mathrm{~s}$. The only recent example that was sufficiently instrumented to assess gas release was sluicing of sludge from $\mathrm{C}-106$ into AY-102 to resolve the high-heat safety issue. C-106 contained about $1.8 \mathrm{~m}$ of sludge containing a 2 to 5\% gas volume fraction (Stewart and Chen 1998). Sluicing was performed very gently in this tank because of concern for postulated "steam bumps" resulting from reducing the hydrostatic head on sludge near the local boiling point. Each sluicing batch removed several inches to a foot of sludge and resulted in an increase in headspace hydrogen concentration from a baseline of 20-30 ppm to 400-500 ppm. Hydrogen was also liberated in the receiver tank, AY-102, usually increasing to about 100 ppm during sluicing (Cuta et al. 2000).

It is apparent that, even though some of these global waste disturbances were relatively rapid and severe, the peak hydrogen concentrations remained far below the LFL. The hydrogen concentration was more dependent on the ventilation rate than on the waste disturbance. The foregoing history does not point to an undue or unique hazard potential for future globally disturbing retrieval operations.

\subsection{Current and Planned Operations in Hanford Tanks}

New retrieval methods being proposed include saltcake dissolution, supernatant decant, and others. In addition, other globally disturbing operations such as transfers and mixing have been performed historically that are being brought to the same level of analysis as the proposed operations now under scrutiny. This section briefly summarizes the nine globally waste- 
disturbing activities that will be covered by the revised Authorization Basis. Each is described and evaluated thoroughly in Section 3.

In addition to these authorized activities, other potential globally waste-disturbing activities are being proposed whose potential for gas release will eventually need to be evaluated. In parallel with the saltcake dissolution retrieval demonstration in Tank S-112, a mechanical retrieval system is being developed for a demonstration in Tank C-104 using a robotic crawler. A pulsed-jet hydraulic system will be used to retrieve the waste in Tank S-102. Other retrieval methods may be proposed as well. However, the associated global waste disturbances are not sufficiently well defined to be analyzed at this time. Finally, major earthquakes also represent a globally waste-disturbing activity that can induce large gas releases (Reid and Deibler 1997). However, these events cannot be planned or controlled as part of the Authorization Basis and are not considered in this report.

\subsubsection{Waste Removal}

Fluid waste (liquid or liquid-solid slurry) is removed from a DST by pumping. The pumping action itself is assumed not to cause a significant waste disturbance. Transferring waste out of a tank disturbs the waste globally by reducing the hydrostatic pressure in the sediment. The resulting expansion of retained gas bubbles can cause sections of the sediment layer to become buoyant and release a large fraction of its gas in a BDGRE. At the same time, the first BDGRE can also suspend sediment that increases the bulk supernatant density. This reduces the gas fraction that is required to make the sediment buoyant (the neutral buoyancy gas fraction) and can thereby induce secondary BDGREs. Waste removal includes the proposed supernatant decant operation, which has been analyzed in detail (Wells et al. 2002).

\subsubsection{Waste Addition}

Fluid waste (liquid waste or liquid-solid slurry) is transferred into a DST by pumping such that the actual introduction of waste causes only a local disturbance. Waste addition increases the hydrostatic pressure on the entire waste volume and potentially changes the density of the liquid layer. Increasing hydrostatic pressure, which compresses retained gas, or adding waste that is less dense than the existing supernatant, which increases the neutral buoyancy gas fraction, both act to prevent gas release. However, adding fluid that is denser than the supernatant reduces the sediment neutral buoyancy gas fraction, which can induce BDGREs. The first buoyant displacement can suspend additional sediment, further reducing the neutral buoyancy gas fraction to induce additional gas releases.

\subsubsection{Saltwell Pumping}

Saltwell pumping is designed to remove supernatant and drainable interstitial liquid from SSTs to reduce the potential for releases of waste into the soil if a tank leaks. It is accomplished with a jet pump installed in a centrally located saltwell screen. The global waste disturbance of saltwell pumping comprises the removal of liquid itself and the resulting decrease in hydrostatic pressure in the liquid. 
In saltcake, the liquid drains from the pores between the particles. This removes the buoyant force on solid particles and increases the lithostatic load in the waste column. As described in Section 2, this changes the retained gas bubble configuration to a form with different release behavior. Retained gas is released from the draining saltcake at a rate roughly proportional to the brine-drainage rate. ${ }^{(a)}$ Dissolved ammonia is also released from the moist, newly unsaturated waste by evaporation. In the history of saltwell pumping, monitored hydrogen concentrations have not exceeded 1 vol\% (Hedengren et al. 2001).

In contrast to saltcake, sludge is observed to drain by consolidation of the matrix rather than by flow of liquid through a fixed porous structure, as in coarser saltcake, and is thus always effectively saturated. This is evidenced by theory and experiment (Simmons 1996). Thus the only mechanism for gas release during saltwell pumping of sludge tanks might be bubble percolation resulting from decreased hydrostatic pressure. Little enhancement to ammonia evaporation would be expected. All references to gas release behavior during saltwell pumping in the balance of this report refer to saltcake.

\subsubsection{Saltcake Dissolution}

Saltcake dissolution is proposed as a method of waste retrieval from SSTs containing a large fraction of soluble waste (Estey et al. 2001). For SST retrieval, water is sprayed on the waste to dissolve the soluble salts, and the resulting brine is pumped out of the tank at about the same average rate that it is created. The global waste disturbance of saltcake dissolution is the destruction of the sediment microstructure by dissolution of solid particles. Any gas bubbles retained in the region of the waste in which soluble solids are dissolved are assumed to be released. This activity presently only applies to SSTs.

\subsubsection{Water Addition}

Additions of relatively small volumes of water are sometimes necessary to flush transfer lines, lance instruments into the waste, decontaminate hardware removed from the waste, or install retrieval systems. The global waste disturbance potentially occurs as an increase in hydrostatic pressure and by dissolution of soluble solids, which is the primary gas release mechanism.

\subsubsection{Mixer Pump Operation}

DOE plans to install mixer pumps as part of DST waste retrieval and staging to the vitrification plant. A mixer pump degassed the SY-101 waste in 1993, and mixer pumps were tested in AZ-101 in 2001. Mixer pump jets globally disturb the waste by hydraulic erosion and suspension of previously settled sediment. Gas release during mixer pump operation is mainly caused by disruption of the waste structure that retains the gas bubbles. However, a major secondary release mechanism is the increase in effective liquid density by suspension of solid

(a) Huckaby JL, LA Mahoney, and ER Siciliano. 2001. Waste Gas Releases Associated with Recent Saltwell Pumping of Single-Shell Tanks. TWS01.036. Pacific Northwest National Laboratory, Richland, WA. 
particles. This reduces the neutral buoyancy gas fraction, which may induce BDGREs. Wells et al. (2002) performed detailed analyses of these effects for four DSTs.

\subsubsection{Airlift Circulator Operation}

Airlift circulators (ALCs) were designed into the construction of the AY and AZ DST farms as well as in Tanks AN-107 and AW-102 and SSTs in several farms. ALCs mix the waste by introducing a stream of air bubbles into large cylindrical tubes that extend from near the tank bottom well up into the supernatant. The bubble stream reduces the average density inside the large tubes, causing a strong upward flow. As many as 20 ALCs may be installed in a tank. The global disturbance by ALC operation is similar to mixing except that the hydraulic action of a high-velocity jet is absent. The main potential for gas release lies in mobilizing the sediment around the circulator tubes. As with mixer pump operation, the suspension of solids by the ALCs could also induce BDGREs by reducing the neutral buoyancy gas fraction.

\subsubsection{Chemical Addition}

Concentrated sodium hydroxide and possibly sodium nitrite solutions need to be added to DSTs occasionally to keep the waste chemistry within corrosion control limits. Each addition is typically on the order of 50,000 gallons of solution with a specific gravity as high as 1.5 (Fort 2001). The global disturbance is a relatively small increase in the hydrostatic pressure, the same as a waste addition. However, the high density of the solution also increases the bulk density of the supernatant. The corresponding decrease in the neutral buoyancy gas fraction could induce a buoyant displacement in a tank that already experiences them.

\subsubsection{Natural Evaporation}

Evaporation of water from the waste is included for completeness. It occurs naturally in all tanks but is of potential concern only in tanks with dilute waste and a high heat loading. Evaporation has been insignificant in concentrated saltcake tanks. Evaporation of water increases the concentration of dissolved solids in the supernatant and raises its density. Carried to an extreme, the rising supernatant density could theoretically reduce the neutral buoyancy gas fraction sufficiently to allow BDGREs. However, the increasing concentration of the liquid eventually suppresses evaporation, so the process is self-limiting. 


\subsection{Physics of Gas Generation, Retention, and Release}

Gas is generated in the waste by radiolysis of water, thermal and radiolytic decomposition of organic complexants, and corrosion of the steel tank walls. The gases consist mainly of hydrogen, nitrous oxide, and nitrogen with small amounts of ammonia, methane, and other hydrocarbons that are found in the tank headspaces and stored as bubbles in the liquid and solid wastes (Johnson et al. 1997; Mahoney et al. 1999).

The gas generation rate is an important parameter in determining whether a tank can retain sufficient gas to make the sediment buoyant. The steady-state hydrogen generation rate must also be considered in assessing 1) whether a tank's ventilation rate is sufficient to keep the headspace below the lower flammability limit (LFL) or, 2) if active ventilation fails, the time available to repair the system before the LFL is reached. Gas generation theory and modeling are summarized in Section 2.1.

Gas molecules are generated in the liquid. Because the gases, except ammonia, are not very soluble in concentrated salt solutions, most of the gas comes out of solution as bubbles. Ammonia is very soluble and remains mostly in solution. Though dissolved ammonia can be released by evaporation from a stirred liquid surface, it is not very flammable and is more a toxicological than a flammability hazard. Bubbles containing flammable gases are the most important mode of gas retention and release concerning flammability. Bubbles are retained only in those regions of the sediment that are otherwise saturated with liquid. The configuration and amount of gas retained depends mainly on the properties of the sediment, as described in Section 2.2.

The mechanisms of gas release and the behavior of a specific tank depend on the waste type and configuration. Potentially hazardous spontaneous releases are possible only in tanks where a deep layer of liquid overlies a deep sediment layer. These events (BDGREs) occur when portions of the sediment accumulate enough gas to become buoyant with respect to the liquid above. Models have been developed to determine whether a specific tank will exhibit these releases and whether they are likely to be induced by waste disturbing activities. Gas release behavior and the models currently used to evaluate it are described in Section 2.3.

Finally, a methodology has been developed to classify tanks by "waste groups" based on the accumulated knowledge of gas generation, retention, and release behavior (Barker and Hedengren 2002). This methodology provides a sound quantitative basis with which to apply controls to tanks based on the actual flammability hazard they present. The waste group definitions and criteria are summarized in Section 2.4.

\subsection{Gas Generation}

Stock (2000) reviewed the work that has been done during the past decade at universities, national laboratories, and the Hanford Site to establish the chemical origins of the gases generated in Hanford waste. Section 2.1.1 briefly summarizes Stock's review. An empirical 
model was developed by $\mathrm{Hu}$ (2000) based on this theory and comparisons with field observations of background hydrogen release rates. This model is summarized in Section 2.1.2.

\subsubsection{Gas Generation Theory}

Hydrogen is formed in Hanford Site waste in three distinct ways: first, by the radiolysis of water; second, during the corrosion of the steel tank walls; third, during the cascade of radiolytic and chemical reactions involved in the decomposition of organic compounds in the waste.

Organic complexants, organic phosphate esters, and organic hydrocarbons were used at Hanford during separations and other operations. The complex series of degradation reactions of the original compounds has created a broad array of fragmented and oxidized organic compounds. These compounds are also degraded so that the wastes now contain hundreds of different organic compounds. Eventually, slow aging reactions will convert organic carbon into inorganic carbonate ions. A product of many of these reactions is gas, such as hydrogen and other gases found in the tank waste.

Only a small portion of the hydrogen that is formed in the waste is produced through direct radiolysis of the organic compounds because the concentrations (electron density) of the organic compounds are very low compared with water and inorganic salts. Rather, most of the organicderived hydrogen and other gases are formed in the reactions of the reactive organic intermediates in the later stages of the chemistry.

Methane and other volatile organic compounds are formed via the degradation of organic complexants, extractants, and solvents used in Hanford Site separation processes and in the waste tanks. Production rates and competing reactions (e.g., reactions that degrade the organics to nonvolatile species) are such that the volatile organic compounds are minor waste gases in all but a few passively ventilated SSTs and do not represent a flammability hazard in any tank.

Ammonia arises in part from the oxidation reactions of the nitrogen-containing complexants. The reaction sequences that degrade EDTA and HEDTA lead to the formation of a molecule with a primary amino group and eventually to ammonia. The radiochemical reactions of glycine, which is a common intermediate in the degradation of these complexants, provide ammonia as one of the reaction products. Ammonia also is formed by the hydrolysis of nitriles and amides that are produced during the oxidation reactions of other organic compounds in the waste.

Laboratory investigations indicate that organic compounds are intimately involved in the formation of nitrogen, nitrous oxide, and additional ammonia. Nitric oxide and nitrogen dioxide are important reagents in reaction cascades that lead to the oxidation of the organic compounds and simultaneously to the reduction of nitrate or nitrite ions. Nitrous oxide is also formed by reaction pathways that involve the nitrosyl anion. Studies have demonstrated that these gas generation rates are highly sensitive to temperature and depend on the concentrations of the radioactive isotopes, principally strontium and cesium; organic compounds; and inorganic reagents such as aluminate, nitrite, nitrate, and hydroxide ion. 


\subsubsection{Hydrogen Generation Model}

$\mathrm{Hu}$ (2000) developed a hydrogen generation rate model based on a large body of gas generation and tank waste characterization data. The rate equations, which are formulated as a function of physical and chemical properties of tank waste, are used to estimate the hydrogen generation rate of current waste content as well as newly mixed waste of known waste properties. The model uses a set of semi-empirical rate equations to simulate the hydrogen generation mechanism of thermal chemical reactions, radiolysis of water and organic components, and corrosion processes. Hu specified the total hydrogen generation rate (HGR) in units of hydrogen yield per kilogram total waste per day as a sum of the thermolysis, radiolysis and corrosion rates:

$$
\mathrm{HGR}=\mathrm{HGR}_{\mathrm{thm}}+\mathrm{HGR}_{\mathrm{rad}}+\mathrm{HGR}_{\mathrm{corr}}
$$

where $\quad \mathrm{HGR}_{\mathrm{thm}}=\mathrm{a}_{\mathrm{thm}} \cdot \mathrm{r}_{\mathrm{f}} \cdot[\mathrm{TOC}] \cdot[\mathrm{Al}]^{0.4} \cdot \mathrm{L}_{\mathrm{f}} \cdot \mathrm{e}^{-(\mathrm{Ethm} / \mathrm{RT})}$

$\mathrm{HGR}_{\mathrm{rad}}=\left(\mathrm{G}_{\mathrm{H} 2 \mathrm{O}}+\mathrm{G}_{\mathrm{ORG}} \cdot \mathrm{L}_{\mathrm{f}}\right) \cdot \mathrm{H}_{\text {load }} \cdot \mathrm{CF}_{1}$

$$
\mathrm{HGR}_{\text {corr }}=\mathrm{R}_{\text {corr }} \cdot \mathrm{A}_{\text {wetted }} / \mathrm{M}_{\text {tank }} \cdot \mathrm{CF}_{2}
$$

with $\quad \mathrm{G}_{\text {org }} \quad=\mathrm{a}_{\mathrm{rad}} \cdot \mathrm{e}^{(-\mathrm{Erad} / \mathrm{RT})} \cdot\left(\mathrm{r}_{\mathrm{f}} \cdot[\mathrm{TOC}]\right)$

$$
\mathrm{G}_{\mathrm{H} 2 \mathrm{O}}=0.45-0.56 \cdot\left[\mathrm{NO}_{3}{ }^{-}\right]^{1 / 3}-0.43 \cdot\left[\mathrm{NO}_{2}{ }^{-}\right]^{1 / 3}
$$

and

$$
\begin{aligned}
& \mathrm{E}_{\mathrm{thm}} \quad \text { is activation energy for thermal reaction }(89.3 \mathrm{~kJ} / \mathrm{mole}) \\
& \mathrm{E}_{\mathrm{rad}} \quad \text { is activation energy for radiolytic reaction }(44.3 \mathrm{~kJ} / \mathrm{mole}) \\
& \mathrm{a}_{\mathrm{thm}} \quad \text { is pre-exponential factor for thermal reaction }(2.76 \mathrm{E}+09 \mathrm{~mole} / \mathrm{kg} \text {-day) } \\
& a_{\text {rad }} \quad \text { is pre-exponential factor for radiolytic reaction }\left(2.49 \mathrm{E}+06 \mathrm{H}_{2} / 100 \mathrm{eV}\right) \\
& \mathrm{G}_{\mathrm{ORG}} \quad \text { is hydrogen yield per } 100 \mathrm{eV} \text { energy from organic radiolysis } \\
& \mathrm{G}_{\mathrm{H} 2 \mathrm{O}} \quad \text { is hydrogen yield per } 100 \mathrm{eV} \text { energy from water radiolysis } \\
& \left(\mathrm{G}_{\mathrm{H} 2 \mathrm{O}} \text { has a minimum value of } 0.005 \mathrm{H}_{2} / 100 \mathrm{eV}\right) \\
& r_{f} \quad \text { is total organic carbon (TOC) reactivity coefficient } \\
& \text { (average value is } 0.7 \text { for DSTs and } 0.4 \text { for SSTs) } \\
& \mathrm{R} \quad \text { is } 8.314 \mathrm{~J} / \mathrm{mole} / \mathrm{K} \text {, gas constant } \\
& \mathrm{R}_{\text {corr }} \quad \text { is corrosion coefficient } \\
& \text { [1.83E-08 for DSTs and 3.6E-08 for SSTs }\left(\mathrm{m}^{3} / \mathrm{min} / \mathrm{m}^{2}\right) \text { at } 25^{\circ} \mathrm{C} \text { ] } \\
& \text { [TOC] is TOC concentration in the liquid waste ( } \mathrm{wt} \% \text { ) } \\
& \text { [Al] is aluminum concentration in the liquid waste }(\mathrm{wt} \%) \\
& {\left[\mathrm{NO}_{3}{ }^{-}\right] \text {is nitrate ion concentration in the liquid waste (moles/L) }} \\
& {\left[\mathrm{NO}_{2}{ }^{-}\right] \text {is nitrite ion concentration in the liquid waste (moles/L) }} \\
& \mathrm{H}_{\text {load }} \quad \text { is heat load }(\mathrm{W} / \mathrm{kg}) \\
& \mathrm{L}_{\mathrm{f}} \quad \text { is liquid weight fraction in the waste. } \\
& \mathrm{T} \quad \text { is temperature of the waste }(\mathrm{K}) \\
& \mathrm{A}_{\text {wetted }} \quad \text { is wetted surface area of the tank }\left(\mathrm{m}^{2}\right) \\
& \mathrm{M}_{\text {tank }} \quad \text { is total waste mass }(\mathrm{kg}) \text { in the tank } \\
& \mathrm{CF}_{1} \quad \text { is conversion factor to convert the units from }\left(\mathrm{H}_{2} / 100 \mathrm{eV}\right)(\mathrm{W} / \mathrm{kg}) \text { to } \\
& \text { (mole/kg-day) } \\
& \mathrm{CF}_{2} \text { is conversion factor to convert the units from } \mathrm{m}^{3} / \mathrm{kg} \text {-min to mole } / \mathrm{kg} \text {-day. }
\end{aligned}
$$


The analysis of all available gas generation data ( $\mathrm{Hu} 2000)$ shows that the temperaturedependent reaction, $\mathrm{HGR}_{\text {thm }}$, in Eq. (2.2) follows Arrhenius behavior, which can result from a multistep degradation of organic compounds initiated by radiolysis and followed by thermal reactions. The portion of hydrogen generated by radiolysis, $\mathrm{HGR}_{\mathrm{rad}}$, Eq. (2.3), depends on the radiation dose. The G-value for hydrogen generation from radiolysis of organic compounds, $\mathrm{G}_{\mathrm{org}}$, in Eq. (2.5) is also temperature dependent and follows Arrhenius behavior. The water radiolysis rate is temperature independent, with the $\mathrm{G}$-value, $\mathrm{G}_{\mathrm{H} 2 \mathrm{O}}$, in Eq. (2.6) reduced by scavenging by nitrate and nitrite ions.

The numerical values in the equations were established by the analysis of gas generation kinetic data from waste samples with the aid of tank field surveillance data and tank waste characterization data. The reactivity coefficient, $r_{f}$, was used to adjust for differences in reactivity of TOC among tanks. A detailed description of this model is given in $\mathrm{Hu}$ (2000).

$\mathrm{Hu}$ (2000) compared the predictions of the model with the observed hydrogen gas release rates in 28 tanks for which enough data were available to determine a release rate. Comparison of the calculated generation rates and the observed release rates for the 28 tanks indicated that the calculated generation rates generally were within a factor of 2 to 3 of the field observations. $\mathrm{Hu}(2000)$ also found that the calculated rates of gas generation by radiolysis and thermolysis in moles of hydrogen per kg of waste per day spanned a large range for these 28 tanks in accord with the variations in the chemical and physical properties of the wastes.

The calculated amounts of hydrogen generated because of corrosion are considerably more uniform and smaller. When the conditions for the thermolytic and the radiolytic generation of hydrogen are unfavorable, corrosion is the dominant source of hydrogen. The model indicates that this situation prevails in 14 of the 28 tanks and that these tanks generate from 10 to $90 \mathrm{~L} / \mathrm{d}$ of hydrogen. In contrast, the predicted rates of hydrogen generation for the 14 tanks for which radiolysis and thermolysis are dominant ranged from 100 to $930 \mathrm{~L} / \mathrm{d}$.

\subsection{Gas Bubble Retention Mechanisms}

The principal mechanisms of gas bubble retention can be grouped into three categories: bubbles retained by direct attachment to particles (e.g., armored bubbles, attached bubbles, agglomerates, etc.), bubbles trapped between particles by capillary forces, and bubbles held within the waste by its strength. In sediment layers, bubble retention is dominated by waste strength and capillary forces, though it may be limited by the bulk buoyancy of the sediment. Pore-filling bubbles are held in the interstitial spaces or pores between solid particles by capillary forces. Bubbles can also push or displace the solid particles apart to form more or less round bubbles that are trapped by the strength of the surrounding liquid-solid matrix.

Gas release occurs when the bubble retention mechanisms fail. Pore-filling bubbles are not a flammability concern because they move slowly through the porous media. Individual particledisplacing bubbles containing enough gas to present a flammability concern cannot exist, but small particle-displacing bubbles can disengage from the waste relatively rapidly and produce measurable gas releases in the aggregate. 
This section explains the differences and limitations of the two major types of bubbles. Gas release behavior is discussed in Section 2.3.

\subsubsection{Bubbles Retained by Capillary Forces}

Bubbles can fill the interstitial spaces or pores between solid particles when the lithostatic load is sufficient to hold the particles in contact against the force of the bubble's internal pressure that acts to push them apart. The bubble pressure is controlled by capillary force in the throats between pores - the narrower the throat, the higher the pressure. This retention mechanism requires either relatively large particles whose larger pore throats reduce the internal bubble pressure or a deep waste column that increases the lithostatic load - or both. These bubbles assume an irregular, dendritic shape conforming to the passages between the particles. When the bubble's internal pressure is sufficient to overcome the capillary forces, it may still be restrained by the yield strength of the waste, as described in Section 2.2.2.

Whether a bubble is held by yield strength or capillary force depends on a Bond number criterion developed by Gauglitz et al. (1994, 1995, 1996). This dimensionless group is the sum of two parameters: a ratio of gravitational force to surface tension force and a ratio of waste strength force to surface tension force. If the Bond number exceeds unity, a bubble exists in the pore-filling configuration held by capillary forces between particles. The Bond number is expressed as

$$
\mathrm{N}_{\mathrm{Bo}}=\frac{\Delta \rho g \mathrm{H}_{\mathrm{S}} \mathrm{D}_{\mathrm{p}}}{4 \sigma}+\frac{\tau_{\mathrm{y}} \mathrm{D}_{\mathrm{p}}}{4 \sigma} \mathrm{A}
$$

where

$\mathrm{H}_{\mathrm{S}}=$ the height of the lithostatic column above the bubble

$\mathrm{D}_{\mathrm{P}}=$ the mean pore throat diameter through which a bubble must pass to escape retention; assumed to be represented by the particle diameter

$\Delta \rho=$ the difference between solid particle and liquid density

$\sigma=$ the surface tension

$\tau_{\mathrm{y}}=$ the bulk sediment yield stress

$\mathrm{A}=$ parameter related to how the yield stress resists bubble expansion; it was estimated at 2.8 by Gauglitz et al. (1995)

$\mathrm{g}=$ gravitational constant.

The upper limit on the vertical size of a pore-filling bubble can be derived from the balance of capillary forces and the hydrostatic pressure difference between the top and bottom of the bubble (Stewart et al. 1996). As the bubble grows, the radii of curvature of the liquid-gas interfaces in the pore throats continually adjust in response to the uniform and increasing gas pressure inside the bubble. The bubble internal pressure eventually exceeds the restraining force of surface tension and liquid hydrostatic head in throats on the upper surface of the bubble. This allows gas to push out of the top of the bubble, reducing the pressure to allow liquid to flow into the bottom of the bubble. The bubble thus moves upward until a new equilibrium is established. 
The gas volume fraction at which this motion occurs is called the percolation threshold. The maximum height, $\Delta \mathrm{h}$, that a pore-filling bubble can attain before percolating is expressed as

$$
\Delta \mathrm{h}=4 \sigma / \rho_{\mathrm{L}} \mathrm{gD}
$$

where $\rho_{\mathrm{L}}$ is the liquid density.

Pore-filling bubbles, though they may represent a considerable gas volume, are not a flammability concern because there is no mechanism for large numbers of pore-filling bubbles to be released rapidly. For gas in a pore-filling bubble to move, liquid must be displaced from the pores the bubble enters and flow into the pores the bubble vacates. Flow of liquid through the porous media of Hanford waste is generally a very slow process. Also, before a pore-filling bubble can be released into a tank's headspace, it must rise into a decreasing lithostatic load and eventually become a particle-displacing bubble, whose characteristics are described below.

\subsubsection{Gas Retained by Waste Strength}

The shape of a particle-displacing bubble held by the strength of the waste (as determined by the Bond number in Eq. 2.7) is determined by the relative effects of surface tension and waste strength. With relatively weak waste or small bubbles, surface tension pulls bubbles into an approximately spherical shape. If the effect of waste strength is greater than surface tension force, the bubble grows into the weakest area of the waste surrounding it and assumes an irregular, dendritic shape. Particle-displacing bubbles that are not dendritic are called "round" bubbles, even though they may be ellipsoidal or similarly distorted from a truly spherical shape. A criterion for bubble shape is derived from simple scaling of the relative importance of strength

to surface tension. The maximum diameter of round bubbles before they begin to assume a dendritic shape is given by

$$
\mathrm{D}_{\mathrm{b}}<\frac{\sigma}{\tau_{\mathrm{y}}}
$$

The ability of the material to resist the bubble's buoyancy limits the size of the bubble. A particle-displacing bubble can grow vertically only until the buoyant force exerted by the bubble exceeds the ability of the waste to hold it in place. A criterion for incipient motion of a solid sphere immersed in a Bingham fluid can be derived in terms of a critical-gravity yield number, $\mathrm{Y}_{\mathrm{G}}$. The resulting limiting diameter for upward motion of a bubble is expressed as

$$
\mathrm{D}_{\mathrm{b}}<\frac{\tau_{\mathrm{y}}}{\rho_{\mathrm{S}} \mathrm{gY} \mathrm{G}_{\mathrm{G}}}
$$

where $\rho_{\mathrm{S}}$ is the bulk density. The number $\mathrm{Y}_{\mathrm{G}}$ is estimated at 0.2 for use with typical yield strengths measured in Hanford tank waste. Using typical waste properties, Eq. (2.9) and (2.10) predict that the maximum diameter of a round bubble is about 0.5 to $1.0 \mathrm{~cm}$. This size is 
consistent with observations in waste and simulants (Rassat et al. 1997, 1998, Gauglitz et al. 1996). The important conclusion of this analysis is that a very large round bubble that could contain a hazardous amount of gas simply cannot exist.

Eq. (2.10) also gives the limiting height of dendritic particle-displacing bubbles. When they grow to the limiting size, the increased hydrostatic pressure difference pinches off the bottom of the bubble and pushes the top of the bubble upward in a manner similar to percolation of porefilling bubbles. However, motion of particle-displacing bubbles is much faster because it does not require liquid flow through a porous medium. If the waste is moderately strong, the bubbles do not collapse completely, and dendritic bubble networks can form to provide continuous gas release (Gauglitz et al. 1996).

\subsection{Gas Release Mechanisms and Behavior}

The mechanisms of gas release and the behavior of a specific tank depend on the waste type and configuration. The three main Hanford tank waste types are liquid (no or little solids present), sludge (insoluble solids) and saltcake (solids precipitated from saturated liquid on cooling). Liquid, by definition, is a dilute or concentrated solution of dissolved solids, water, and a small fraction of soluble organics that contains at most a small fraction of suspended solid particles. Sludge consists of fine insoluble particles that are mostly metal oxides and hydroxides. Saltcake forms by the settling of sodium and aluminum salts precipitated from a cooling saturated solution. Solid particles in saltcake are generally larger than those in sludge. Some tanks contain a mixture of sludge and saltcake that can be considered a "mixed" waste type. However, the mixed waste appears to behave like saltcake in most cases.

Waste configuration is the arrangement of waste types in layers within a tank. The waste types are distributed within the tanks in only a few different ways, depending mainly on the amount of liquid. In order of decreasing amount of liquid, the main waste configurations are 1) "liquid," containing almost entirely the liquid waste type; 2) "liquid-over-solid," where a relatively deep layer of supernatant liquid overlies an equally deep layer of liquid-saturated sediment; 3) "wet solids," where the sediment is saturated with liquid but there is little or no supernatant; and 4) "pumped," where interstitial liquid has been removed by saltwell pumping such that its level is well below the waste surface.

Two other waste configurations are also of interest: "crust," a solid-over-liquid arrangement consisting of a floating layer of gas-bearing solids, and "mixed slurry," in which most of the solid particles and small gas bubbles are kept in suspension mechanically. A crust exists only with the more concentrated saltcake wastes in a few DSTs as a modification of the liquid-oversolid configuration. The SSTs A-101 and AX-101 are unique in that, based on core sample evidence, the entire mass of undissolved solids in the tank floats on a very dense liquid layer. However, this cannot be treated as a true "crust." Mixed slurry has existed only in SY-101 from 1993 to 2000, when the mixer pump was operating. The characteristics of gas retention and release in each of the four main waste configurations, liquid, liquid-over-solid, wet solids, and pumped, are discussed in more detail below. 


\subsubsection{Liquid Waste Configuration}

Only a few of the DSTs currently contain essentially all liquid. In these tanks, gas generated in solution by radiolysis nucleates to form small bubbles on suspended microparticles or on the tank walls. Once these bubble-particles grow large enough to become buoyant, they rise immediately to the surface and release gas into the headspace to be removed by ventilation. This continuous background release mechanism makes retention of gas bubbles physically impossible in this configuration.

\subsubsection{Liquid-over-Solid Configuration}

Except for two of the DSTs with thick crust layers, gas is retained mainly in the sediment. The liquid-over-solid waste configuration in saltcake DSTs is subject to BDGREs that are the largest and fastest releases known to occur in Hanford tanks and the only releases that are known to have created flammable conditions in a tank's headspace. Before the mixer pump was installed in SY-101, its buoyant displacements typically released over 5,000 scf of gas over a period of 10 to 20 minutes three times per year. At least two of these releases exceeded $10,000 \mathrm{scf}$, enough to make the tank headspace just flammable. Five other DSTs exhibit BDGREs that are much smaller, slower, and less frequent. None have reached flammability, and only two releases in AN-105 have exceeded $25 \%$ of the LFL.

The yield stress in the sediment of these five DSTs increases roughly linearly from zero at the upper surface to 200-300 $\mathrm{Pa}$ at the bottom (Hedengren et al. 2000). This strength is sufficient to hold bubbles up to about a centimeter in diameter. The particle size is relatively small so that Eq. (2.7) predicts only particle-displacing bubbles. The material strength is also sufficiently low that the bubbles remain approximately round, according to Eq. (2.8). Measurements made by the void fraction instrument (VFI) and retained gas sampler (RGS) show that gas volume fraction in the sediment layer increases with depth from near zero at the top. Two distinct gas fraction profiles were observed: one approximately parabolic, with a peak at about the midpoint of the sediment layer; and another roughly linear, with the maximum at the bottom. The peak gas fraction ranges from 0.1 to 0.16 with averages from 0.04 to 0.11 (Hedengren et al. 2000).

BDGREs occur in saltcake tanks with a deep layer of supernatant liquid when a portion, or "gob," of the sediment accumulates enough gas to become buoyant with respect to the liquid above it, breaks away, and rises through the liquid. The stored gas bubbles expand as the gob rises, failing the surrounding material so a portion of the gas can escape into the headspace. After releasing gas until it is no longer buoyant, the gob sinks back to rejoin the sediment. This process can be described in terms of several criteria based on the waste properties and configuration (Meyer and Stewart 2001).

\subsubsection{Buoyancy Ratio Criterion}

Gas accumulation in the sediment is a balance between gas generation and steady background gas release. The background release is assumed to be a slow migration of bubbles that qualitatively obeys the form of Stokes' Law. Consistent with in situ measurements, the waste 
viscosity (determining bubble rise velocity) is assumed to increase linearly with waste depth from zero at the top of the layer. The gas volume fraction (also called void fraction) profile can be determined from the equations for conservation of bubble mass and number (Meyer and Stewart 2001). Assuming a uniform bubble nucleation rate and gas generation rate, the solution is a parabolic distribution given by

$$
\alpha(\eta)=\frac{C_{B}}{\rho_{S}}\left(\frac{G T}{P_{S}}\right)^{\frac{1}{3}} H_{S}^{2} \eta(1-\eta)
$$

where $\rho_{\mathrm{S}}$ is the degassed sediment density (measured), $\mathrm{T}$ is the average sediment temperature (measured), $\mathrm{P}_{\mathrm{S}}$ is the average pressure in the retained gas (calculated from measured waste physical properties), $G$ is the volumetric gas generation rate (calculated from measured retained and released gas concentrations and waste chemical and physical properties), $\mathrm{H}_{\mathrm{S}}$ is the depth of the sediment layer (measured), and $\eta=\mathrm{z} / \mathrm{H}_{\mathrm{S}}$, where $\mathrm{z}$ is the distance from the tank bottom. If the average gas fraction in the sediment layer predicted by Eq. (2.11) exceeds the neutral buoyancy gas fraction, a BDGRE will occur. The coefficient $C_{B}$ is adjusted so that the tanks currently exhibiting buoyant displacements are predicted to exceed neutral buoyancy. The neutral buoyancy gas fraction is defined by the waste density as follows:

$$
\alpha_{\mathrm{NB}}=1-\frac{\rho_{\mathrm{L}}}{\rho_{\mathrm{S}}}
$$

where $\rho_{\mathrm{L}}$ is the liquid density (measured). The criterion of Eq. (2.11) and (2.12) is usually expressed as the buoyancy ratio, the average gas fraction obtained by integrating Eq. (2.11) divided by the neutral buoyancy gas fraction, Eq. (2.12). The buoyancy ratio is expressed by

$$
\mathrm{BR}=\frac{\alpha_{\text {avg }}}{\alpha_{\mathrm{NB}}}=\frac{\mathrm{C}_{\mathrm{B}}}{\rho_{\mathrm{S}}-\rho_{\mathrm{L}}}\left(\frac{\mathrm{GT}}{\mathrm{P}_{\mathrm{S}}}\right)^{1 / 3} \mathrm{H}_{\mathrm{S}}^{2}
$$

If the buoyancy ratio is unity or greater, BDGREs can be expected.

\subsubsection{Energy Ratio Criterion}

The process of gas release from a gob undergoing buoyant displacement requires that sufficient energy be released to disrupt the waste surrounding the bubbles to allow them to escape as the gob reaches the waste surface. The amount of energy available is directly proportional to the depth of the supernatant through which the gob rises. The amount of work required to yield the gob is directly proportional to the yield stress of the material. In addition, a large fraction of the buoyant energy is dissipated in other processes so the required energy is much greater than that which would just yield the waste. 
Stewart et al. (1996) developed an energy model to account for this. The ratio between the buoyant energy, $\mathrm{E}_{\mathrm{b}}$, and the energy required to yield the gas-bearing gob participating in the buoyant displacement, $\mathrm{E}_{\mathrm{y}}$, may be expressed as

$$
\frac{\mathrm{E}_{\mathrm{b}}}{\mathrm{E}_{\mathrm{y}}}=\frac{\alpha_{\mathrm{C}} \rho_{\mathrm{L}} \mathrm{gh}}{\left(1-\alpha_{\mathrm{C}}\right) \varepsilon_{\mathrm{y}} \tau_{\mathrm{y}}}\left\lfloor\left(1+\frac{1}{\gamma}\right) \ln (1+\gamma)-\mathrm{k}\right\rfloor
$$

where $\mathrm{h}$ is the distance from the top of the participating gob to the top of the liquid layer and $\varepsilon_{\mathrm{y}}$ is the strain at failure, which is taken to be unity. The parameters $\gamma, \mathrm{k}$, and $\alpha_{\mathrm{C}}$ are determined from

$$
\begin{aligned}
& \gamma=\frac{\rho_{\mathrm{L}} g h}{\mathrm{P}_{\mathrm{A}}} \\
& \mathrm{k}=\frac{\alpha_{\mathrm{NB}}\left(1-\alpha_{\mathrm{C}}\right)}{\alpha_{\mathrm{C}}\left(1-\alpha_{\mathrm{NB}}\right)} \\
& \alpha_{\mathrm{C}}=\alpha_{\mathrm{NB}}+\frac{\beta \tau_{\mathrm{y}}}{\rho_{\mathrm{S}} \mathrm{gH}_{\mathrm{S}}}
\end{aligned}
$$

where $\beta$ is ratio of the yield stress in tension to the yield stress in pure shear, which is taken to be unity. For weak waste with $\tau_{\mathrm{y}}$ much less than $\rho_{\mathrm{S}} \mathrm{gH} \mathrm{H}_{\mathrm{S}}, \alpha_{\mathrm{C}}=\alpha_{\mathrm{NB}}$, and Eq. (2.14) can be written as

$$
\frac{E_{b}}{E_{y}}=\frac{\left(\rho_{S}-\rho_{L}\right) g h}{\varepsilon_{y} \tau_{y}}\left[\left(1+\frac{1}{\gamma}\right) \ln (1+\gamma)-1\right]
$$

Based on experimental observations and tank behavior, some gas can be released when the energy ratio exceeds 3 , and releases of a large fraction of the stored gas can occur above energy ratios of 5 .

\subsubsection{Other BDGRE Criteria}

Several empirical relationships have also been used that correlate groupings of physical parameters with BDGRE behavior. The single waste property found to most closely correlate with BDGRE behavior was tank average specific gravity or density. ${ }^{\text {(a) }}$ A criterion was established for waste transfers (Fowler 1995) based on the weighted mean specific gravity of transferred waste and the waste in the receiver tank. If the average specific gravity exceeded 1.41, BDGRE behavior was considered possible. This criterion is not used in the revised tank waste group classification because it predicts that several tanks should exhibit BDGREs that do not.

(a) Reynolds DA. 1994. Evaluation of Specific Gravity versus Gas Retention. Internal memo 7E31094-024, Westinghouse Hanford Company, Richland, Washington. 
Estey and Guthrie (1996) found that the product of sediment depth and supernatant specific gravity gave a clearer separation than the average specific gravity between tanks that exhibited BDGREs and those that did not. If the product of sediment depth (in inches) and supernatant specific gravity exceeded 150 inches, BDGREs were considered possible. This criterion is not used in classifying waste because more recent data indicate one tank as having BDGREs that does not and fails to predict BDGREs in another tank that does.

\subsubsection{Wet Sediment Configuration}

Most of the tanks other than DSTs that retain a sizeable volume of gas consist entirely of sediment that is saturated with liquid but little or no supernatant. For the generally larger particles of saltcake waste, Eq. (2.7) states that the upper several meters will retain individual round bubbles while the bottom few meters will contain pore-filling bubbles due to the high lithostatic load. With the generally smaller particles of sludge, retained gas bubbles will exist as particle-displacing bubbles at any depth. The wet sediment tanks with saltcake waste type have the highest gas fraction and deepest waste, so they account for most of the total retained gas volume.

Unlike the liquid-over-sediment waste configuration, the gas retention characteristics of sludge and saltcake in wet sediment tanks appear to be quite different. The median gas fraction for sludge tanks is only 0.01 but is 0.12 for all saltcake tanks. An average gas fraction exceeding 0.25 has been measured in a saltcake tank; the sludge tanks only reach 0.12 . A large group of the sludge tanks exhibit a barely detectable gas fraction (Barker et al. 1999). This difference likely results from the generally lower waste depth, lower gas generation rate per unit volume, and smaller particle size of the sludge.

The characteristics of nonbuoyant displacement gas releases from the wet sediment configuration depend to a great degree on how the gas is stored. For example, pore-filling bubbles require liquid to flow through the porous media in order to migrate. This makes gas release a very slow process. Gas release from particle-displacing bubbles may be somewhat faster but is believed to be limited to a small region of a tank. This is confirmed by headspace gas monitoring data that show these tanks typically releasing on the order of 10 to $100 \mathrm{scf}$ of gas over a period of several days. These releases tend to occur when the barometric pressure falls rapidly during storm passage in the late fall and early spring.

\subsubsection{Pumped Configuration}

In pumped tanks containing saltcake, drainable liquid has been removed and the interstitial liquid level is usually well below the waste level. The portion of the waste above the liquid level exerts a disproportionately high lithostatic load on the waste below. Applying Eq. (2.7) with the density difference set equal to the solid density, only one meter of unsaturated saltcake above the liquid level is required to force the entire column into the pore-filling bubble configuration.

In pumped sludge tanks, liquid removal results in consolidation of the liquid-solid matrix rather than draining of a fixed porous structure (Simmons 1996). Thus, pumping does not create an unsaturated overburden in sludge like it does in saltcake, and the lithostatic load does not 
change very much. Even with an unsaturated layer, Eq. (2.7) indicates that the smaller sludge particles prevent formation of pore-filling bubbles. Trapped gas bubbles remain in a particledisplacing configuration in any case and tend not to release during pumping.

Gas fraction information for pumped tanks is available for some sludge tanks (Barker et al. 1999). Only one pumped saltcake tank, BY-109, has gas fraction data (Mahoney 2000). Note that BY-109, while predominantly saltcake (253 kgal), does contain $24 \mathrm{kgal}$ of sludge (Hanlon 2002). The limited data show there is essentially no reduction in gas fraction after pumping a sludge tank consistent with the theory above. The mean gas fraction below the interstitial liquid level in BY-109 measured with the retained gas sampler was 0.09, the lowest of all tanks measured with the RGS.

Gas release behavior during and after saltwell pumping of saltcake tanks is discussed in detail in Section 3.3.2. There is no known mechanism for large spontaneous gas release from a pumped tank.

\subsection{Revised Waste Classification}

As discussed above, release of retained gas depends on the form and nature of the waste as well as the nature of any waste-disturbing activities. To develop and implement cost-effective flammable gas control strategies, tanks are grouped according to waste form and gas release behavior.

\subsubsection{Waste Group Definition}

Three "waste groups" are defined based on the understanding of gas retention and release behavior to identify the tanks that pose a flammable gas hazard and to differentiate tanks that are susceptible to BDGREs from those that are not (Barker and Hedengren 2002). The waste group classifications for specific tanks subsequently presented in this document are subject to change and should not be referenced as the true waste group classifications. Actual waste group classifications are presented in Barker and Hedengren (2002). The following is a summary of the waste group classifications:

- Waste Group A: Includes tanks that have the propensity for spontaneous BDGREs and have sufficient retained gas to exceed the LFL if all of it is released suddenly. BDGREs may also be induced in these tanks by large waste disturbances that suspend sediment in the supernatant or otherwise increase its density.

- Waste Group B: Includes tanks that do not exhibit spontaneous BDGREs but have sufficient retained gas to exceed the LFL if all of it were released suddenly. Given the level of retained gas, significant gas releases potentially can be induced by large disturbances in the sediment.

- Waste Group C: Includes all DSTs and SSTs that do not have sufficient retained gas to achieve the LFL if all of the retained gas were released suddenly, regardless of whether they exhibit spontaneous BDGREs. 


\subsubsection{Criteria for Waste Group Classification}

The three criteria specified in Barker and Hedengren (2002) are used to place the tanks in the appropriate waste group. The first is a screening that evaluates the potential flammable gas hazard. Tanks with insufficient retained gas in the sediment layer to cause the tank headspace to become flammable if all of it were released at once are considered nonhazardous and are placed in Waste Group C. The sediment gas volume is the product of the waste volume calculated from the wet sediment depth and the average retained gas volume fraction. The gas volume fraction may be determined from measurements, assigned a conservative bounding value, or limited to the neutral buoyancy gas fraction for tanks with liquid-over-sediment waste configuration.

If a tank is shown to retain sufficient gas to make the headspace flammable, it is assigned to Waste Group A if it has the propensity for spontaneous BDGREs or to Waste Group B if it does not. The presence of a sufficient supernatant liquid layer is the first requirement for BDGREs. This requirement is evaluated by calculating the "energy ratio" defined by Eq. (2.14). Tanks with an energy ratio below the threshold value of 3 cannot release gas in a BDGRE and are assigned to Waste Group B. For typical tank conditions, this entails a supernatant depth of at least 1 to $2 \mathrm{~m}$, which exists only in the DSTs.

Tanks exceeding the energy threshold are further evaluated to determine whether buoyancy can be achieved. The buoyancy ratio, Eq. (2.13), is used for this evaluation. If the buoyancy ratio exceeds unity, BDGRE behavior is indicated and the tank is assigned to Waste Group A. 


\subsection{Gas Releases Induced by Global Waste Disturbances}

A global waste disturbance is one that affects all or a large fraction of the total waste volume. Global disturbances can range from a change in waste volume, with coincident change in hydrostatic pressure, to a complete redistribution of the waste mass as in mixer pump operation. The objective of this section is to evaluate the impacts of authorized globally waste-disturbing activities based on the dominant gas release mechanisms and tank waste group assignment described in Section 2.4. The need for gas monitoring, the potential long-term changes in waste group assignment, and increases in the steady-state hydrogen generation after the activity is completed are also included in the evaluation.

The authorized globally waste-disturbing activities covered in this section are summarized in Table 3.1. Generally, the activities are specific to either DSTs or SSTs based on the context of the operation. For example, saltwell pumping is treated separately from waste removal because saltwell pumping is performed only in SSTs. Similar nuances are explained in the detailed discussion below. Besides the waste disturbance itself, any activity that adds material to the tank also decreases the headspace, which exacerbates the consequences of gas releases. Except for natural evaporation, global waste disturbances associated with natural phenomena are not included because they are either of low probability (e.g., severe earthquakes) or low consequence (Johnson et al. 2001).

Table 3.1. Summary of Globally Waste-Disturbing Activities

\begin{tabular}{||l|l|l||}
\hline \multicolumn{1}{|c|}{$\begin{array}{c}\text { Activity } \\
\text { (tank type) }\end{array}$} & \multicolumn{1}{|c|}{ Description } & \multicolumn{1}{c|}{ Waste Disturbance } \\
\hline $\begin{array}{l}\text { Waste removal } \\
\text { (DST) }\end{array}$ & $\begin{array}{l}\text { Liquid or slurry pumped } \\
\text { out }\end{array}$ & $\begin{array}{l}\text { Reduced hydrostatic pressure and supernatant } \\
\text { depth }\end{array}$ \\
\hline $\begin{array}{l}\text { Waste addition } \\
\text { (DST) }\end{array}$ & $\begin{array}{l}\text { Liquid or slurry } \\
\text { transferred in }\end{array}$ & $\begin{array}{l}\text { Change in supernatant density, increased } \\
\text { supernatant and sediment depth and hydrostatic } \\
\text { pressure }\end{array}$ \\
\hline $\begin{array}{l}\text { Saltwell pumping } \\
\text { (SST) }\end{array}$ & $\begin{array}{l}\text { Supernatant and interstitial } \\
\text { liquid pumped out }\end{array}$ & $\begin{array}{l}\text { Interstitial liquid drainage, increased lithostatic } \\
\text { load, decreased wet sediment depth }\end{array}$ \\
\hline $\begin{array}{l}\text { Saltcake dissolution } \\
\text { (SST) }\end{array}$ & $\begin{array}{l}\text { Dissolution of soluble } \\
\text { solids by water addition } \\
\text { with brine removal }\end{array}$ & $\begin{array}{l}\text { Destruction of sediment structure, waste } \\
\text { volume reduction, decrease in lithostatic load }\end{array}$ \\
\hline $\begin{array}{l}\text { Water addition } \\
\text { (both) }\end{array}$ & $\begin{array}{l}\text { Water added without brine } \\
\text { removal }\end{array}$ & $\begin{array}{l}\text { Solids dissolution, increased hydrostatic head, } \\
\text { decreased supernatant density }\end{array}$ \\
\hline $\begin{array}{l}\text { Mixer pump } \\
\text { operation (DST) }\end{array}$ & $\begin{array}{l}\text { High-energy jet mobilizes } \\
\text { sediment }\end{array}$ & $\begin{array}{l}\text { Destruction of sediment structure, solids } \\
\text { suspended in supernatant }\end{array}$ \\
\hline $\begin{array}{l}\text { Airlift circulator } \\
\text { operation (DST) }\end{array}$ & $\begin{array}{l}\text { Low-energy circulation } \\
\text { mobilizes sediment }\end{array}$ & $\begin{array}{l}\text { Destruction of sediment structure, solids } \\
\text { suspended in supernatant }\end{array}$ \\
\hline $\begin{array}{l}\text { Chemical addition } \\
\text { (DST) }\end{array}$ & $\begin{array}{l}\text { High-density liquid added } \\
\text { to supernatant }\end{array}$ & $\begin{array}{l}\text { Increased hydrostatic pressure, supernatant } \\
\text { depth, and density }\end{array}$ \\
\hline $\begin{array}{l}\text { Natural evaporation } \\
\text { (DST) }\end{array}$ & Water loss to atmosphere & $\begin{array}{l}\text { Increased supernatant density, decreased } \\
\text { supernatant depth }\end{array}$ \\
\hline \hline
\end{tabular}




\subsection{Waste Removal}

Fluid waste is transferred out of a tank by pumping. The pumping process itself is considered a local disturbance whether the inlet is located in the supernatant or in the sediment. The fluid may all be liquid if the transfer pump inlet is located in the supernatant or a slurry if the inlet is in the sediment or the waste is mixed before or during transfer. However, in the latter case, the primary waste disturbance is the mixing operation, which is covered in Section 3.6. Essentially all of the retained gas would have been released by mixing, nullifying the potential consequences of waste removal. Removal of waste from a tank causes a global disturbance by reducing the hydrostatic pressure on the entire waste volume. The waste configuration is also altered by reducing the depth of supernatant, which also increases the tank headspace.

Waste removal is specific to DSTs. It includes the proposed supernatant decant operation, which was analyzed in detail by Wells et al. (2002) and whose results are summarized in Section 3.1.2. Waste removal from SSTs is considered under saltwell pumping in Section 3.3 or saltcake dissolution in Section 3.4.

\subsubsection{Mechanisms for Gas Releases During Waste Removal}

The primary mechanism of gas release from this activity is the reduction in hydrostatic pressure, as explained below. The resulting expansion of retained gas bubbles can cause a sediment layer to become buoyant or allow the expanded bubbles to disengage from the sediment. If a buoyant displacement occurs, the suspended sediment increases the supernatant bulk density, reducing the neutral buoyancy gas fraction and potentially inducing secondary BDGREs. The increased density also increases the hydrostatic pressure, which decreases the gas fraction and reduces the potential for a BDGRE. However, this effect is much less than that of buoyancy. If the entire sediment layer in AN-105 were suspended in the supernatant, for example, the neutral buoyancy gas fraction would decrease almost $60 \%$, from 0.095 to 0.054 , while the gas volume fraction of a gob initially just at neutral buoyancy would only decrease $2 \%$, from 0.095 to 0.093 .

Peak hydrogen concentrations resulting from BDGREs induced by waste removal are highest if supernatant is removed rather than sediment. If sediment were removed, the rate of hydrostatic pressure reduction on the gas in the undisturbed portion of the sediment layer would be slower. This would tend to induce BDGREs later into a larger headspace. The local disturbance of sediment pumping would also release some of the retained gas that would otherwise have been released in BDGREs.

Waste removal from a Group A tank ${ }^{(a)}$ will induce BDGREs of a magnitude similar to its historical spontaneous releases. A BDGRE can theoretically also be induced by waste removal in a Group B tank if the retained gas volume fraction is high enough to achieve buoyancy by the hydrostatic pressure reduction. The potential for an induced BDGRE in a Group B tank can be

(a) As described in Section 2.4.1, Waste Group A and B tanks store sufficient gas to make the headspace flammable if all of it were suddenly released; Waste Group C tanks do not. Waste Group A tanks exhibit spontaneous BDGREs, while tanks in Waste Group B do not. 
evaluated prior to waste removal as follows: The change in sediment retained gas volume fraction, $\alpha$, with the average sediment gas pressure, $P_{S}$, is given by (Wells et al. 2002)

$$
\frac{\mathrm{d} \alpha}{\mathrm{dP}_{\mathrm{S}}}=-\frac{\alpha(1-\alpha)}{\mathrm{P}_{\mathrm{S}}}
$$

Integrating Eq. (3.1.1) from initial state 0 to the final state 1 following waste removal yields

$$
\alpha_{1}=\left[\frac{\mathrm{P}_{1}}{\mathrm{P}_{0}}\left(\frac{1-\alpha_{0}}{\alpha_{0}}\right)+1\right]^{-1}
$$

We want to know the pressure reduction necessary to raise the initial gas fraction to the neutral buoyancy value, $\alpha_{\mathrm{NB}}$, defined by Eq. (2.12). Substituting $\alpha_{\mathrm{NB}}$ for $\alpha_{1}$ and solving for the pressure change gives

$$
\frac{\Delta \mathrm{P}}{\mathrm{P}_{0}}=-\frac{\alpha_{\mathrm{NB}}-\alpha_{0}}{\left(1-\alpha_{0}\right) \alpha_{\mathrm{NB}}}
$$

The initial average sediment gas pressure can be approximated by the hydrostatic pressure at the midpoint of the sediment:

$$
\mathrm{P}_{0}=\mathrm{P}_{\mathrm{A}}+\mathrm{g}\left[\rho_{\mathrm{L}}\left(\mathrm{H}_{\mathrm{L} 0}+\mathrm{H}_{\mathrm{C}}\right)+\rho_{\mathrm{S}} \mathrm{H}_{\mathrm{S}} / 2\right]
$$

where $\mathrm{P}_{\mathrm{A}}$ is barometric pressure, $\rho_{\mathrm{L}}$ and $\rho_{\mathrm{S}}$ are the liquid and sediment density, respectively, $\mathrm{H}_{\mathrm{L} 0}$ is initial supernatant depth, $\mathrm{H}_{\mathrm{C}}$ is the thickness of the crust if one exists, and $\mathrm{H}_{\mathrm{S}}$ is the sediment depth. Assuming only supernatant is removed, the liquid depth changes by $\Delta \mathrm{H}_{\mathrm{L}}$ and the corresponding pressure change is $\Delta \mathrm{P}=\rho_{\mathrm{L}} \mathrm{g} \Delta \mathrm{H}_{\mathrm{L}}$. Substituting this for $\Delta \mathrm{P}$ in Eq. (3.1.3) and using Eq. (3.1.4) for $\mathrm{P}_{0}$ gives an expression for the supernatant reduction necessary to raise the initial gas volume fraction to neutral buoyancy:

$$
\Delta H_{L}=-\left(\frac{P_{A}}{\rho_{L} g}+H_{L 0}+H_{C}+\frac{\rho_{S}}{\rho_{L}} \frac{H_{S}}{2}\right) \frac{\alpha_{N B}-\alpha_{0}}{\left(1-\alpha_{0}\right) \alpha_{N B}}
$$

If the proposed waste removal is greater than or equal to this depth, a BDGRE can be expected during the operation. According to Eq. (3.1.5), the entire supernatant layer can be removed without inducing a BDGRE in the current population of Waste Group B DSTs except in AN-107. In AN-107 $1.4 \mathrm{~m}(148,000 \mathrm{gal})$ or $19 \%$ of the total supernatant can be removed. However, the increased headspace would move the tank into Waste Group C prior to the BDGRE. 


\subsubsection{Expected Gas Releases During Waste Removal}

Extensive modeling studies based on detailed simulations of the supernatant decant process show with a high degree of confidence that tanks currently in Waste Group A will not reach LFL during waste removal. The simulation results described by Wells et al. (2002) for Waste Group A Tanks AN-103, AN-104, and AN-105 and Waste Group C Tank AW-101 are summarized in Table 3.2. ${ }^{\text {(a) }}$ These results bound the consequences of waste removal from Group B and C tanks.

Table 3.2. Summary of Supernatant Decant Analysis Results

\begin{tabular}{|c|c|c|c|c|}
\hline "Tank & Run & Quantity & Median & $95 \% \mathrm{CL}$ \\
\hline \multirow[t]{8}{*}{$\mathrm{AN}-105$} & Base Case (200 gpm) & Peak Hydrogen (ppm) & 2,800 & 8,300 \\
\hline & & Fraction Gas Release & 0.09 & 0.22 \\
\hline & 30 gpm Decant & Peak Hydrogen (ppm) & 2,600 & 8,000 \\
\hline & & Fraction Gas Release & 0.09 & 0.22 \\
\hline & Stop-Start Control & Peak Hydrogen (ppm) & 2,800 & 8,200 \\
\hline & & Fraction Gas Release & 0.09 & 0.22 \\
\hline & Water Backfill & Peak Hydrogen (ppm) & 23 & 7,600 \\
\hline & & Fraction Gas Release & 0.00 & 0.13 \\
\hline \multirow[t]{8}{*}{ AN-104 } & Base Case & Peak Hydrogen (ppm) & 2,800 & 7,100 \\
\hline & & Fraction Gas Release & 0.12 & 0.27 \\
\hline & 30 gpm Decant & Peak Hydrogen (ppm) & 2,500 & 6,500 \\
\hline & & Fraction Gas Release & 0.12 & 0.27 \\
\hline & Stop-Start Control & Peak Hydrogen (ppm) & 2,800 & 7,000 \\
\hline & & Fraction Gas Release & 0.12 & 0.27 \\
\hline & Water Backfill & Peak Hydrogen (ppm) & 34 & 6,400 \\
\hline & & Fraction Gas Release & 0.00 & 0.16 \\
\hline \multirow[t]{8}{*}{$\mathrm{AN}-103$} & Base Case & Peak Hydrogen (ppm) & 10,500 & 21,100 \\
\hline & & Fraction Gas Release & 0.18 & 0.33 \\
\hline & 30 gpm Decant & Peak Hydrogen (ppm) & 8,200 & 19,100 \\
\hline & & Fraction Gas Release & 0.18 & 0.33 \\
\hline & Stop-Start Control & Peak Hydrogen (ppm) & 9,000 & 19,400 \\
\hline & & Fraction Gas Release & 0.18 & 0.33 \\
\hline & Water Backfill & Peak Hydrogen (ppm) & 1,500 & 21,600 \\
\hline & & Fraction Gas Release & 0.01 & 0.25 \\
\hline \multirow[t]{7}{*}{ AW-101 } & Base Case & Peak Hydrogen (ppm) & 400 & 1,500 \\
\hline & & Fraction Gas Release & 0.05 & 0.17 \\
\hline & 30 gpm Decant & Peak Hydrogen (ppm) & 400 & 1,500 \\
\hline & & Fraction Gas Release & 0.06 & 0.19 \\
\hline & Stop-Start Control & Not run & -- & -- \\
\hline & Water Backfill & Peak Hydrogen (ppm) & 27 & 1,100 \\
\hline & & Fraction Gas Release & 0.00 & 0.09 \\
\hline
\end{tabular}

(a) Tank SY-103 also exhibits BDGREs but is in Waste Group C. It is not scheduled for early retrieval and was not included in the analysis. 
While it is possible for Group B DSTs to experience BDGREs during decant if the change in liquid depth is at least that given by Eq. (3.1.5), the result is expected to be inconsequential. Because the sediment in a Group B tank is, by definition, not near buoyancy, an induced BDGRE can occur only near the end of the transfer if at all. In fact, if the buoyancy ratio is 0.5 or less, no BDGREs can be induced by waste removal. The shallow supernatant depth near the end of transfer reduces the gas release volume, and the larger headspace provides maximum dilution. These factors combine to essentially eliminate the possibility that these late gas releases from Group B tanks would achieve flammability.

Four cases were run for each of the four tanks studied. The base case applied a constant 200-gpm decant rate until all of the supernatant was removed. The headspace ventilation rate was $125 \mathrm{scfm}$ in AW-101 and $100 \mathrm{scfm}$ in the other tanks. The sensitivity to decant rate was tested with a second run using a 30-gpm decant rate. The effect of the normal control strategy was tested with a third run by stopping the decant when the headspace hydrogen concentration exceeded the action level of $6,250 \mathrm{ppm}$ and restarting only when the hydrogen concentration fell below $500 \mathrm{ppm}$. Finally, the efficacy of a proposed mitigation strategy of backfilling with water at the same flow rate as the decant was assessed with a fourth run. It was thought that maintaining the hydrostatic pressure with the backfill might prevent BDGREs. Potential gas release from the floating crust layer via dissolution was not addressed, given its relatively insignificant volume and release rate compared with BDGREs.

Probability distributions of the results were created using a Monte Carlo simulation. The model was run 5,000 or 10,000 times ${ }^{(a)}$ for each case with input parameters for each run selected from probability distributions developed from data and theory. Each simulation represents a complete decant operation. The median and the value at the $95 \%$ confidence level $\left(95^{\text {th }}\right.$ percentile of the cumulative frequency distribution) of the peak hydrogen concentration and the fraction of gas released during decant are given in Table 3.2

Table 3.2 shows that except for AN-103 the peak hydrogen concentrations from BDGREs induced by depressurization approximate those resulting from historic spontaneous events. Base case results for AN-104 and AN-105 were similar, showing a median peak hydrogen concentration of $2,800 \mathrm{ppm}$ with the $95 \%$ confidence level below $25 \%$ of the LFL. The effect of the additional gas retained in AN-104 is counteracted by its larger headspace and lower retained hydrogen fraction. AN-103 contains about twice the gas volume of AN-105, and more of the waste is closer to neutral buoyancy. The result is the highest hydrogen concentrations of the four tanks investigated with a median of $10,500 \mathrm{ppm}$, just exceeding $25 \%$ of the LFL with the $95 \%$ confidence level at 21,100 ppm, about $50 \%$ of the LFL. The gas releases induced in AW-101 were inconsequential due to the shallow sediment layer and low retained hydrogen fraction. The median and 95\% confidence level peak hydrogen concentrations were 400 and 1,500 ppm, respectively.

The gas releases induced in all four tanks were insensitive to decant rates from 30 to 200 gpm. Only AN-103 showed a measurable decrease in peak hydrogen concentration at the median from 10,500 ppm at $200 \mathrm{gpm}$ to $8,200 \mathrm{ppm}$ at $30 \mathrm{gpm}$. Similarly, the stop-start control

(a) All cases for AN-105 and base case runs for the other three tanks used 10,000 simulation runs. The sensitivity cases for tanks other than $\mathrm{AN}-105$ used 5,000 simulation runs. 
strategy did not reduce the peak hydrogen concentrations measurably in any of the four tanks. The initial rise in hydrogen concentration above the action level always resulted from a BDGRE, and the gas release rate and duration of a BDGRE are not affected by decanting once initiated. Therefore, the peak hydrogen concentration was not affected. The main result of the stop-start control strategy was to increase the total time required for decant by a factor of two or three.

Although backfilling with water did not reduce the peak hydrogen concentration at the $95 \%$ confidence level, it caused an important reduction in the mean value. Water backfill cannot prevent a small reduction in hydrostatic pressure, so a few BDGREs were predicted in some of the runs. In runs where BDGREs occurred, the small headspace kept the peak hydrogen concentration higher than it would have been without the backfill. However, except for AN-103, the smaller reduction in hydrostatic pressure completely prevented BDGREs in over half of the runs. This allowed the median peak hydrogen concentration to remain at the background level of 23 to $37 \mathrm{ppm}$. In AN-103, BDGREs were prevented in only about $20 \%$ of the runs, so the median peak hydrogen concentration was lowered to $1,500 \mathrm{ppm}$ from $10,500 \mathrm{ppm}$ but did not fall all the way to background. These results show that the water backfill strategy is probably beneficial. At worst, it does not increase the peak hydrogen concentration over that of the base case. At best, it creates a high probability of having no induced gas releases at all.

\subsubsection{Gas Monitoring Considerations During Waste Removal}

To track the important trends in the headspace hydrogen concentration due to induced BDGREs, gas monitoring must be essentially continuous. Peak hydrogen concentrations typically occur within about an hour of initiation of a BDGRE, and the concentration can exceed the action level in a much shorter time. As noted in Section 3.1.2, BDGREs can potentially be induced during waste removal in DSTs of any waste group. This is not a concern in Group C tanks or when supernatant removal is complete or nearly so, which is the only time BDGREs might occur in Waste Group B tanks. The analysis shows that the current Group A tanks remain below the LFL throughout the transfer but are expected to exceed the action level of 6,250 ppm at the $95 \%$ confidence limit.

It is also possible for delayed BDGREs to occur in Group A tanks after the transfer is shut down. Again, this is not a concern after transfer is complete or nearly so. Because Group A tanks exhibited spontaneous BDGREs at random times prior to waste removal, they should continue to do so during periods when transfer is temporarily halted (e.g., for repairs). In fact, the depressurization that occurred prior to the halt would make a BDGRE even more likely. However, the resulting hydrogen concentrations would be lower than historical norms because removing waste increases the tank headspace and lowers the fraction of gas released per event (see Eq. 3.5.3 and 3.5.4). Delayed releases would generally be smaller than historical norms because of the increased headspace and reduced gas pressure from the waste already removed.

Active ventilation on the order of $100 \mathrm{scfm}$ dilutes the headspace back to near background concentrations within about one day. Because BDGREs occur over a few hours (rapid compared with the ventilation rate), the peak hydrogen concentration during a supernatant decant is not very sensitive to the ventilation rate. The ventilation rate was varied from 10 to $200 \mathrm{scfm}$ in two of the Monte Carlo simulation runs (representing the same complete decant operation but with 
altered input parameters) for the base case in AN-105 that produced a peak hydrogen concentration of $10,000 \mathrm{ppm}$ at $100 \mathrm{scfm}$. The peak hydrogen concentration results are plotted in Figure 3.1. The curve fit through the results indicates that even a very pessimistic passive ventilation rate would not cause the hydrogen concentration to exceed the LFL. On the other hand, increasing the ventilation rate to $500 \mathrm{scfm}$ would only reduce the peak concentration to about $8,000 \mathrm{ppm}$, a $20 \%$ reduction from the base case.

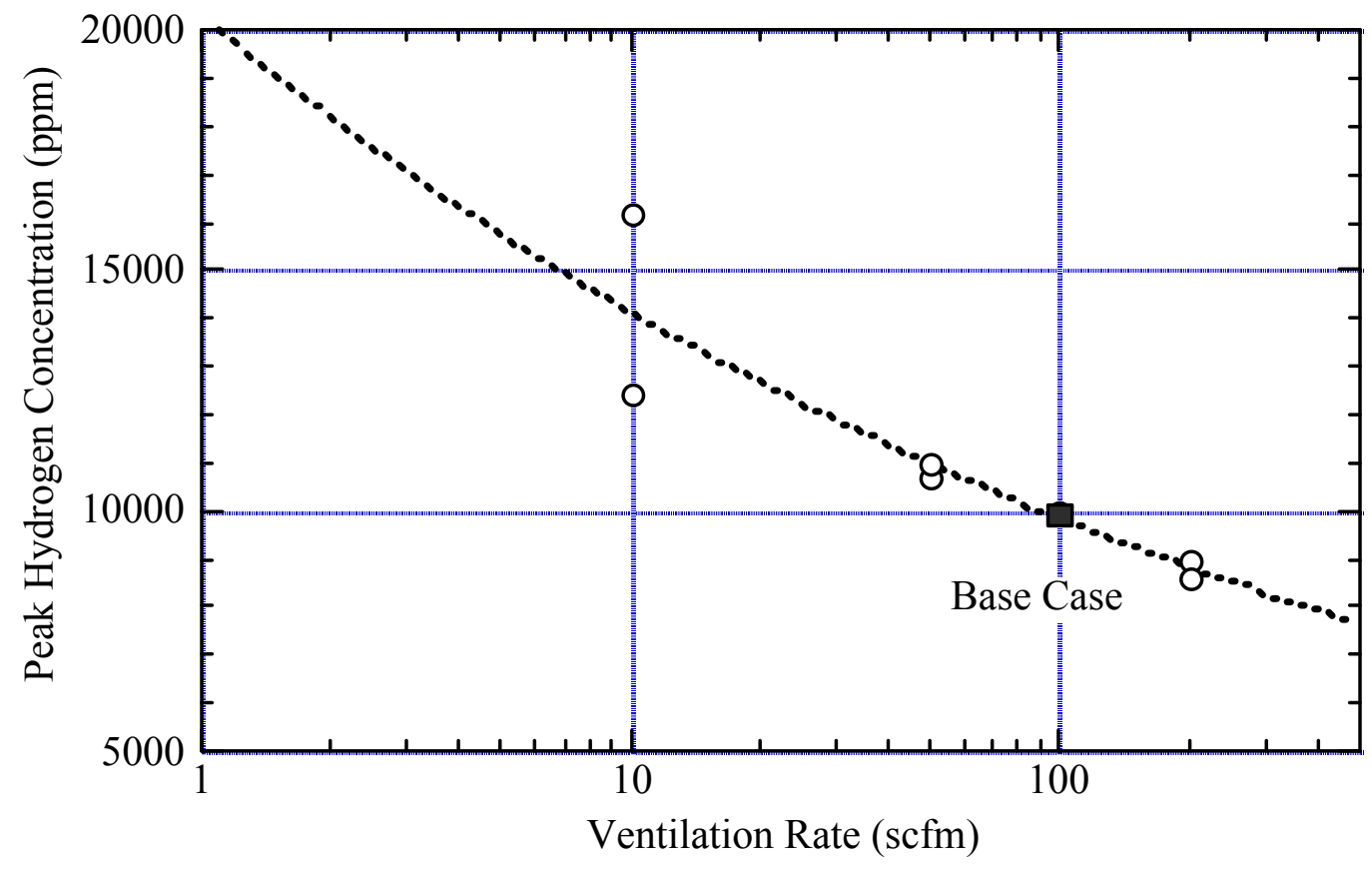

Figure 3.1. Sensitivity of Peak Hydrogen Concentration to Ventilation Rate in AN-105

\subsubsection{Potential Changes in Waste Group Classification After Waste Removal}

Any changes in classification of a tank resulting from waste removal are to a lower waste group. Complete supernatant removal prevents future BDGREs, which would move a tank from Group A to Group B at a minimum. The greatly increased headspace would almost certainly prevent the possibility of reaching flammability if all the retained gas were released, which would move the tank into Group C. It may also be possible to move a tank to Group C for partial supernatant removal if the resulting increase in headspace were sufficient. Because it is not possible for waste removal to move a tank to a higher classification, reanalysis of the waste group classification is optional.

\subsubsection{Potential Changes in Hydrogen Generation After Waste Removal}

The total hydrogen generation rate in the tank is reduced in direct proportion to the fraction of hydrogen generating material removed. In addition, the higher surface-to-volume ratio of the remaining waste will cool and reduce the thermal portion of the total hydrogen generation rate. 
Thus the future steady-state flammability hazard is bounded by the analysis performed on the initial waste condition.

\subsection{Waste Addition}

Fluid waste (liquid waste or liquid-solid slurry) is pumped into a tank so that its actual introduction causes only a local disturbance. Fluid waste may be dumped directly onto the existing waste from a riser or injected under the waste surface through a drop-leg. If a future transfer system were designed to produce high-velocity jets near the tank bottom, mixing would be a concurrent global waste disturbance that should be treated as discussed in Section 3.6. The incoming waste is considered sufficiently concentrated that solids dissolution can be ignored. Saltcake dissolution in SSTs and water addition to both SSTs and DSTs, which also causes dissolution, are covered in Sections 3.4 and 3.5, respectively. Waste addition is specific to DSTs because waste transfers into SSTs are prohibited.

Transfer of waste into a tank causes two kinds of global disturbances. It increases the hydrostatic pressure on the entire waste volume and potentially changes the density of the liquid layer. It also increases the depth of the supernatant and, if slurry is added, the sediment layer.

\subsubsection{Mechanisms for Gas Releases During Waste Addition}

Increasing hydrostatic pressure prevents gas releases by compressing retained gas bubbles and reducing the gas volume fraction. Also, if the density of the incoming fluid is less than the density of the existing supernatant, the neutral buoyancy gas fraction is increased (see Eq. 2.12), which reduces the potential for a BDGRE. However, if the transfer involves a concentrated liquid or a liquid-solid slurry that increases the density of the existing supernatant, the neutral buoyancy gas fraction decreases and a BDGRE may be induced during the addition. As in supernatant decant, the first buoyant displacement can also suspend solids, further reducing the neutral buoyancy gas fraction and possibly inducing additional gas releases.

Other than induced BDGREs, the only mechanisms for gas release during waste addition are ammonia evaporation and release of "hitch-hiker bubbles" from incoming slurry. The rate of ammonia evaporation from a free liquid surface is greatly accelerated by any stirring action induced by waste addition. This effect is intensified if the incoming waste has a high concentration of dissolved ammonia; however, the result is more toxicological than a flammability hazard.

Transfer of a liquid-solid slurry from a gas-retaining tank by mixing, sluicing, or similar method is likely to create small bubbles that attach to particles or are so small they move with the slurry and do not separate until reaching the receiver tank. These "hitchhiker bubbles" create a chronic additional gas release in the receiver tank that is proportional to the transfer rate. However, the total volume of gas and the release rate are not enough to be of concern, as is shown in the next section.

Induced BDGREs are the primary mechanism for consequential gas releases during waste additions. If the incoming fluid is denser than the initial supernatant, the reduction in neutral buoyancy gas fraction quickly overcomes the opposing compression effect and could induce a 
BDGRE. The general principle is shown for a hypothetical waste addition to AN-107 in Figure 3.2, where the average gas volume fraction in the sediment and the neutral buoyancy gas fraction are plotted versus the volume of waste added. The added waste is assumed to have a density of $1,700 \mathrm{~kg} / \mathrm{m}^{3}$ and the initial supernatant a density of $1,370 \mathrm{~kg} / \mathrm{m}^{3}$. Waste addition compresses the gas in the sediment and lowers the gas volume fraction. However, the increasing supernatant density decreases the neutral buoyancy gas fraction much more rapidly. The two curves converge after an addition of about 50,000 gallons - when a BDGRE would occur.

Waste Group A tanks should be most susceptible to BDGREs induced by this mechanism. ${ }^{(a)}$ However, because the initial supernatant density is already quite high in these tanks and most are nearly full, a sizeable reduction in neutral buoyancy void fraction due to waste addition is unlikely. Only additions of slurry with a high solids loading (e.g., if the waste were mixed before or during transfer) could increase the supernatant density and lower the neutral buoyancy void fraction measurably in a receiving Group A tank.

Adding high-density waste to a borderline Group B tank might induce a BDGRE in extreme cases. However, this possibility can be determined beforehand and the added waste volume and density adjusted to prevent it. Because the neutral buoyancy gas fraction is very sensitive to supernatant density, calculating the buoyancy ratio (Eq. 2.13) to find out whether the waste

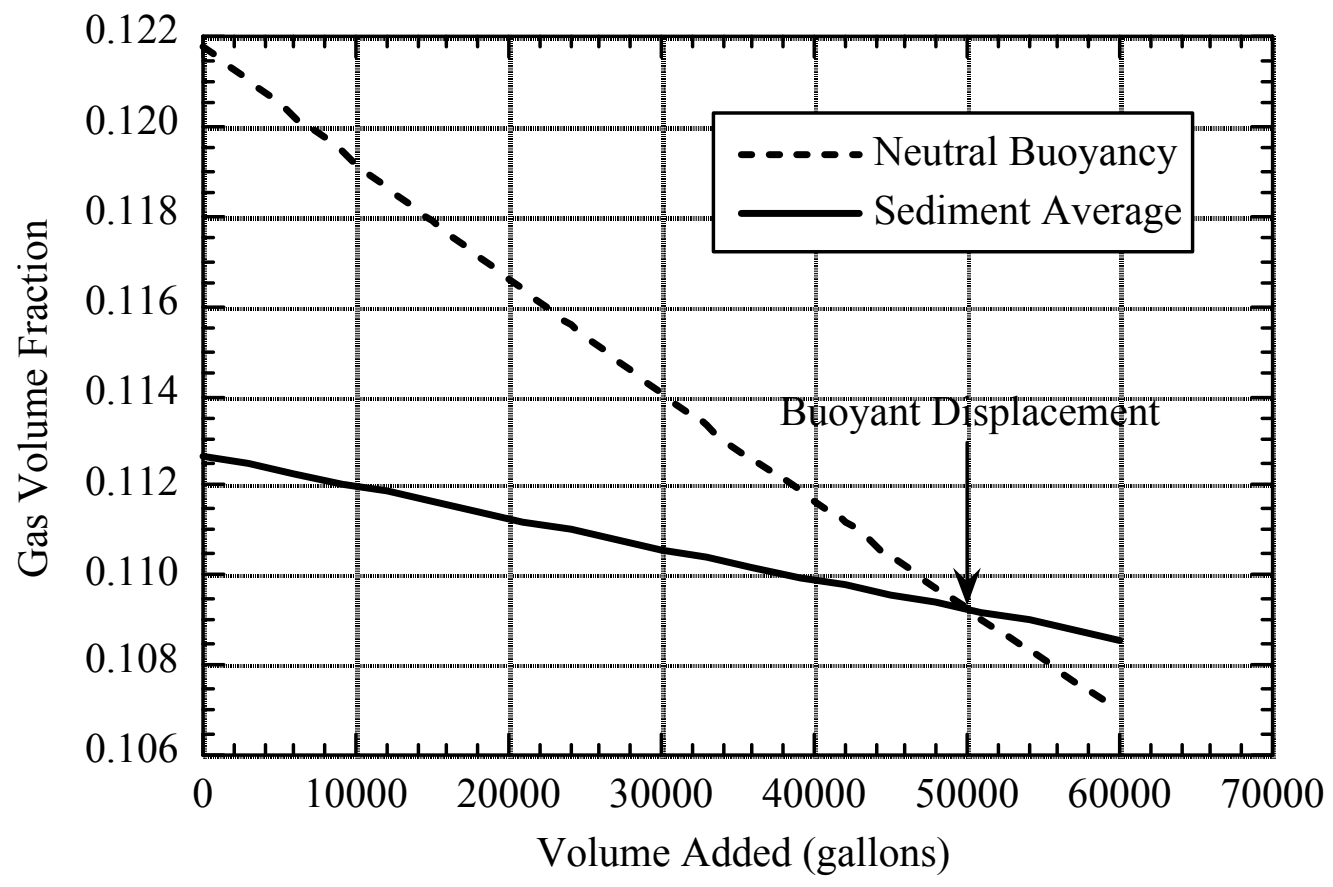

Figure 3.2. Mechanism for Inducing BDGREs During Waste Addition (AN-107)

(a) As described in Section 2.4.1, Waste Group A and B tanks store enough gas to make the headspace flammable if all of it were suddenly released; Waste Group C tanks do not. Waste Group A tanks exhibit spontaneous BDGREs, while tanks in Waste Group B do not. 
addition could move the tank into Waste Group A in the subsequent steady state will also determine whether a BDGRE will occur during the operation, as is shown in the following analysis.

Figure 3.3 illustrates the waste configuration during and after a waste addition. We assume a volume $\mathrm{V}_{\mathrm{T}}$ of waste with density $\rho_{\mathrm{T}}$ is added to the supernatant, which has an initial density $\rho_{\mathrm{L} 1}$ and thickness $\mathrm{H}_{\mathrm{L} 1}$. After mixing, the supernatant will have an increased thickness, $\mathrm{H}_{\mathrm{L} 2}$, and a new density, $\rho_{\mathrm{L} 2}$. The thickness of the gas-retaining sediment layer will decrease from $\mathrm{H}_{\mathrm{S} 1}$ to $\mathrm{H}_{\mathrm{S} 2}$ due to the compression of the retained gas from increased hydrostatic pressure. The gas volume fraction will be reduced from $\alpha_{1}$ to $\alpha_{2}$ in the process.
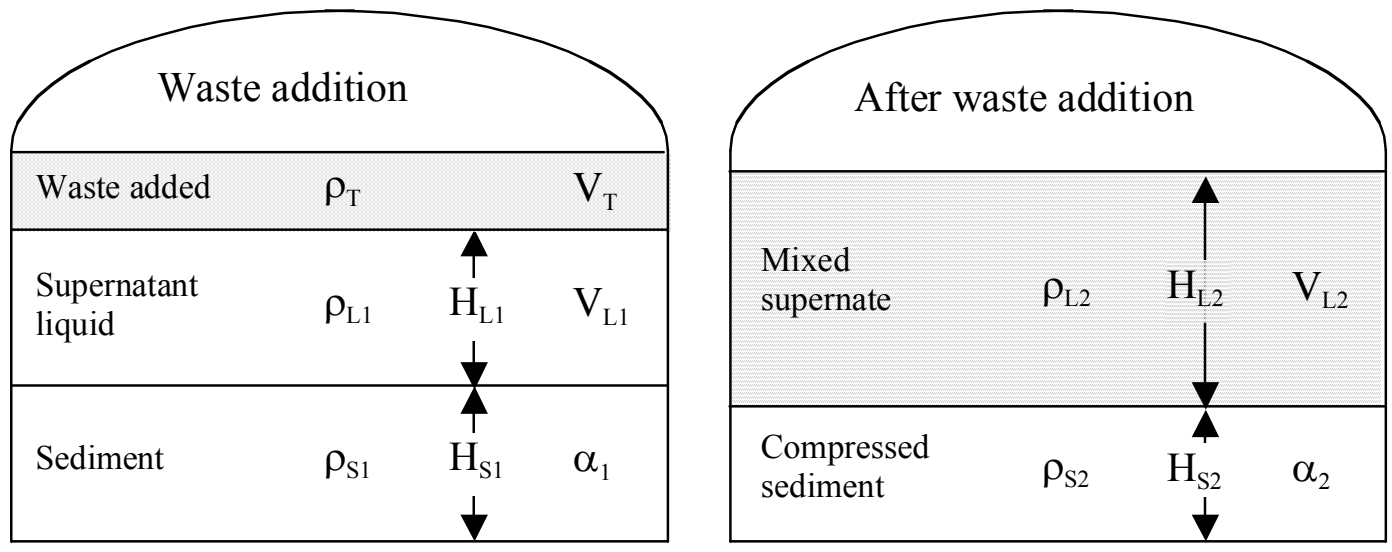

Figure 3.3. Tank Waste Configuration During and After Waste Transfer

Mass conservation for the mixed supernatant liquid after the transfer dictates that

$$
\rho_{\mathrm{L} 2} \mathrm{~V}_{\mathrm{L} 2}=\rho_{\mathrm{L} 1} \mathrm{~V}_{\mathrm{L} 1}+\rho_{\mathrm{T}} \mathrm{V}_{\mathrm{T}}
$$

If we assume constant volume mixing (generally correct to first order, neglecting dissolution or precipitation), then

$$
\mathrm{V}_{\mathrm{L} 2}=\mathrm{V}_{\mathrm{L} 1}+\mathrm{V}_{\mathrm{T}} \text { and } \mathrm{H}_{\mathrm{L} 2}=\mathrm{H}_{\mathrm{L} 1}+\mathrm{V}_{\mathrm{T}} / \mathrm{A}
$$

where $\mathrm{A}$ is the tank cross-sectional area. Combining Eq. (3.2.1) and (3.2.2) results in an expression for the mixture density in terms of the transferred quantities:

$$
\rho_{\mathrm{L} 2}=\frac{\rho_{\mathrm{L} 1} \mathrm{~V}_{\mathrm{L} 1}+\rho_{\mathrm{T}} \mathrm{V}_{\mathrm{T}}}{\mathrm{V}_{\mathrm{L} 1}+\mathrm{V}_{\mathrm{T}}}
$$

The gas volume fraction in the sediment decreases in response to the increase in hydrostatic pressure in accordance with Eq. (3.1.2):

$$
\alpha_{2}=\frac{1}{1+\frac{P_{2}}{P_{1}} \frac{\left(1-\alpha_{1}\right)}{\alpha_{1}}}
$$


The pressure ratio can be determined by applying Eq. (3.1.4) for the average pressure in the sediment. It is useful to ignore the small change in height of the sediment layer so that $\mathrm{H}_{\mathrm{S} 1} \approx \mathrm{H}_{\mathrm{S} 2}$ $=\mathrm{H}_{\mathrm{s}}$. With this simplification, the pressure ratio is found to be

$$
\frac{\mathrm{P}_{2}}{\mathrm{P}_{1}}=\frac{1+\frac{\mathrm{g}}{\mathrm{P}_{\mathrm{A}}}\left[\rho_{\mathrm{L} 2}\left(\mathrm{H}_{\mathrm{L} 2}+\mathrm{H}_{\mathrm{C}}\right)+\rho_{\mathrm{S}} \mathrm{H}_{\mathrm{S}} / 2\right]}{1+\frac{\mathrm{g}}{\mathrm{P}_{\mathrm{A}}}\left[\rho_{\mathrm{L} 1}\left(\mathrm{H}_{\mathrm{L} 1}+\mathrm{H}_{\mathrm{C}}\right)+\rho_{\mathrm{S}} \mathrm{H}_{\mathrm{S}} / 2\right]}
$$

Eq. (3.2.4), along with Eq. (3.2.3) and (3.1.2), gives the final gas volume fraction in terms of the initial conditions and the properties of the added waste.

If the final gas volume fraction is greater than the final neutral buoyancy gas fraction, a BDGRE will occur during the waste addition. That is, if the ratio of the average gas volume fraction to the neutral buoyancy value is greater than unity, a BDGRE will occur. The gas volume fraction at neutral buoyancy is given by Eq. (2.12). Combining this with Eq. (3.1.2) for final gas volume fraction provides an expression for final buoyancy ratio at the end of transfer:

$$
\mathrm{BR}_{2}=\frac{\alpha_{2}}{\alpha_{\mathrm{NB} 2}}=\frac{\alpha_{1} \rho_{\mathrm{S}} /\left(\rho_{\mathrm{S}}-\rho_{\mathrm{L} 2}\right)}{\alpha_{1}+\left(1-\alpha_{1}\right) \mathrm{P}_{2} / \mathrm{P}_{1}}
$$

The buoyancy ratio long after the transfer is complete and the tank reaches its new steady state can also be evaluated directly from Eq. (2.13) to determine whether the result of the waste addition will eventually cause BDGREs and move the tank classification into Waste Group A. Denoting this as $\mathrm{BR}_{3}$,

$$
\mathrm{BR}_{3}=\frac{\alpha_{2}}{\alpha_{\mathrm{NB} 2}}=\frac{\mathrm{C}_{\mathrm{B}}}{\rho_{\mathrm{S}}-\rho_{\mathrm{L} 2}}\left(\frac{\mathrm{GT}}{\mathrm{P}_{2}}\right)^{1 / 3} \mathrm{H}_{\mathrm{S}}^{2}
$$

The final density is calculated with Eq. (3.2.3) and the final pressure with Eq. (3.1.4). The small change in sediment depth is ignored, and the temperature and gas generation rate in the sediment are assumed unaffected by the waste addition. Because the long-term steady-state buoyancy ratio, $\mathrm{BR}_{3}$, via Eq. (3.2.6) is always greater than that determined from the process model of Eq. (3.2.5), a determination that there will be no change in waste group (i.e., $\mathrm{BR}_{3}>1$ ) also ensures that no BDGREs will occur during the process (i.e., $\alpha_{2}<\alpha_{\mathrm{NB} 2}$ ). To demonstrate this, we recast Eq. (3.2.5) for the buoyancy ratio at the end of transfer in terms of the initial buoyancy ratio prior to transfer by substituting for the initial gas volume fraction, $\alpha_{1}$, in the numerator:

$$
\mathrm{BR}_{2}=\frac{1}{\alpha_{1}+\left(1-\alpha_{1}\right) \mathrm{P}_{2} / \mathrm{P}_{1}} \mathrm{BR}_{1} \frac{\rho_{\mathrm{S}}-\rho_{\mathrm{L} 1}}{\rho_{\mathrm{S}}-\rho_{\mathrm{L} 2}}
$$


The analog to Eq. (3.2.7) for the buoyancy ratio long after transfer is computed via Eq. (3.2.6) as

$$
\mathrm{BR}_{3}=\mathrm{BR}_{1} \frac{\rho_{\mathrm{S}}-\rho_{\mathrm{L} 1}}{\rho_{\mathrm{S}}-\rho_{\mathrm{L} 2}}\left(\frac{\mathrm{P}_{1}}{\mathrm{P}_{2}}\right)^{1 / 3}
$$

The ratio of long-term to short-term buoyancy ratios is greater than unity except for a very large initial gas fraction, as shown by

$$
\mathrm{BR}_{3} / \mathrm{BR}_{2}=\left[\alpha_{1}+\left(1-\alpha_{1}\right) \mathrm{P}_{2} / \mathrm{P}_{1}\right]\left(\frac{\mathrm{P}_{1}}{\mathrm{P}_{2}}\right)^{1 / 3}>1 \text { for } \alpha_{1}>2 / 3
$$

Temperature and gas volume are also related. Adding higher-temperature waste would increase the sediment gas volume fraction and could potentially cause it to become buoyant. Assuming a constant pressure and instantaneous heat transfer from the mixed liquid layer into the sediment, the change in gas volume fraction due to a change in temperature is expressed analogously to Eq. (3.1.2) by

$$
\alpha_{2}=\frac{1}{1+\frac{T_{1}}{T_{2}} \frac{\left(1-\alpha_{1}\right)}{\alpha_{1}}}
$$

where $T_{1}$ and $T_{2}$ are the absolute temperatures before and after waste addition, respectively. With an initial gas volume fraction of 0.1 and an initial temperature of $100^{\circ} \mathrm{F}$, an increase in the sediment temperature of $65^{\circ} \mathrm{F}$ would be required to raise the gas fraction $10 \%$. This means that the initial buoyancy ratio would have to be 0.9 or greater to cause a BDGRE. We conclude that temperature changes are not an important issue in gas releases during waste addition.

\subsubsection{Expected Gas Releases During Waste Addition}

No specific analysis has been performed on gas release rates and volumes of BDGREs induced in Group A tanks during waste addition. Because the initiating mechanism is sediment buoyancy, as it is in spontaneous releases, BDGRE gob size would be expected to follow historic norms. However, the peak hydrogen concentration would be higher because waste addition decreases the headspace and increases the gas release fraction by raising the hydrostatic pressure (see Eq. 3.5.3 and 3.5.4).

There has been no experience or analysis on the size of BDGREs induced in Group B or C tanks that have not exhibited them in the past. In the absence of historic BDGREs the waste should be more uniform, so the first induced BDGRE might be larger than indicated by the behavior of the current Group A tanks. However, if the waste addition is adjusted to prohibit BDGREs, no appreciable gas release is expected from any other mechanism. 
Waste addition tends to increase ammonia evaporation by disturbing the supernatant and refreshing the liquid surface. Ammonia concentrations as high as 7,000 ppm were observed in SY-102 while it was receiving waste from SY-101 (Mahoney et al. 2000). Ammonia was also observed to increase to about 1,500 ppm in SY-102 while it received saltwell liquor.

The "hitchhiker" bubble phenomenon apparently caused hydrogen concentrations to rise in AY-102 when it was receiving sludge sluiced from C-106 (Cuta et al. 1999). The hydrogen concentration in SY-102 increased to about $240 \mathrm{ppm}$ when receiving mixed slurry from SY-101 (Mahoney et al. 2000). However, the gas release rates in both these cases were relatively low, and there was insufficient gas in the entire transfer to raise the hydrogen concentration to the action level, even with zero ventilation. Hitchhiker bubbles were determined to be a negligible effect in the brine transferred from tanks being saltwell pumped (Peurrung et al. 1998). No hydrogen elevation was detected in SY-102 while it received saltwell liquor.

\subsubsection{Gas Monitoring Considerations During Waste Addition}

BDGREs induced by any operation occur over a relatively short period that requires essentially continuous monitoring to capture the approach to peak hydrogen concentration. However, in the case of waste addition, the calculations outlined in Section 3.2.1 can be applied to adjust a given transfer to avoid creating a tank exhibiting spontaneous BDGREs, which also prevents BDGREs from occurring during the transfer.

Transfers of waste from tanks in which the waste has been mixed or otherwise agitated can carry hitchhiker gas bubbles that are released in the receiver tank. If the ventilation system failed while the transfer continued, the hydrogen concentration could exceed the action level, though it is doubtful that the LFL would be reached. Because the mechanics of saltwell pumping separates the retained gas bubbles from the brine, and because of the very low transfer rate, hitchhiker bubbles are not an issue in tanks receiving saltwell liquor, even without active ventilation. To quantify these assertions, the simple headspace mass conservation model developed to study gas releases during saltcake dissolution (Stewart 2001) and discussed in Section 3.4.3 is simplified to consider only inflow of waste to a tank. The headspace hydrogen concentration is expressed as a function of time as

$$
\mathrm{C}_{\mathrm{H}}(\mathrm{t})=\frac{\mathrm{Q}_{\mathrm{GAS}} \chi_{\mathrm{H}}}{\mathrm{Q}_{\mathrm{VIN}}+\mathrm{Q}_{\mathrm{GAS}}}+\left(\mathrm{C}_{0}-\frac{\mathrm{Q}_{\mathrm{GAS}} \chi_{\mathrm{H}}}{\mathrm{Q}_{\mathrm{VIN}}+\mathrm{Q}_{\mathrm{GAS}}}\right)\left(1-\frac{\mathrm{Q}_{\mathrm{TIN}}}{\mathrm{V}_{\mathrm{HS} 0}}\right)^{\frac{\mathrm{Q}_{\mathrm{VIN}}+\mathrm{Q}_{\mathrm{GAS}}}{\mathrm{Q}_{\mathrm{TIN}}}}
$$

where

$\mathrm{C}_{0} \quad=$ initial headspace hydrogen concentration

$\mathrm{V}_{\mathrm{HSO}}=$ initial headspace volume

$\chi_{\mathrm{H}}=$ hydrogen fraction of the retained gas in the waste

$\mathrm{Q}_{\mathrm{GAS}}=$ gas release rate from bubbles in the transfer stream

$\mathrm{Q}_{\mathrm{VIN}}=$ estimated headspace passive (or measured active) ventilation rate

$\mathrm{Q}_{\mathrm{TIN}}=$ incoming transfer flow rate.

As a bounding case, consider an empty DST being filled with slurry containing a gas volume fraction of 0.03 . This was the maximum value attributed to the SY-101 mixed slurry transferred 
to SY-102 during remediation and is likely to be the bounding value for any transfer. Assume the gas contains $60 \%$ hydrogen, twice the concentration in SY-101 waste but representative of other DSTs that might be mixed prior to transfer. The peak hydrogen concentration occurs when the tank is filled to the maximum level, assumed to be 422 inches. At a transfer rate of $100 \mathrm{gpm}$ with no in-line dilution, the tank would be filled in eight days, and Eq. (3.2.9) predicts a peak hydrogen concentration of $2,400 \mathrm{ppm}$ at a 100 -scfm ventilation rate. Using a passive ventilation rate of $2.5 \mathrm{scfm}$, conservatively representative of a failed active ventilation system (Hu 2002), the peak hydrogen concentration would be $26,000 \mathrm{ppm}$ and the action level of $6,250 \mathrm{ppm}$ would have been exceeded in 70 hours (about three days). With zero ventilation, the peak hydrogen concentration would be $30,000 \mathrm{ppm}$, still under the LFL for hydrogen alone. A concurrent ammonia concentration of over 50,000 ppm would be necessary to reach $100 \%$ of the LFL.

For the lower transfer rates of saltwell pumping, a much longer time is required, and the hydrogen concentration is much more sensitive to ventilation. Still assuming the bounding gas loading in the transfer stream but a $10 \mathrm{gpm}$ transfer rate (might represent three or four SSTs being saltwell pumped simultaneously), the peak hydrogen concentration is only $240 \mathrm{ppm}$ for a $100 \mathrm{scfm}$ ventilation rate. For 2.5 -scfm passive ventilation, the peak is $9,000 \mathrm{ppm}$ after 81 days, exceeding the action level of $6,250 \mathrm{ppm}$ in 43 days. Zero ventilation gives the same 30,000-ppm peak as the 100-gpm transfer, while the action level is exceeded in 29 days. If completely filling a sealed (zero ventilation) empty tank with a highly gassed slurry reaches only $75 \%$ of the LFL, smaller transfers with lower gas loading pose no hazard with or without ventilation.

\subsubsection{Potential Changes in Waste Classification After Waste Addition}

As described in Section 3.2.1, it is possible to reduce the neutral buoyancy gas fraction enough that a Group B or C tank could eventually experience BDGREs and become a Group A tank. However, a very large waste addition at high density would be required. Figures 3.4 and 3.5 show the results of buoyancy ratio calculations using Eq. (2.13) for various transfer volumes and densities into Tank AW-105, now in Waste Group C, and AN-107, which is in Waste Group B. Table 3.3 shows the parameters used in the calculations for each tank. The added waste volume in the calculations was limited to that required to raise the waste level to about $10 \mathrm{~m}$.

Waste Group C Tank AW-105 has a very low initial supernatant density, so adding highdensity waste has a strong effect. About 70,000 gallons of additional waste at a density of $1,500 \mathrm{~kg} / \mathrm{m}^{3}$ has the potential to make this tank exhibit BDGREs. However, the headspace reduction of this small addition would probably not be sufficient to move the tank out of Waste Group C. If the incoming density is reduced to $1,300 \mathrm{~kg} / \mathrm{m}^{3}$, the buoyancy ratio reaches unity at about 280,000 gallons of waste addition. At this point, the headspace is about $75 \%$ of the initial volume, so it might now become flammable if all of the retained gas were released, and the tank would move to Waste Group A. With a density of $1,200 \mathrm{~kg} / \mathrm{m}^{3}$, the buoyancy ratio does not approach unity with even the maximum possible addition, though the greatly reduced headspace after a very large transfer would likely place the tank in Waste Group B. 


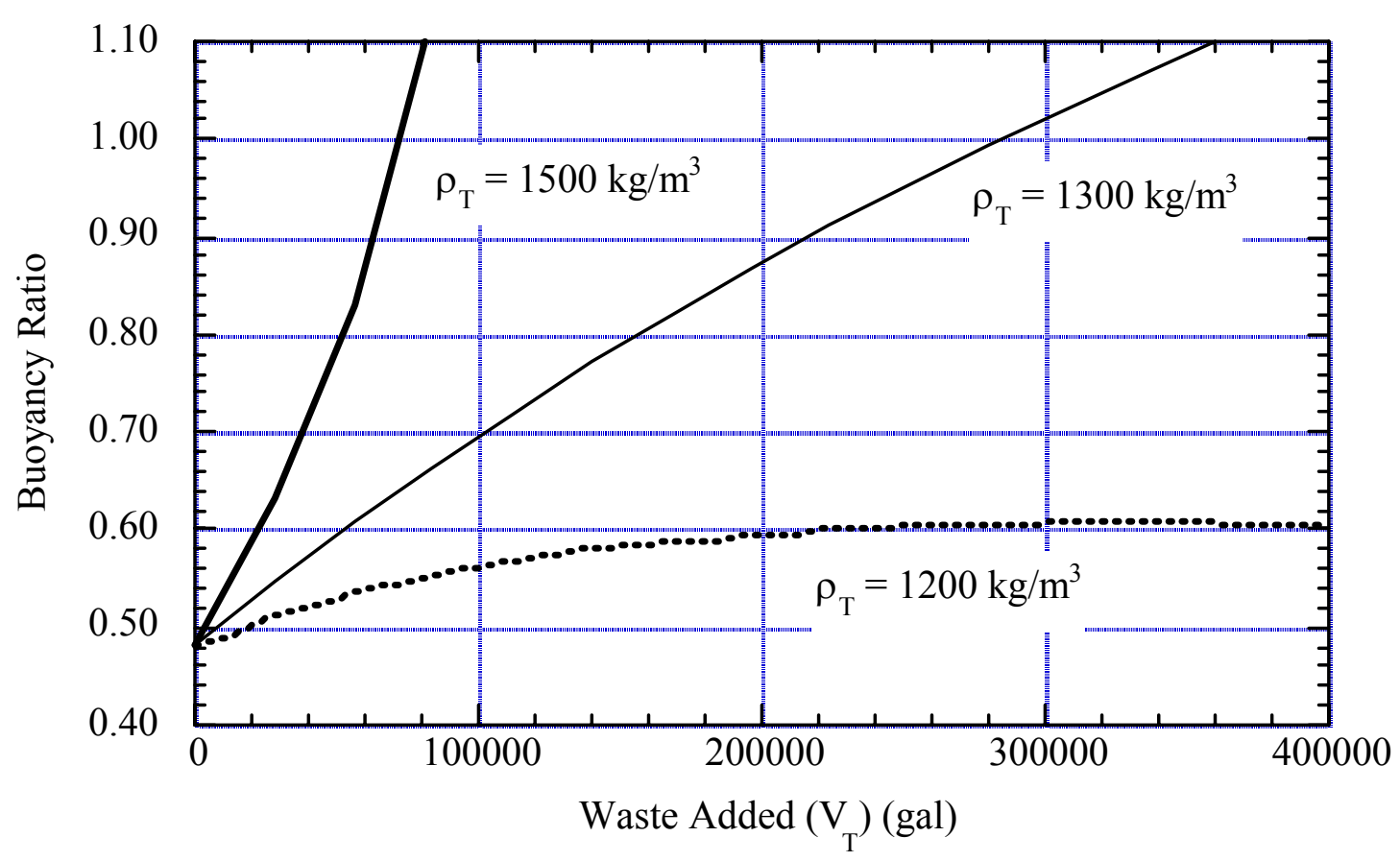

Figure 3.4. Effects of Waste Addition on Buoyancy Ratio for Tank AW-105

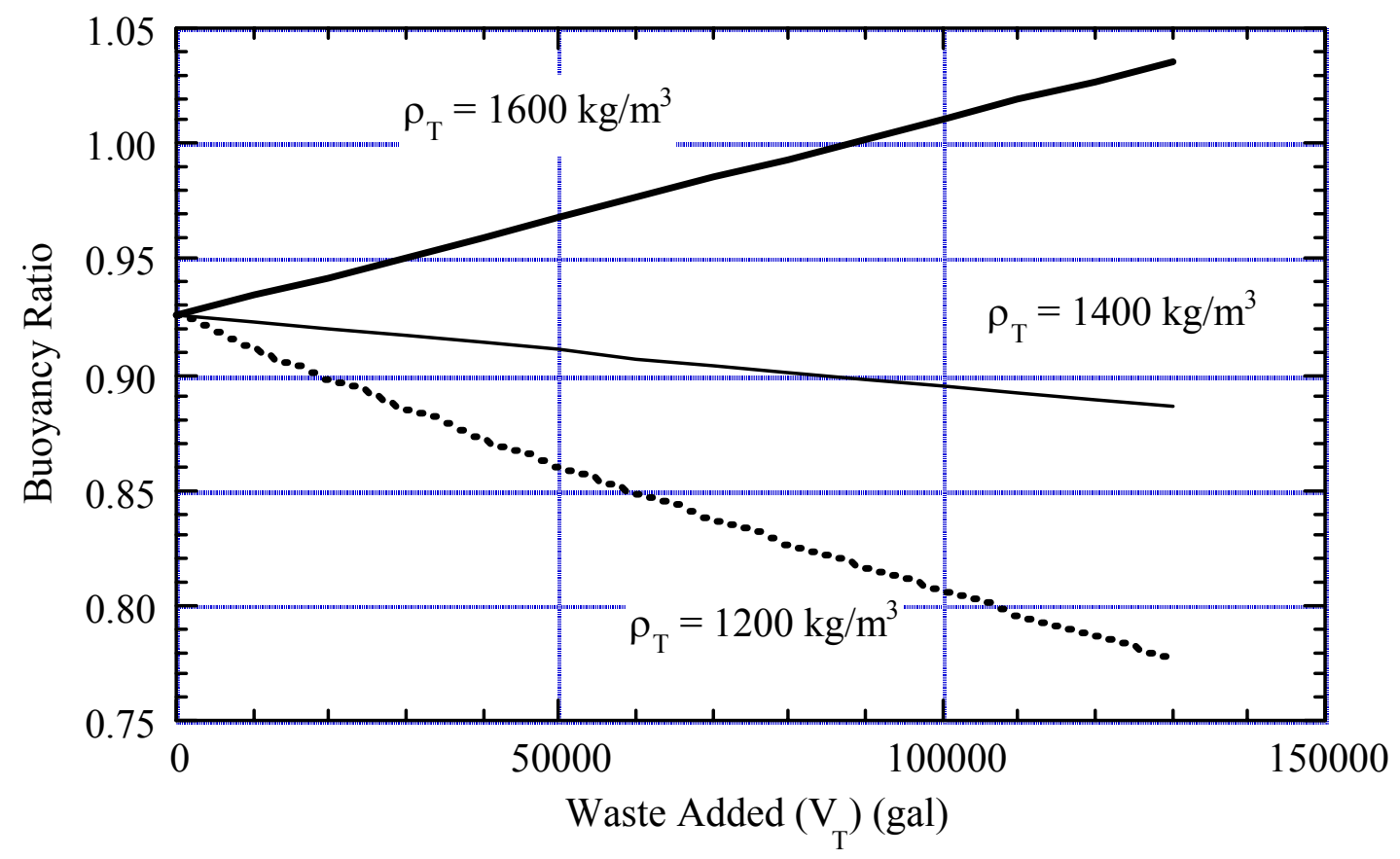

Figure 3.5. Effects of Waste Addition on Buoyancy Ratio for Tank AN-107 
Table 3.3. Parameters Used for Waste Transfer Analysis (Barker and Hedengren 2002)

\begin{tabular}{|c|c|c|}
\hline Property & AW-105 (Group C) & AN-107 (Group B) \\
\hline $\mathrm{H}_{\mathrm{L} 1}(\mathrm{~m})$ & 1.6 & 7.3 \\
\hline $\mathrm{H}_{\mathrm{S}}(\mathrm{m})$ & 2.4 & 2.3 \\
\hline$\rho_{\mathrm{L} 1}\left(\mathrm{~kg} / \mathrm{m}^{3}\right)$ & 1070 & 1370 \\
\hline$\rho_{\mathrm{S}}\left(\mathrm{kg} / \mathrm{m}^{3}\right)$ & 1306 & 1560 \\
\hline$\alpha_{\mathrm{NB} 1}$ & 0.18 & 0.12 \\
\hline $\mathrm{G}\left(\mathrm{mol} / \mathrm{m}^{3} /\right.$ day) & 0.0017 & 0.0116 \\
\hline $\mathrm{T}(\mathrm{K})$ & 293 & 305 \\
\hline $\mathrm{BR}_{1}$ & 0.48 & 0.93 \\
\hline
\end{tabular}

The waste level in AN-107 is higher than in AW-105, so only about 120,000 gallons may be added. The supernatant is much less dilute, so only the densest waste addition, $1,600 \mathrm{~kg} / \mathrm{m}^{3}$, can decrease the neutral buoyancy gas fraction and, because the sediment density is higher than in AW-105, the effect is less pronounced. The buoyancy ratio will exceed unity in this tank if the maximum addition amount of 120,000 gallons of waste with a density of $1,600 \mathrm{~kg} / \mathrm{m}^{3}$ are added.

These examples show that it is possible for a waste addition to elevate a tank's classification from Waste Group C or B to A, where the tank may exhibit BDGREs and have a sufficiently small headspace that a sudden release of the entire gas inventory could make it flammable. This points out the need for tank classification analyses prior to any waste addition to ensure the proper controls are in place prior to moving a Group C tank into Waste Group B and adjusting the operation to prevent moving a tank into Waste Group A.

While additions to Waste Group A tanks cannot raise their classification, the reduced headspace will increase the hydrogen concentration resulting from continuing spontaneous BDGREs. Also, the increased liquid depth and consequent increased hydrostatic pressure will increase the volume of gas released in a BDGRE. Any solids that are transferred in add to the sediment depth, providing a larger total retained gas volume. All these effects combine to exacerbate the consequences of existing BDGREs.

\subsubsection{Potential Changes in Hydrogen Generation After Waste Addition}

By definition, adding waste to a tank increases its total hydrogen generation rate and reduces its headspace. Both will exacerbate steady-state hydrogen concentration and time to flammability. Therefore, the steady-state hydrogen generation rate must be reassessed for any waste addition.

\subsection{Saltwell Pumping}

Saltwell pumping is the primary method for removing drainable liquids from the SSTs to prevent or reduce the consequences of a leak. In this method, a long cylindrical metal screen (the saltwell screen) is installed as a well casing near the center of the tank and extends virtually 
to the bottom of the tank. A jet pump located inside and at the bottom of the saltwell screen pumps liquid out as it drains into the saltwell screen.

Saltwell pumping is a gradual process. At the onset of pumping, supernatant flows freely into the well and the pumping rate is limited by pump capacity. After the supernatant is exhausted, the rate at which brine enters the saltwell screen slows as brine drains through the waste. The pumping rate is then reduced to approximately match the liquid drainage rate. The pumping campaign is complete when the pumping rate falls below some predetermined value (less than $1 / 2$ gpm).

Recent campaigns have been as short as three months (Tank S-103, from which less than 24,000 gallons of liquid were removed) and as long as a year (Tank SX-104, from which about 117,000 gallons of liquid were removed). The duration of saltwell pumping in any given tank is a function of the amount of drainable liquid, the drainage rate (which depends on permeability), and the actual times the system is available for pumping (stoppages for corrective and preventive maintenance are common).

Besides the removal of liquid, the most important global waste disturbance of saltwell pumping in saltcake waste is the increased lithostatic load in the waste column as the buoyant force of the interstitial liquid is removed. This creates a new waste configuration with an unsaturated layer of waste overlying a saturated zone. Only the saturated regions of the sediment are capable of retaining flammable gas and, as described in Section 2, the increased lithostatic load changes the retained gas configuration to a form with a different release behavior. The increased lithostatic load also leads to compaction and subsidence of the central portion of the tank that may eventually create a broad crater or depression. Sludge gives up liquid by consolidation rather than draining, and neither the waste configuration nor the gas retention mechanism changes during pumping. The rest of this section concerns pumping of saltcake tanks.

\subsubsection{Mechanisms for Gas Release During Saltwell Pumping}

Removing supernatant and interstitial liquid by saltwell pumping can induce gas releases by several mechanisms. The hydrostatic pressure within the waste decreases as liquid is removed, which causes trapped gas bubbles to expand and dissolved gases to evolve into the bubbles. Bubble growth from both mechanisms can cause bubble disengagement or percolation gas release (see Section 2.2). Particle-displacing bubbles that were not released by these mechanisms "pop" when the interstitial liquid drains away from around them (Peurrung et al. 1997). However, some liquid remains after the bulk of it has been drained away, and some gas will remain trapped as small, pore-filling bubbles surrounded by this undrainable liquid. Bubbles in this condition are eventually released when the entrapping liquid evaporates or gradually drains away.

The evaporation of moisture that is held up in the unsaturated waste after initial draining is a source of ammonia and other soluble gases (Peurrung et al. 1997). Because this liquid is trapped in small crevices between particles, its surface area is huge and the evaporation rate can be high. 
However, the evolution of these gases has not proven to be a significant flammability hazard but more of a toxicological concern.

\subsubsection{Expected Gas Releases During Saltwell Pumping}

Gas is released from the waste as it becomes unsaturated so the gas release rate roughly follows the pumping rate. Hedengren et al. (2001) give a summary of observed gas release behavior during saltwell pumping based on headspace gas monitoring data. The correlation between saltwell pumping and gas release is illustrated in Figure 3.6, where the hydrogen release rate in Tank U-105 is plotted along with the daily volume of liquid pumped. Headspace ventilation rates were determined from a coincident tracer gas study and used with headspace hydrogen and nitrous oxide concentrations to estimate their release rates. ${ }^{\text {(a) }}$ In Figure 3.6, we see about a one-day lag between pumping activity and gas release. But the lag is greater in several cases, and pumping activities are not always accompanied by increased gas release rates. This suggests that the timing and magnitude of gas releases are also subject to factors other than pumping.

Similar correlations between pumping and gas release are also observed in headspace gas monitoring data in other tanks (Huckaby et al. 1999). ${ }^{\text {(a) }}$ The highest gas release rates occur shortly after the onset of pumping, when the waste drains rapidly and pumping rates tend to be high. This is illustrated in Figure 3.7, where the estimated hydrogen release rate ${ }^{(b)}$ has been

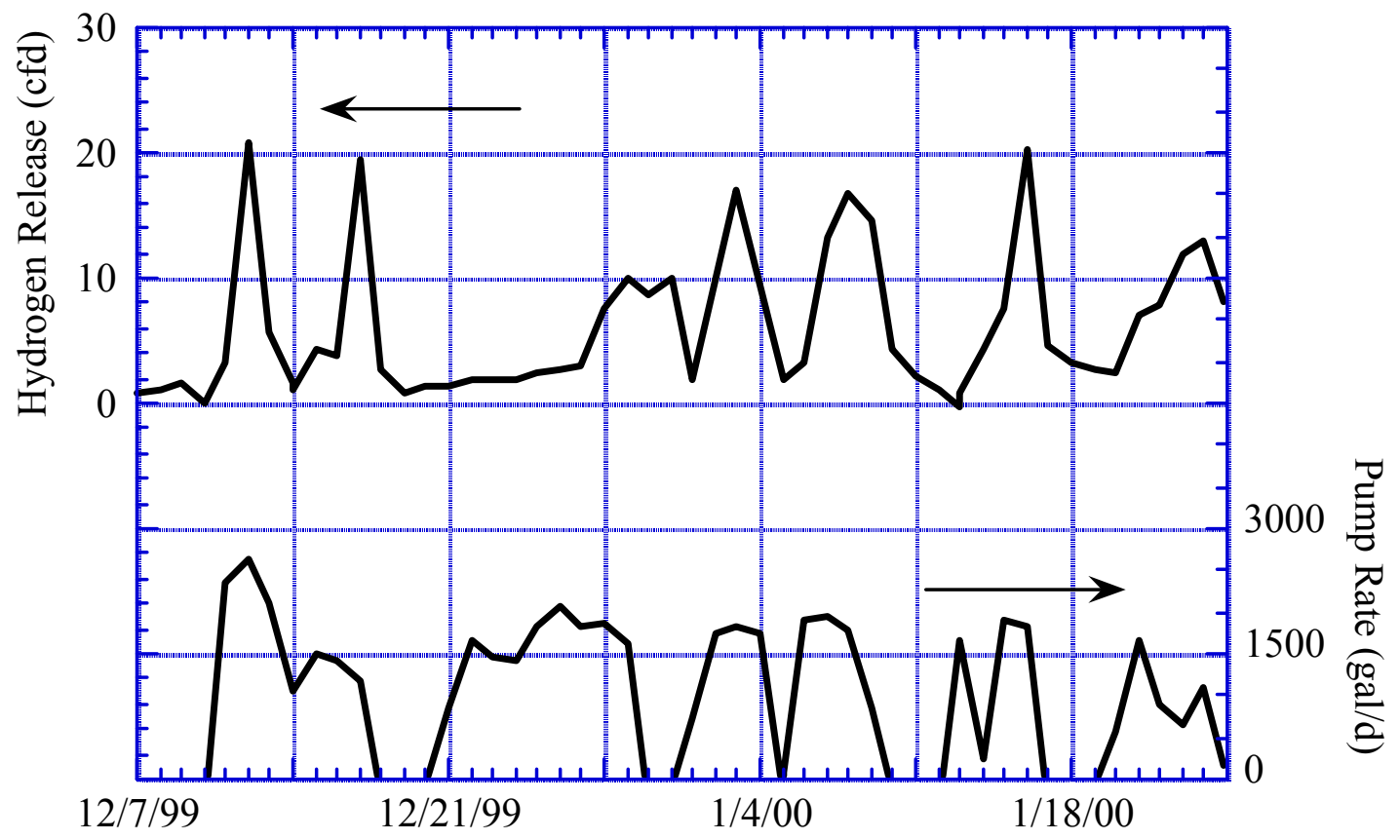

Figure 3.6. U-105 Hydrogen Release and Saltwell Pumping Rates

(a) Peurrung LM and JL Huckaby. March 10, 2000. Gas Release Behavior During Saltwell Pumping. Letter Report TWS00.39, Pacific Northwest National Laboratory, Richland, WA.

(b) The negative gas release rates in Figure 3.7 are not physically reasonable and indicate that the actual ventilation rate at those times was considerably higher than the $9.1 \mathrm{ft}^{3} /$ min used in calculations. 


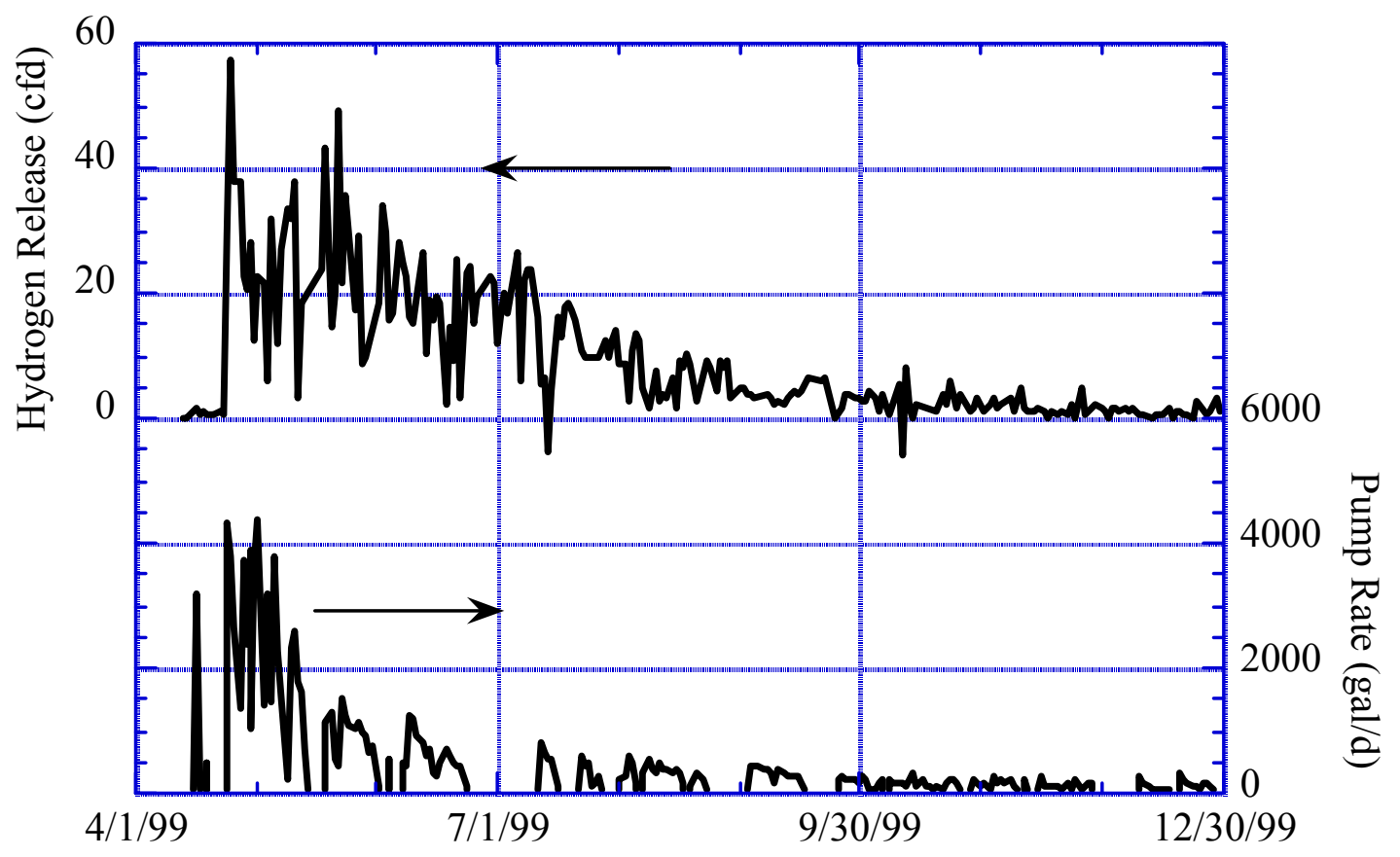

Figure 3.7. Tank 241-S-106 Estimated Hydrogen Release Rate and Pumping Rate

plotted above the pumping rate for S-106. Much of the liquid being pumped in this initial period consists of supernatant and interstitial brine immediately surrounding the saltwell screen that drains quickly. During this phase, starting and stopping the saltwell pump can cause gas release rates to rise and fall rapidly, as shown in Figure 3.6 for U-105.

Continued pumping exhausts the supernatant and depletes the region near the saltwell screen of liquid; thus the drainage rate decreases. Gas release rates decline correspondingly, as indicated in Figure 3.7. In this second phase of pumping, the liquid saturation interface (or interstitial liquid level) rises gradually from a low point at the saltwell to a higher level near the tank wall. The majority of liquid being pumped during this phase comes from the regions away from the saltwell screen. A temporary cessation in pumping has little immediate effect on drainage rate far from the saltwell, and the gas release rate is thus more independent of pumping rate.

After pumping ceases, drainage continues from the outer regions of the waste toward the saltwell screen as well as from the upper regions of the waste that had not drained completely. This continues to release trapped gases, albeit slowly. The headspace hydrogen and nitrous oxide concentrations in Tank S-106, for example, appear to have remained high for months after saltwell pumping was stopped. ${ }^{(a)}$

Because ammonia is highly soluble in liquid wastes, headspace ammonia concentrations are not expected to rise and fall as pumping starts and stops (Peurrung et al. 1997). A reservoir of

(a) Peurrung LM and JL Huckaby. March 10, 2000. Gas Release Behavior During Saltwell Pumping. Letter Report TWS00.39, Pacific Northwest National Laboratory, Richland, WA. 
dissolved ammonia exists where the waste itself remains wet; it releases ammonia vapor to the headspace as mass transport allows. Saltwell pumping apparently did not affect the ammonia concentration in Tank U-105 until the supernatant was removed; then the increased wetted surface area (associated with the exposed porous waste surface) caused a rapid rise in ammonia concentration (Figure 3.8). ${ }^{(a)}$

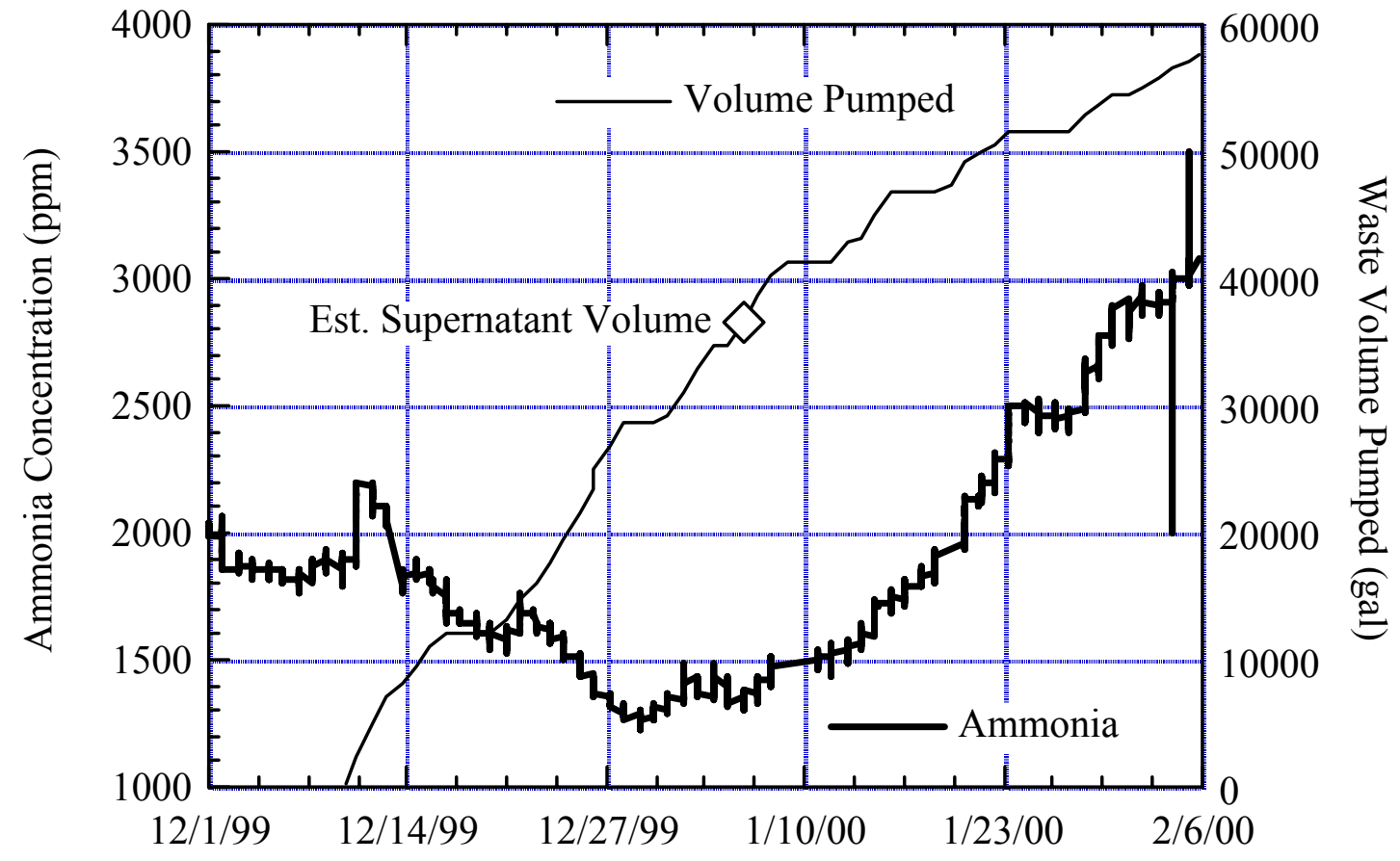

Figure 3.8. U-105 Ammonia Release and Waste Volume Pumped

The overall result of the saltwell pumping process in saltcake tanks is the release of a substantial fraction of the retained gas, though it is impossible to quantify it accurately. Approximate integration of the hydrogen release rate over the pumping period in S-106 indicates a total gas release of $160 \pm 40 \mathrm{~m}^{3}$ (Hedengren et al. 2001). This volume ranges from 30 to $70 \%$ of the total retained gas volume estimated by several methods. Approximately half of the retained gas volume in U-105 was released by saltwell pumping. ${ }^{(a)}$ Similar fractions could be expected in other saltcake tanks. As discussed in Section 2.3.4, sludge tanks are not expected to release a significant fraction of their retained gas during saltwell pumping.

\subsubsection{Gas Monitoring Considerations During Saltwell Pumping}

Hydrogen concentration measurements in SSTs from 1995 to 2001 (McCain 2001) show that gas releases induced by saltwell pumping are unimportant in terms of flammability. The highest hydrogen gas concentration ever measured by standard hydrogen monitoring systems (SHMS) in an SST was 7,200 ppm, detected in BY-106 during saltwell pumping in 1995 (Watrous et al.

(a) Huckaby JL, LA Mahoney, and ER Siciliano. 2001. Waste Gas Releases Associated with Recent Saltwell Pumping of Single-Shell Tanks. TWS01.036, Pacific Northwest National Laboratory, Richland, WA. 
2000). U-103, U-105, and U-109 had hydrogen concentrations approaching 5,000 ppm during pumping in 1999 and early 2000. Saltwell pumping in S-111 was shut down on February 7, 2002 when the hydrogen concentration passed 5,500 ppm; it eventually reached a peak of 6,600 ppm on February 11. The headspace hydrogen concentration and waste level for the period of interest are plotted in Figure 3.9.

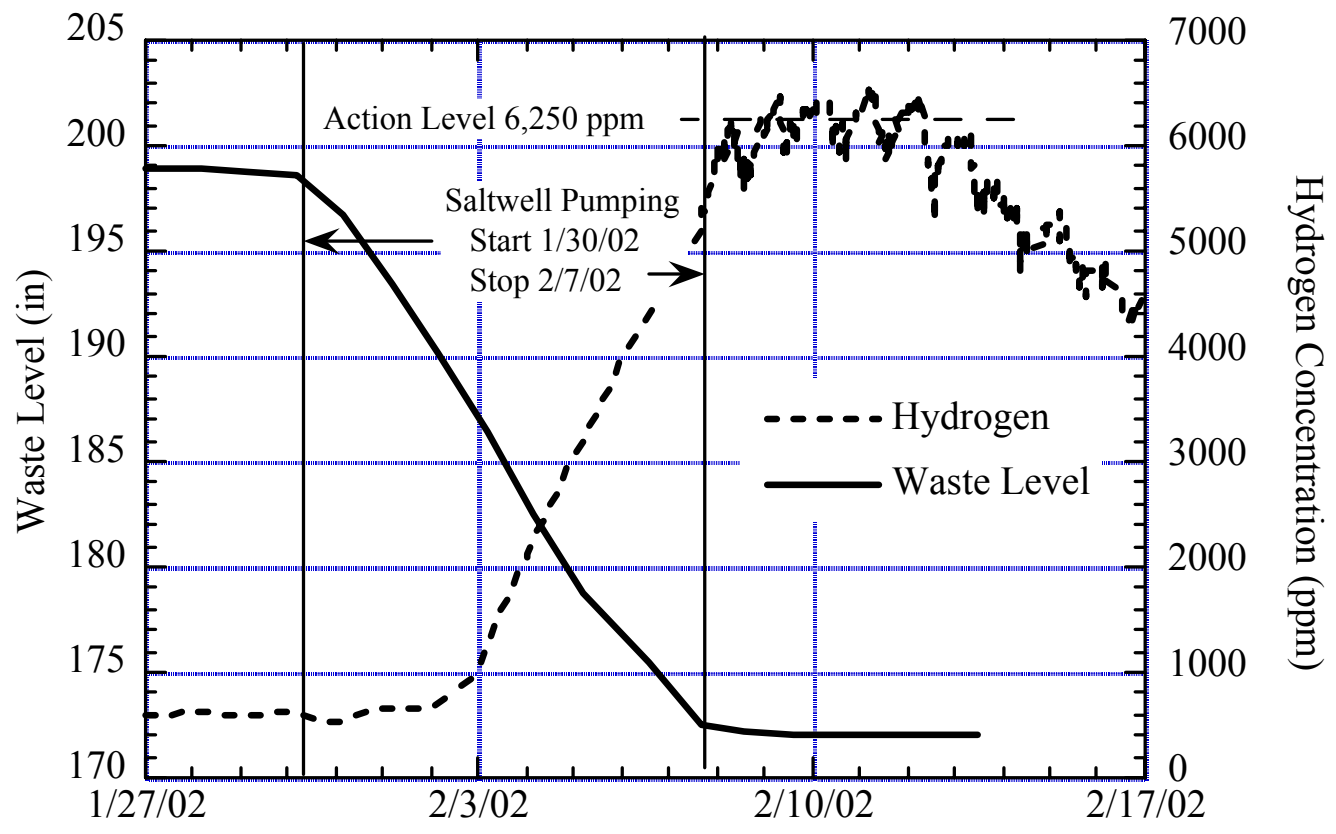

Figure 3.9. Hydrogen Concentration and Waste Level History in S-111

Saltwell pumping gas release rates are quite low but can become an issue in SSTs because the low passive ventilation rates can allow released gas to accumulate in the headspace. However, hydrogen concentrations recorded in several tanks during pumping have been well below $25 \%$ of the LFL. Gas releases during saltwell pumping can be controlled, with some delay, by shutting down the pump (Watrous et al. 2000). This ability to control gas releases is also demonstrated by the behavior in S-111 after pumping was shut down (Figure 3.9).

During saltwell pumping, headspace sampling is required periodically (e.g., weekly) to detect whether the hydrogen concentration is approaching the action level (Watrous et al. 2000). This low frequency is consistent with very low gas release rates, but monitoring is needed because the potentially low ventilation rates allow gas to accumulate. Recent experience with S-111 was a good test of this requirement because it already had continuous gas monitoring. Applying a weekly schedule to the hydrogen concentration transient in Figure 3.9 and assuming the first measurement was made when pumping began January 30, a measurement would have been made on February 6, when the concentration was over $4,000 \mathrm{ppm}$. If this relatively high reading did not trigger more frequent monitoring, a third measurement would have occurred February 13. Extrapolating the slope of the hydrogen concentration curve from February 6 indicates it could have reached as high as $10,000 \mathrm{ppm}$ at the third sample. This implies that strict weekly 
monitoring would not have prevented exceeding the action level but would have permitted pumping to shut down well before reaching the LFL.

\subsubsection{Potential Changes in Waste Classification After Saltwell Pumping}

While saltwell pumping produces a marked change in the waste configuration of SSTs, its main effects are to release up to about half of the gas inventory in a tank and to reduce the amount of liquid-saturated waste in which new gas can be generated and stored. The absence of supernatant and the presence of a high lithostatic load also prevent any possibility of a BDGRE. At the same time, removal of supernatant and subsidence of the drained sediment increases the headspace, providing more dilution volume for future releases. All these changes would tend to move a tank's classification toward Waste Group C. Therefore, while reevaluation of the tank classification may be operationally beneficial, it is not required for saltwell pumping.

\subsubsection{Potential Changes in Hydrogen Generation After Saltwell Pumping}

Saltwell pumping reduces the potential for steady-state flammability by removing liquid waste from the tank and increasing the headspace. Therefore, the steady-state gas generation rate is bounded by the prepumping calculation and need not be reevaluated.

\subsection{Saltcake Dissolution}

Saltcake dissolution is a proposed method of waste retrieval from SSTs containing saltcake (Estey et al. 2001). Water is sprayed on the waste to dissolve the soluble salts, and the resulting brine is pumped out of the tank and transferred to a DST at about the same rate that it is created. Though the water spray and pumping system may also remove insoluble solids, the primary product delivered to the receiving DST is a concentrated liquid. Saltcake dissolution does not include small water additions associated with line flushes, lancing in instruments or saltwell screens, and the like, which are covered in Section 3.5. The addition of brine to the receiving DST is covered under Waste Addition in Section 3.2.

Batch transfer followed by dissolution was used to dissolve the thick crust layer and remediate gas retention in SY-101 (Johnson et al. 2000). A similar batch process is also planned as a step in delivering waste feed from the DSTs to the vitrification plant. A large volume of water would be added to the tank following removal of most of the existing supernatant. After dissolution, which may be aided by mixing, the brine would be transferred to a staging tank. This operation is not authorized and is not yet described in sufficient detail to permit analysis.

The global waste disturbance of saltcake dissolution is the destruction of the waste microstructure by dissolution of solid particles and the major reduction in waste volume.

\subsubsection{Mechanisms for Gas Release During Saltcake Dissolution}

Stewart (2001) studied gas release during saltcake dissolution. Any gas retained in the region of the waste in which soluble solids are dissolved is assumed to be released. Waste in 
tanks with mostly insoluble waste would not be disturbed by water addition, and little gas would be released. Because the solvent fluid is less dense than the saturated interstitial brine, the rate of dissolution and therefore the rate of gas release are limited by the rate at which the brine can drain from the waste so the solvent can contact the solids. The gas release rate thus depends on the brine pumping rate and is not accelerated by adding excess water above that which can contact the waste. If the solid matrix around the bubbles is not liquid saturated, pathways exist to the tank headspace and the gas generated in the waste is dissipated rapidly by diffusion (Stewart et al. 1996). Therefore, potentially hazardous gas releases can only occur by disrupting liquid-saturated solids.

Ammonia evaporation is not a factor during saltcake dissolution. Dilution accompanying dissolution reduces the ammonia evaporation rate significantly because the solubility of ammonia increases greatly as the $\mathrm{pH}$ decreases with dilution. A water spray is also a very effective way to scavenge ammonia vapor from the headspace. This was clearly demonstrated during back-dilution in SY-101, where the headspace ammonia concentration decreased from around $400 \mathrm{ppm}$ to less than $100 \mathrm{ppm}$ in a matter of minutes after back-dilution began (Mahoney et al. 2000). Spraying water on the waste surface for retrieval inhibits ammonia release and probably reduces ammonia concentration below the historic baseline values.

\subsubsection{Expected Gas Releases During Saltcake Dissolution}

Based on the fact that dissolution and therefore gas release during dissolution are limited by the rate at which brine drains from the waste, a bounding gas release can be computed given the brine pumping rate. Water is assumed to be added at a rate $\mathrm{Q}_{\mathrm{wIN}}$ such that the resulting brine production rate is equal to the brine capacity of the saltwell pump, Qвоuт. Assuming that dissolution immediately releases all the gas stored in the waste being dissolved, the gas release rate, $\mathrm{Q}_{\mathrm{GAS}}$, is the product of the gas volume fraction, $\alpha$, and the dissolution rate as follows:

$$
\mathrm{Q}_{\mathrm{GAS}}=\alpha\left[\mathrm{F}_{\mathrm{DSLN}} / \mathrm{F}_{\mathrm{BRINE}}\right] \mathrm{Q}_{\text {BOUT }}
$$

where $\mathrm{F}_{\mathrm{DSLN}}$ is the volume of original waste in which soluble solids are dissolved per unit volume of water added, and $\mathrm{F}_{\mathrm{BRINE}}$ is the volume of total brine produced (water added plus dissolved solids plus interstitial liquid liberated) per unit volume of water added.

The resulting release rates from typical tanks are quite low, especially when the brine is removed by the saltwell pumping system (see Section 3.3). For example, the solubility of U-107 waste is such that $\mathrm{F}_{\mathrm{DSLN}}=1.8$ and $\mathrm{F}_{\mathrm{BRINE}}=2.15$, and the gas volume fraction averages 0.17 at the $95 \%$ confidence level, as estimated with the BPE calculation and neutron log integration (Hedengren et al. 2001). The U-107 proof-of-concept demonstration is planned to apply a maximum of 2,400 gallons of water per day during one eight-hour shift while the saltwell pump operates continuously. Assuming all of it dissolves waste, the water will create $\mathrm{F}_{\mathrm{BRINE}}$ times its volume in brine, which requires an average pumping rate of 5,160 gal-brine/day, or $3.58 \mathrm{gpm}$. Eq. (3.4.1) indicates a corresponding gas release of $879 \mathrm{gal} / \mathrm{day}$ or $117 \mathrm{scfd}$. If the whole tank is to be retrieved, this release rate should continue until essentially the entire gas inventory is released. 
The self-limiting behavior of gas release by dissolution makes the process controllable. Though some lag may be expected, the dissolution rate and gas release rate can theoretically be reduced relatively quickly by terminating pumping (accumulating brine forms a barrier to fresh solvent) and by shutting off the water spray (terminating the supply of fresh solvent). The most effective control is achieved if dissolution is local and the inventory of excess solvent is kept to a minimum. Though the actual response time of the gas release to changes in pumping and water addition rates are not known, saltwell pumping experience (see Section 3.3.2) indicates that it would be reasonable to expect gas release to lag cessation of pumping and spraying by about a day. However, the more local the dissolution, the shorter the lag time. These limitations indicate that saltcake dissolution should not produce much larger gas releases than those expected during saltwell pumping without concurrent dissolution.

The gas releases and waste behavior during the dissolution of the crust layer in SY-101 qualitatively validated our understanding of the physics of solvent flow and saltcake dissolution. The initial 120-inch crust layer in this tank was comparable to the non-supernatant waste in U107 in thickness but contained almost twice the gas. The crust was dissolved in a series of three waste transfers and back-dilutions that eventually added 525,000 gallons of water. The bulk of the crust dissolution and gas release occurred in the second back-dilution, when the headspace hydrogen concentration peaked at about $3,000 \mathrm{ppm}$. This concentration represents a sudden release of about $200 \mathrm{scf}$ of hydrogen, much more than can be expected during dissolution of SST waste.

\subsubsection{Gas Monitoring Considerations During Saltcake Dissolution}

The potential hazard for saltcake dissolution is not the gas release rate so much as the low passive ventilation rates that allow the gas to accumulate. The headspace hydrogen concentration at a given time, t, during saltcake dissolution can be computed by (Stewart 2001):

$$
\mathrm{C}_{\mathrm{H}}(\mathrm{t})=\frac{\mathrm{Q}_{\mathrm{GAS}} \chi_{\mathrm{H}}}{\mathrm{Q}_{\mathrm{A}}}+\left(\mathrm{C}_{0}-\frac{\mathrm{Q}_{\mathrm{GAS}} \chi_{\mathrm{H}}}{\mathrm{Q}_{\mathrm{A}}}\right)\left(1-\frac{\mathrm{Q}_{\mathrm{B}}}{\mathrm{V}_{\mathrm{HS} 0}} \mathrm{t}\right)^{\frac{\mathrm{Q}_{\mathrm{A}}}{\mathrm{Q}_{\mathrm{B}}}}
$$

where

$\mathrm{C}_{0} \quad=$ initial headspace hydrogen concentration

$\mathrm{V}_{\mathrm{HS} 0}=$ initial headspace volume

and

$\chi_{\mathrm{H}}=$ hydrogen fraction of the retained gas in the waste

where

$$
\begin{aligned}
& \mathrm{Q}_{\mathrm{A}}=\mathrm{Q}_{\mathrm{VIN}}+\mathrm{Q}_{\mathrm{GAS}} \\
& \mathrm{Q}_{\mathrm{B}}=\mathrm{Q}_{\mathrm{WIN}}-\mathrm{Q}_{\mathrm{BOUT}}-\mathrm{Q}_{\mathrm{GAS}} \frac{\mathrm{P}_{\mathrm{A}}}{\mathrm{P}_{\mathrm{S}}}
\end{aligned}
$$

$\mathrm{P}_{\mathrm{A}}=$ pressure in the headspace atmosphere $(\sim 1 \mathrm{~atm})$

$\mathrm{P}_{\mathrm{S}}=$ pressure at which the gas is retained in the sediment

$\mathrm{Q}_{\mathrm{GAS}}=$ gas release rate defined by Eq. (3.4.1)

$\mathrm{Q}_{\mathrm{VIN}}=$ estimated headspace passive (or measured active) ventilation rate

$\mathrm{Q}_{\mathrm{WIN}}=$ solvent water flow rate 


\section{$\mathrm{Q}_{\text {BOUT }}=$ brine pumping rate.}

Figure 3.10 shows the headspace hydrogen concentration as a function of time obtained by applying Eq. (3.4.2) to the U-107 proof-of-concept example with higher brine pumping rates as a parameter, assuming the released gas is $50 \%$ hydrogen and the headspace ventilation rate is $2.5 \mathrm{cfm}$. The figure shows that the hydrogen concentration increases monotonically, even for the lowest pumping rates. The rate of rise is proportional to the pumping rate. At a 10-gpm pumping rate, it takes about six days to reach $25 \%$ of the LFL and almost 30 days at $3 \mathrm{gpm}$. However, at higher pumping rates, hydrogen concentration rises rapidly. At 100 gpm it takes 12 hours to reach $25 \%$ LFL and at 300 gpm less than 2 hours.

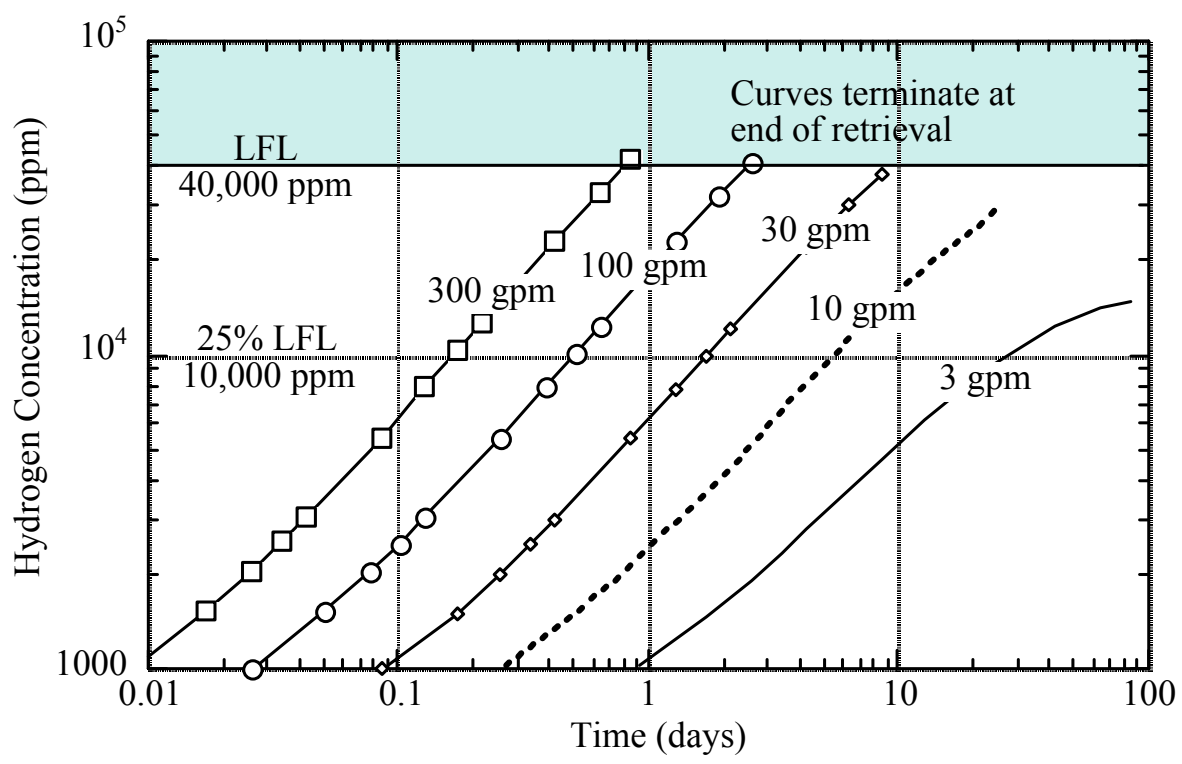

Figure 3.10. Hydrogen Concentration Versus Time for Saltcake Dissolution; Group B Tank

Because gas release during saltcake dissolution depends on having the brine continually removed, the associated gas release can be halted after some lag time by shutting down the pump and water spray system. To ensure that the system can be shut down well before reaching the LFL, the headspace hydrogen concentration must be monitored at an adequate frequency. If it is desired to shut down at $25 \%$ of the LFL, for example, the period between headspace samples should be no more than half the estimated time to reach $25 \%$ of the LFL. The required gas monitoring frequency must therefore by at least $2 / \mathrm{t}$ samples per unit time. The time required to reach a given hydrogen concentration, $\mathrm{C}_{\mathrm{H}}$, during saltcake dissolution can be calculated by solving Eq. (3.4.2) for $t$ to give

$$
\mathrm{t}=\frac{\mathrm{V}_{\mathrm{HS} 0}}{\mathrm{Q}_{\mathrm{B}}}\left[1-\left(\frac{\mathrm{C}_{\mathrm{H}}-\frac{\mathrm{Q}_{\mathrm{GAS}} \chi_{\mathrm{H}}}{\mathrm{Q}_{\mathrm{A}}}}{\mathrm{C}_{0}-\frac{\mathrm{Q}_{\mathrm{GAS}} \chi_{\mathrm{H}}}{\mathrm{Q}_{\mathrm{A}}}}\right)^{\frac{\mathrm{Q}_{\mathrm{B}}}{\mathrm{Q}_{\mathrm{A}}}}\right]
$$


For the saltcake dissolution example described above, the required gas monitoring frequencies defined as $\mathrm{f}=2 / \mathrm{t}$, with $\mathrm{t}$ defined by Eq. (3.4.3) for an action level of 10,000 ppm hydrogen, are plotted as a function of pumping and ventilation rates in Figure 3.11. The figure shows that the weekly monitoring required for saltwell pumping is adequate for the initial saltcake dissolution retrieval demonstration operations. With higher pumping rates, daily monitoring may be necessary.

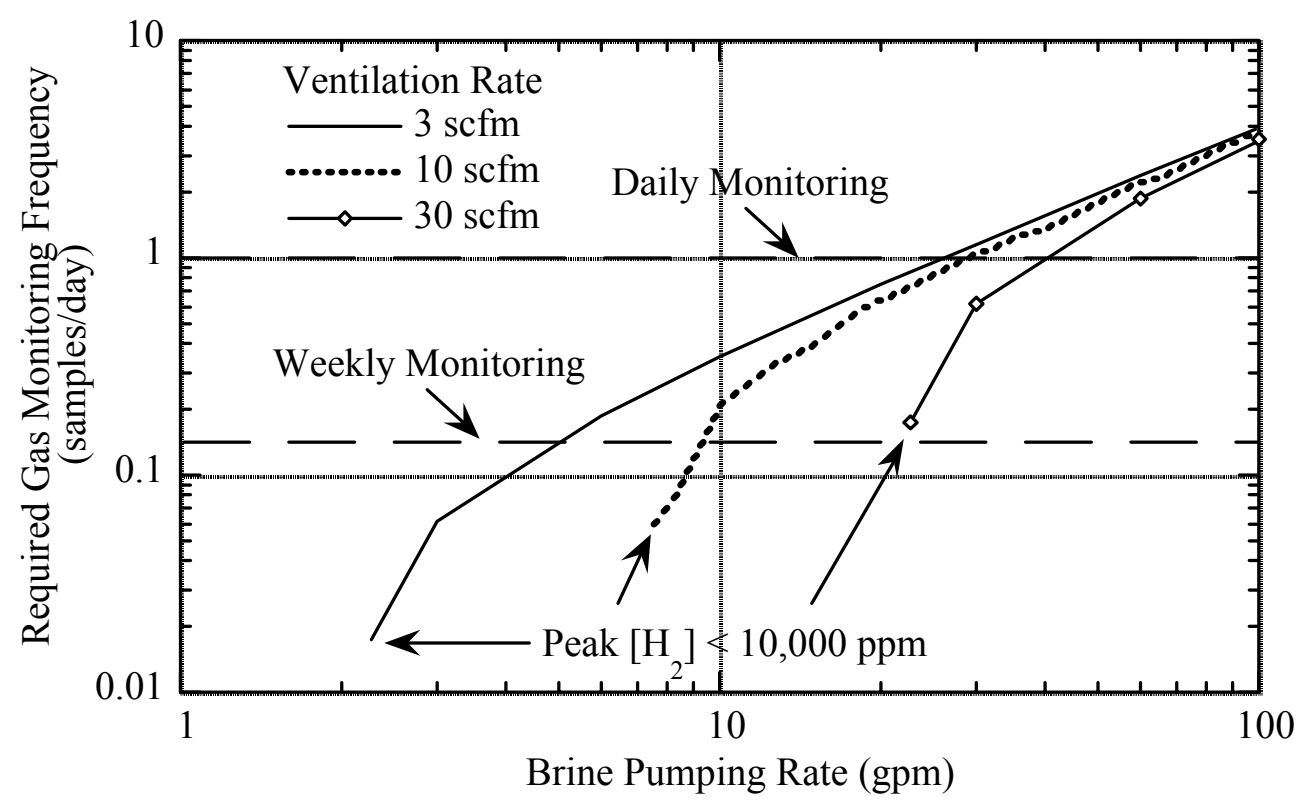

Figure 3.11. Required Gas Monitoring Frequency Versus Brine Pumping Rate

\subsubsection{Potential Changes in Waste Classification After Saltcake Dissolution}

All SSTs that are successfully retrieved will move to Waste Group C because little waste will be left to generate and retain gas. Saltcake dissolution increases the headspace and reduces the consequences of gas releases even if not carried all the way to retrieval. Therefore, because the process cannot adversely affect a tank's gas retention and release behavior, no reevaluation of waste group is necessary.

\subsubsection{Potential Changes in Hydrogen Generation After Saltcake Dissolution}

Because saltcake dissolution removes waste from a tank, the post-retrieval hydrogen generation rate will be less than the initial rate. Therefore, the steady-state flammability status is bounded by the initial calculation, and no reevaluation is necessary.

\subsection{Water Addition}

Adding small volumes of water to both DSTs and SSTs is necessary from time to time to flush transfer lines, lance instruments into the waste, decontaminate hardware removed from the 
waste, or install retrieval systems. Larger water additions are possible. The global waste disturbance from water addition occurs via an increase in hydrostatic pressure and potentially by dissolution of soluble solids. Some water additions such as lancing may also be a local waste disturbance that releases a small additional gas volume. Larger water additions can decrease the tank headspace, which adversely affects gas releases and may change the tank classification. Large water additions with concurrent or subsequent brine removal should be treated as SST saltcake dissolution, as described in Section 3.4.

\subsubsection{Mechanisms for Gas Release During Water Addition}

The dominant mechanism for gas release by water addition is dissolution of solids retaining gas. Gas release due to water addition is an issue only in tanks with waste consisting mainly of saltcake. In saltcake SSTs, water added to the waste gradually invades interstitial liquid via diffusion and convection. As it contacts soluble salts, the water will eventually dissolve waste that retains gas. The same gas release mechanism as saltcake dissolution would prevail but would act much slower because brine is not removed to allow water to contact unsaturated waste.

In saltcake DSTs the added water would first mix with the lighter, more dilute supernatant near the surface. This would tend to dissolve the floating crust layer, if present, releasing the gas contained therein in a manner similar to but in much smaller quantity than SY-101 surface level rise remediation. ${ }^{\text {(a) }}$ If dissolving the crust does not saturate the liquid, convection would gradually mix the dilute liquid throughout the supernatant, where it would slowly penetrate the sediment layer, dissolve additional solids, and release gas as it does in an SST.

Because water is less dense than supernatant in any DST, adding water lowers the average supernatant density and raises the neutral buoyancy gas fraction. This and the consequent compression of retained gas would significantly reduce the likelihood of BDGREs during the addition, even in Waste Group A tanks.

\subsubsection{Expected Gas Releases During Water Addition}

As seen in the analysis of saltcake dissolution in SSTs in Section 3.4 or in saltcake DSTs with a significant crust, gas release resulting from dissolution by water addition would be very slow and would be a concern only for passively ventilated tanks. A maximum water addition can be calculated as the volume required to raise the headspace hydrogen concentration to the LFL $\left(\left[\mathrm{H}_{2}\right]_{\mathrm{LFL}}=4 \mathrm{vol} \%\right)$ with zero ventilation. These very conservative assumptions are applied:

- Ventilation rate is zero

- The tank is full with a headspace volume $\left(\mathrm{V}_{\mathrm{HS}}\right)$ of 30,000 cubic feet

- Waste in which soluble solids are dissolved and gas is released is twice the volume of water added ( 2 gal affected per gal of water added, $\mathrm{F}_{\mathrm{DSLN}}=2$ )

- Retained gas volume fraction $\left(\alpha_{\text {avg }}\right)$ is 0.2

- Retained gas is $100 \%$ hydrogen $\left(\mathrm{X}_{\mathrm{H}}=1\right)$.

(a) Crust layers in DSTs are less than $1 \mathrm{~m}$ thick; SY-101's crust before remediation was about $3 \mathrm{~m}$ thick. 
The minimum volume of water added to a saltcake tank that would release sufficient gas to bring the headspace to the LFL with zero ventilation can be expressed as

$$
\mathrm{V}_{\max }=\frac{\mathrm{V}_{\mathrm{HS}}\left[\mathrm{H}_{2}\right]_{\mathrm{LFL}}}{\alpha_{\mathrm{avg}} \mathrm{X}_{\mathrm{H}} \mathrm{F}_{\mathrm{DSLN}}}
$$

Evaluating Eq. (3.5.1) for the conditions stated above,

$$
\begin{aligned}
& \mathrm{V}_{\text {max }}=\frac{30,000\left(\mathrm{ft}^{3} \mathrm{HS}\right) \cdot 0.04\left(\mathrm{ft}^{3} \mathrm{H}_{2} \text { at LFL } / \mathrm{ft}^{3} \mathrm{HS}\right)}{0.2\left(\mathrm{ft}^{3} \text { gas } / \mathrm{ft}^{3} \text { waste }\right) \cdot 1.0\left(\mathrm{ft}^{3} \mathrm{H}_{2} / \mathrm{ft}^{3} \text { gas }\right) \cdot 2\left(\mathrm{ft}^{3} \text { waste } / \mathrm{ft}^{3} \text { water }\right)} \cdot 7.47\left(\mathrm{gal} / \mathrm{ft}^{3}\right) \\
& \mathrm{V}_{\text {max }}=22,410 \text { gallons of water. }
\end{aligned}
$$

Batch water additions greater than this upper-bound threshold should be treated as saltcake dissolution under Section 3.4. Eq. (3.5.1) can be applied with data on specific tanks to arrive at much larger threshold volumes. This calculation would also apply to crust-containing DSTs with inoperative ventilation. Gas release due to dissolution is of no concern in actively ventilated tanks.

\subsubsection{Gas Monitoring Considerations During Water Addition}

Water additions less than the upper-bound volume in a passively ventilated tank or any water addition in an actively ventilated tank should not require monitoring. Additions greater than the threshold volume in SSTs with passive ventilation should be treated as saltcake dissolution with the monitoring frequency defined by Eq. (3.4.2).

\subsubsection{Potential Changes in Waste Classification After Water Addition}

Water addition decreases the headspace in a tank and could potentially move a tank from Group C into Group B, or Group A if it were already exhibiting spontaneous BDGREs, and requires a waste group evaluation. In the initial calculations by Barker and Hedengren (2002), potential changes in waste group classification from hypothetical additions of 10,000 gallons of water and caustic have been evaluated for all tanks. This small-volume addition caused no changes in tank classification. Because water is less dense than the supernatant in all tanks, water additions always reduce the mixed supernatant density and increase the neutral buoyancy gas fraction without affecting the gas generation rate. Thus it is not possible for water addition to cause a tank to begin spontaneous BDGREs and move from Waste Group B to A.

In SSTs, however, a large water addition could create a supernatant layer sufficiently deep to make the energy ratio defined by Eq. (2.18) exceed the threshold value. In DSTs, this would require a Group B tank to be evaluated for BDGRE potential. However, the buoyancy ratio criterion (see Section 2.3.2.1) cannot be applied to SSTs because their waste properties and configuration differ considerably from those on which the model is based. To avoid this situation, water additions to Group B SSTs should be limited such that the energy ratio defined 
by Eq. (2.18) remains less than the threshold. Solids dissolution should be estimated in this calculation to account for all the liquid available.

By decreasing the headspace, raising the neutral buoyancy gas fraction, and increasing the hydrostatic pressure on the retained gas, water additions raise the peak hydrogen concentration from ongoing spontaneous BDGREs in Group A (DST) tanks. Though water also dissolves solids and thus tends to reduce BDGRE potential and size, the former effects dominate unless a very large volume of water is used. ${ }^{\text {(a) }}$ However, the increase in peak hydrogen concentration resulting from spontaneous BDGREs after a water addition can be estimated easily. The headspace hydrogen concentration, $\mathrm{C}_{\mathrm{H}}$, following a BDGRE can be conservatively calculated from the gas release volume, $V_{R}$, the fraction of hydrogen in the released gas, $\chi_{\mathrm{H}}$, the headspace volume, $\mathrm{V}_{\mathrm{HS}}$, and the initial concentration, $\mathrm{C}_{0}$, assuming an instantaneous release (Meyer and Wells 2000):

$$
\mathrm{C}_{\mathrm{H}}=\chi_{\mathrm{H}} \frac{\mathrm{V}_{\mathrm{R}}}{\mathrm{V}_{\mathrm{HS}}}+\mathrm{C}_{0}
$$

For a given gob volume, $\mathrm{V}_{0}$, in the sediment (see Section 2.3.2), the volume of gas released into the headspace when the gob goes buoyant can be expressed as

$$
\mathrm{V}_{\mathrm{R}}=\alpha_{\mathrm{NB}} \Phi \frac{\mathrm{P}_{\mathrm{S}}}{\mathrm{P}_{\mathrm{A}}} \mathrm{V}_{0}
$$

where

$$
\begin{aligned}
& \alpha_{\mathrm{NB}}=\text { neutral buoyancy gas fraction } \\
& \Phi=\text { gas release fraction } \\
& \mathrm{P}_{\mathrm{S}}=\text { hydrostatic pressure on the gas in the sediment layer } \\
& \mathrm{P}_{\mathrm{A}}=\text { ambient pressure in the tank headspace. }
\end{aligned}
$$

The neutral buoyancy model for the gas release fraction states that gas is released until the gob returns to neutral buoyancy at the waste surface. This results in the expression

$$
\Phi=1-\frac{\mathrm{P}_{\mathrm{A}}}{\mathrm{P}_{\mathrm{S}}}
$$

Combining Eq. (3.5.2), (3.5.3), and (3.5.4) and ignoring the low initial hydrogen concentration gives the overall expression for the post-GRE hydrogen concentration:

$$
\mathrm{C}_{\mathrm{H}}=\alpha_{\mathrm{NB}} \chi_{\mathrm{H}}\left(\frac{\mathrm{P}_{\mathrm{S}}}{\mathrm{P}_{\mathrm{A}}}-1\right) \frac{\mathrm{V}_{0}}{\mathrm{~V}_{\mathrm{HS}}}
$$

(a) A calculation of the water dilution volume required to dissolve sufficient solids to remediate SY-101 and the results of this operation are summarized by Johnson et al. (2000). 
Assuming that the hydrogen fraction and gob volume do not change after water addition, the peak hydrogen concentration due to a spontaneous BDGRE after water addition is related to the historic value by the ratio for the post- and pre-addition states using Eq. (3.5.5); that is,

$$
\mathrm{C}_{\mathrm{H} 2}=\mathrm{C}_{\mathrm{H} 1} \frac{\alpha_{\mathrm{NB} 2}}{\alpha_{\mathrm{NB} 1}} \frac{\left(\frac{\mathrm{P}_{\mathrm{S} 2}}{\mathrm{P}_{\mathrm{A}}}-1\right)}{\left(\frac{\mathrm{P}_{\mathrm{S} 1}}{\mathrm{P}_{\mathrm{A}}}-1\right)} \frac{\mathrm{V}_{\mathrm{HS} 1}}{\mathrm{~V}_{\mathrm{HS} 2}}
$$

Table 3.4 shows the results of applying Eq. (3.5.6) to five DSTs that experience spontaneous BDGREs. The table lists the maximum headspace hydrogen concentration for any BDGRE recorded on an SHMS and the estimated concentration had the tank been filled to 422 inches by adding water at a density of $1,000 \mathrm{~kg} / \mathrm{m}^{3}$ with no dissolution or adding caustic (discussed in Section 3.8 ) at a density of $1,500 \mathrm{~kg} / \mathrm{m}^{3}$. It is hypothetically possible to approach the LFL in SY-103 only after adding about 380,000 gallons of water. However, because it has been calculated that about 250,000 gallons of water would dissolve enough solids to prevent BDGREs altogether in SY-103, this result is plausible only under the conservative assumption of no dissolution. ${ }^{(a)}$

Table 3.4. Peak Hydrogen Concentrations After Water and Caustic Addition

\begin{tabular}{||l|c|c|c|c|c||}
\hline \multicolumn{1}{|c|}{ Event } & AN-103 & AN-104 & AN-105 & AW-101 & SY-103 \\
\hline $\begin{array}{l}\text { Maximum historic BDGRE }\left[\mathrm{H}_{2}\right] \\
(\mathrm{ppm})\end{array}$ & 1,600 & 6,100 & 17,000 & 6,200 & 5,100 \\
\hline Volume added for 422 in. (gal) & 203,000 & 108,000 & 33,000 & 35,000 & 380,000 \\
\hline Peak $\left[\mathrm{H}_{2}\right]$ after water addition $(\mathrm{ppm})$ & 5,000 & 14,000 & 23,000 & 8,400 & 49,000 \\
\hline Peak $\left[\mathrm{H}_{2}\right]$ after caustic addition $(\mathrm{ppm})$ & 3,500 & 9,200 & 20,000 & 7,100 & 19,000 \\
\hline
\end{tabular}

Further, as shown in Section 3.2.4, the addition of liquid at a lower specific gravity than the existing supernatant will reduce the buoyancy ratio. For water addition to SY-103 with no dissolution, the buoyancy ratio drops below unity with approximately 220,000 gallons added. AN-103 is a special case (it is the BDGRE tank used to set the buoyancy ratio coefficient, as described in subsection 2.3.2.1), and any water addition with no dissolution is sufficient to reduce the buoyancy ratio to below unity. It is not possible, however, to determine whether 10,000 gallons of water would indeed prevent BDGREs though some larger volume would clearly do so.

Adding water increases the peak hydrogen concentration more than adding caustic in all tanks. We conclude that, though adding water exacerbates the consequences of BDGREs in Group A tanks, the total effect is not enough to approach the LFL based on historical behavior and, in sufficient quantities, may mitigate the tank.

(a) Stewart CW and BE Wells. September 2000. A Strategy for Remediation and Return to Service for Tank 241-SY-103. Letter Report TWS01.01, Pacific Northwest National Laboratory, Richland, WA. 


\subsubsection{Potential Changes in Hydrogen Generation After Water Addition}

Water additions tend to dilute the hydrogen-generating liquid in the waste and reduce the generation rate. However, dissolution of soluble solids tends to reverse dilution and maintain the generation rate. Analysis shows that the balance of these effects favors a reduction, and the steady-state flammability potential after water addition is bounded by the existing calculation.

\subsection{Mixer Pump Operation}

Mixer pump operation is capable of producing the largest gas releases of all the activities discussed in this report. Mixing the waste can release its entire gas inventory and make the headspace flammable in Group A and B tanks. Because of this, the initial period of mixing must be planned specifically to limit the volume of waste disturbed and control the rate of gas release. Only after this degassing period can the mixer pumps be operated to their design performance. The rest of this section is devoted mainly to the degassing period.

Mixer pumps are planned to be installed as part of DST waste retrieval and staging to the vitrification plant. A mixer pump degassed the waste in SY-101 in 1993, and a pair of powerful pumps were operated more recently in AZ-101. Mixer pump operation is specific to DSTs.

The global waste disturbance of a mixer pump is the hydraulic erosion and suspension of previously settled sediment by the pump jets. The rate of disturbance depends on the pump power, jet diameter and configuration, and the duration of the operation. Thus a single run of a small pump for a short time might be classified a local waste disturbance. But mixer pump operation is considered a global disturbance because it is designed to generally mix the tank.

\subsubsection{Mechanisms for Gas Releases During Mixer Pump Operation}

Gas release during mixer pump operation is caused mainly by the disruption of the waste structure retaining the gas bubbles. However, a major secondary release mechanism is the increase in effective liquid density by suspension of solid particles. This reduces the neutral buoyancy gas fraction, which may induce BDGREs. Planning a degassing campaign thus involves limiting the amount of waste disturbed in each pump run and including the effects of induced BDGREs. After degassing is completed, periodic mixing will continue to release gas that has accumulated between pump runs by detaching bubbles from sediment particles on which they nucleated and grew.

If a liquid waste surface with a high concentration of dissolved ammonia is stirred or agitated, the evaporative mass-transfer rate of ammonia can be greatly increased. While mixing generally stirs the waste, it does not tend to produce significant ammonia evaporation. Most of the mixing action affects the region of the waste near the tank bottom and does not appear to stir the waste surface enough to boost evaporation very much (Mahoney and Stewart 2002). Headspace ammonia concentrations in SY-101 remained below 200 ppm during the intense mixing period in March 2000 and increased only slightly during pump runs (Mahoney et al. 2000). The two 300-hp mixer pumps planned for DST waste feed delivery potentially can disturb waste at four times the rate of the single 150-hp pump in SY-101, with a correspondingly 
greater potential for increased ammonia evaporation. Nevertheless, ammonia releases remain more a toxicological concern than a flammability hazard.

\subsubsection{Expected Gas Releases During Mixer Pump Operation}

The rate and amount of gas released depends on the rate and volume of waste disturbed as well as the gas volume fraction retained. It is theoretically possible for multiple large mixer pumps to release enough gas to bring the headspace hydrogen concentration to the LFL if they were started up and run at full power in Waste Group A or B tanks. ${ }^{\text {(a) }}$ A specific degassing plan is needed in these tanks that disturbs the waste a little at a time until most of the retained gas has been released.

After degassing is complete, however, periodic mixing can only release gas that has accumulated between pump runs. This is of no concern unless the pump has been idle for a very long time. Table 3.5 shows the time required for the tanks currently experiencing BDGREs to accumulate enough gas to make the headspace flammable (if possible) and to achieve neutral buoyancy. This assumes that mixing is terminated after the tank is initially completely degassed while the volumetric gas generation Barker and Hedengren (2002) calculated for tank classification is $100 \%$ retained in the sediment. The table shows that AN-104 and AN-105 would return to Waste Group B in 1.3 and 1.6 years, respectively, while the first BDGRE could not occur until after 7 and 4 years, respectively. AW-101 and SY-103, classified in Waste Group C, would require three years to return to BDGRE behavior.

Table 3.5. Time to Return to BDGRE Behavior After Degassing

\begin{tabular}{|l|c|c|c||}
\hline Tank & $\begin{array}{c}\text { Current Waste } \\
\text { Group }\end{array}$ & $\begin{array}{c}\text { Years to Re-Enter } \\
\text { Waste Group B }\end{array}$ & $\begin{array}{c}\text { Years for Neutral } \\
\text { Buoyancy }\end{array}$ \\
\hline AN-103 & A & 3.4 & 16 \\
\hline AN-104 & A & 1.6 & 4 \\
\hline AN-105 & A & 1.3 & 7 \\
\hline AW-101 & C & N/A & 3 \\
\hline SY-103 & C & N/A & 3 \\
\hline
\end{tabular}

In Waste Group A tanks, the sediment suspended in the supernatant by mixing increases the effective supernatant density and the neutral buoyancy gas fraction, inducing BDGREs. These induced gas releases are superimposed on the gas released as a result of mixing. Such gas releases will also be induced in Group $\mathrm{C}$ tanks that experience BDGREs, but they are of minor consequence because these tanks do not contain sufficient gas to make a well-mixed headspace flammable.

Borderline Group B tanks may also experience induced BDGREs during mixing. The analysis derived in Section 3.2 for BDGREs induced by waste addition can be applied to

(a) As described in Section 2.4.1, Waste Group A and B tanks store sufficient gas to make the headspace flammable if all of it were suddenly released; Waste Group C tanks do not. Waste Group A tanks exhibit spontaneous BDGREs, while tanks in Waste Group B do not. 
determine whether mixing will induce a BDGRE. A bounding liquid density can be calculated by assuming that the entire sediment layer is mixed with the initial supernatant as follows:

$$
\rho_{\mathrm{L} 2}=\frac{\rho_{\mathrm{L} 1} \mathrm{H}_{\mathrm{L}}+\rho_{\mathrm{S}} \mathrm{H}_{\mathrm{S}}}{\mathrm{H}_{\mathrm{L}}+\mathrm{H}_{\mathrm{S}}}
$$

where $\rho_{\mathrm{L} 1}$ is the initial supernatant density, $\rho_{\mathrm{S}}$ and $\mathrm{H}_{\mathrm{S}}$ are the sediment density and depth, respectively, and $\mathrm{H}_{\mathrm{L}}$ is the supernatant depth. This assumption about the density makes the analysis truly bounding. If the entire sediment layer is suspended in the supernatant, mixing is complete and no sediment layer remains to experience a BDGRE.

The final buoyancy ratio is calculated from the gas volume fraction and the neutral buoyancy gas fraction, including the effects of the increased density. If the final buoyancy ratio is greater than 1, a BDGRE may occur during mixing. The final gas volume fraction, accounting for compression by the increased density, is given by Eq. (3.1.2). Combining this with Eq. (2.12) for the final neutral buoyancy gas volume fraction provides an expression for the final buoyancy ratio:

$$
\mathrm{BR}_{2}=\frac{\alpha_{2}}{\alpha_{\mathrm{NB} 2}}=\frac{\alpha_{1} \rho_{\mathrm{S}} /\left(\rho_{\mathrm{S}}-\rho_{\mathrm{L} 2}\right)}{\alpha_{1}+\left(1-\alpha_{1}\right) \mathrm{P}_{2} / \mathrm{P}_{1}}
$$

where the pressure ratio is calculated by Eq. (3.2.4) using the new liquid density from Eq. (3.6.1) and a constant liquid depth. Substituting the product of the initial buoyancy ratio (Eq. 2.13) and the initial neutral buoyancy gas fraction (Eq. 2.12) for the initial gas fraction yields

$$
\mathrm{BR}_{2}=\frac{\rho_{\mathrm{S}} /\left(\rho_{\mathrm{S}}-\rho_{\mathrm{L} 2}\right)}{1+\left[\frac{\rho_{\mathrm{S}}}{\mathrm{BR}_{1}\left(\rho_{\mathrm{S}}-\rho_{\mathrm{L} 2}\right)}-1\right] \mathrm{P}_{2} / \mathrm{P}_{1}}
$$

Applying Eq. (3.6.3) to the current Group B DSTs implies that AN-107 could experience an induced BDGRE during mixing. While very conservative, this result suggests that induced gas releases larger than those caused by the waste disturbance alone are possible in some Group B tanks. Though BDGREs can also be induced in some Group $\mathrm{C}$ tanks, they are not of concern because these tanks contain insufficient gas to reach flammability.

Wells et al. (2002) performed detailed analyses of these effects for DSTs AN-103, AN-104, AN-105, and AW-101. The analyses were based on a disturbance of a specified fraction of the sediment each pump run with one run every 8 or 24 hours, continuing until the entire sediment layer was mixed. Gas releases from induced BDGREs are also included via Monte Carlo simulation using essentially the same model described for waste removal in Section 3.1. The base case assumes that $5 \%$ of the sediment volume is disturbed every 24 hours. Other combinations were a $5 \%$ disturbance every 8 hours to investigate the sensitivity to pump schedule and a $20 \%$ disturbance every 8 or 24 hours to test the effect of increasing disturbance. The headspace ventilation rate was $120 \mathrm{scfm}$ in AW-101 and $100 \mathrm{scfm}$ in the other tanks. 
The results are summarized in Table 3.6, which lists peak hydrogen concentrations predicted during the mixing process and the fraction of gas that was released by induced BDGREs. The results are consistent with the supernatant decant analyses. If the volume of waste disturbed per mixer pump run is limited to no more than $5 \%$ of the sediment every 24 hours, the peak hydrogen concentrations remain well below the LFL, including the effect of induced BDGREs.

Table 3.6. Summary of Mixing Gas Release Analysis Results

\begin{tabular}{|c|c|c|c|c|}
\hline Tank & Run & Quantity & Median & $95 \%$ CL \\
\hline \multirow[t]{6}{*}{ AN-105 } & $5 \%$ every 24 hours & Peak hydrogen concentration (ppm) & 7,500 & 15,100 \\
\hline & & Fraction gas release by BDGREs & 0.09 & 0.23 \\
\hline & $20 \%$ every 24 hours & Peak hydrogen concentration (ppm) & 20,300 & 28,400 \\
\hline & & Fraction gas release by BDGREs & 0.08 & 0.22 \\
\hline & $5 \%$ every 8 hours & Peak hydrogen concentration (ppm) & 9,800 & 17,100 \\
\hline & & Fraction gas release by BDGREs & 0.09 & 0.23 \\
\hline \multirow[t]{4}{*}{ AN-104 } & $5 \%$ every 24 hours & Peak hydrogen concentration (ppm) & 5,500 & 10,600 \\
\hline & & Fraction gas release by BDGREs & 0.12 & 0.26 \\
\hline & $20 \%$ every 24 hours & Peak hydrogen concentration (ppm) & 12,900 & 18,500 \\
\hline & & Fraction gas release by BDGREs & 0.10 & 0.25 \\
\hline \multirow[t]{2}{*}{ AN-103 } & $5 \%$ every 24 hours & Peak hydrogen concentration (ppm) & 15,300 & 25,800 \\
\hline & & Fraction gas release by BDGREs & 0.20 & 0.33 \\
\hline \multirow[t]{4}{*}{ AW-101 } & $5 \%$ every 24 hours & Peak hydrogen concentration (ppm) & 1,800 & 2,700 \\
\hline & & Fraction gas release by BDGREs & 0.00 & 0.14 \\
\hline & $20 \%$ every 8 hours & Peak hydrogen concentration (ppm) & 6,900 & 8,800 \\
\hline & & Fraction gas release by BDGREs & 0.00 & 0.13 \\
\hline
\end{tabular}

At the $95 \%$ confidence level, the peak hydrogen concentration during mixing was predicted to be $15,100 \mathrm{ppm}$ for $\mathrm{AN}-105$ and $10,600 \mathrm{ppm}$ for AN-104. In both of these tanks, about $25 \%$ of the gas release was due to BDGREs at the $95 \%$ confidence level. Increasing the disturbance to $20 \%$ every 24 hours raised the peak hydrogen concentration at the $95 \%$ confidence level to $28,400 \mathrm{ppm}$ and $18,500 \mathrm{ppm}$ in AN-105 and AN-104, respectively. However, the fraction of gas released by BDGREs remained at about $25 \%$ in both tanks. Increasing the frequency to a $5 \%$ disturbance every 8 hours in AN-105 did not change the result appreciably.

AW-101 showed a very low hydrogen concentration for the base case, reaching only $2,700 \mathrm{ppm}$ at the $95 \%$ confidence level with only $14 \%$ released by BDGREs. The median result showed no BDGREs at all. A more aggressive simulation was performed with $20 \%$ of the sediment disturbed every eight hours. This raised the peak hydrogen concentration to $8,800 \mathrm{ppm}$ at the $95 \%$ confidence level with $13 \%$ of the gas released by BDGREs. Using the proposed pumps, operation of one mixer pump in AW-101 at a fixed azimuth for 14 minutes creates approximately a 5\% disturbance (Wells et al. 2002).

About the same result was predicted for AN-103 with a 5\% disturbance every 24 hours and AN-105 with a $20 \%$ disturbance every 24 hours. At the $95 \%$ confidence level, peak hydrogen concentration was $25,800 \mathrm{ppm}$ with $33 \%$ of the gas resulting from induced BDGREs. The 
median for hydrogen concentration in AN-103 was 15,300 ppm; 20\% of the gas was released by BDGREs. This matches predictions at the 95\% confidence level for the same case in AN- 105 .

That none of the four tanks analyzed exceeded the LFL during degassing should not be taken to imply that no limitations are needed on mixer pump operations. The degassing schedules studied were chosen specifically as possible plans that could be accomplished safely. More aggressive mixer pump operation might, and unlimited mixer pump operation would, cause the tank headspace to become flammable. A carefully considered degassing plan is absolutely necessary in these tanks or any DST in Waste Group A or B.

\subsubsection{Gas Monitoring Considerations During Mixer Pump Operation}

The results in Table 3.6 show that, though BDGREs may be induced, most of the total gas released from a tank during mixing is produced by the action of the mixer pump. The gas release rates from both processes are high, and continuous monitoring is required to follow the hydrogen concentration transient.

No "stop-start" simulation was run for the mixer pump simulations as it was for supernatant decant. However, because there is a considerable lag (up to an hour or more) between mixer pump startup and peak gas release, a nominal mixer pump run might be completed before the hydrogen concentration rises significantly. Therefore, terminating mixer pump operation at the action level is not expected to be very effective at controlling gas release. On the other hand, delaying pump restart until after the hydrogen concentration falls back below a threshold value (500 ppm was used during mixing in SY-101) could have a measurable mitigating effect. Also, because of the delay and the potential for secondary induced BDGREs occurring even later, gas monitoring should be continued for some time after each pump run during degassing to ensure that the peak hydrogen concentration has been observed.

Active ventilation of $100 \mathrm{scfm}$ was specified for the simulations described in Section 3.6.2. Because mixing releases essentially all of the retained gas in a tank, compared with 25 to $33 \%$ released by supernatant decant (see Section 3.1.2), the peak hydrogen concentration is more sensitive to the ventilation rate. Figure 3.12 shows the effect of varying the ventilation rate from 10 to $200 \mathrm{scfm}$ in two of the Monte Carlo simulations (representing the same degassing operation but with altered input parameters) of the base mixing case in AN-103 (5\% disturbance every 24 hours) that produced a peak hydrogen concentration of $25,800 \mathrm{ppm}$ at the $95 \%$ confidence level. The curve fit through the results shows that the LFL would have been exceeded for ventilation rates less than about $20 \mathrm{scfm}$. Increasing the ventilation rate to $500 \mathrm{scfm}$ would have reduced the peak hydrogen concentration to about 18,000 ppm, a $30 \%$ reduction.

After the tank is mixed, essentially all of the retained gas will have been released. Further mixer pump runs will not release large gas volumes, and no further BDGREs will be induced. Hydrogen concentrations will remain within a few hundred ppm of the baseline, and monitoring is not necessary as long as the mixer pump runs often enough to prevent gas retention. As 


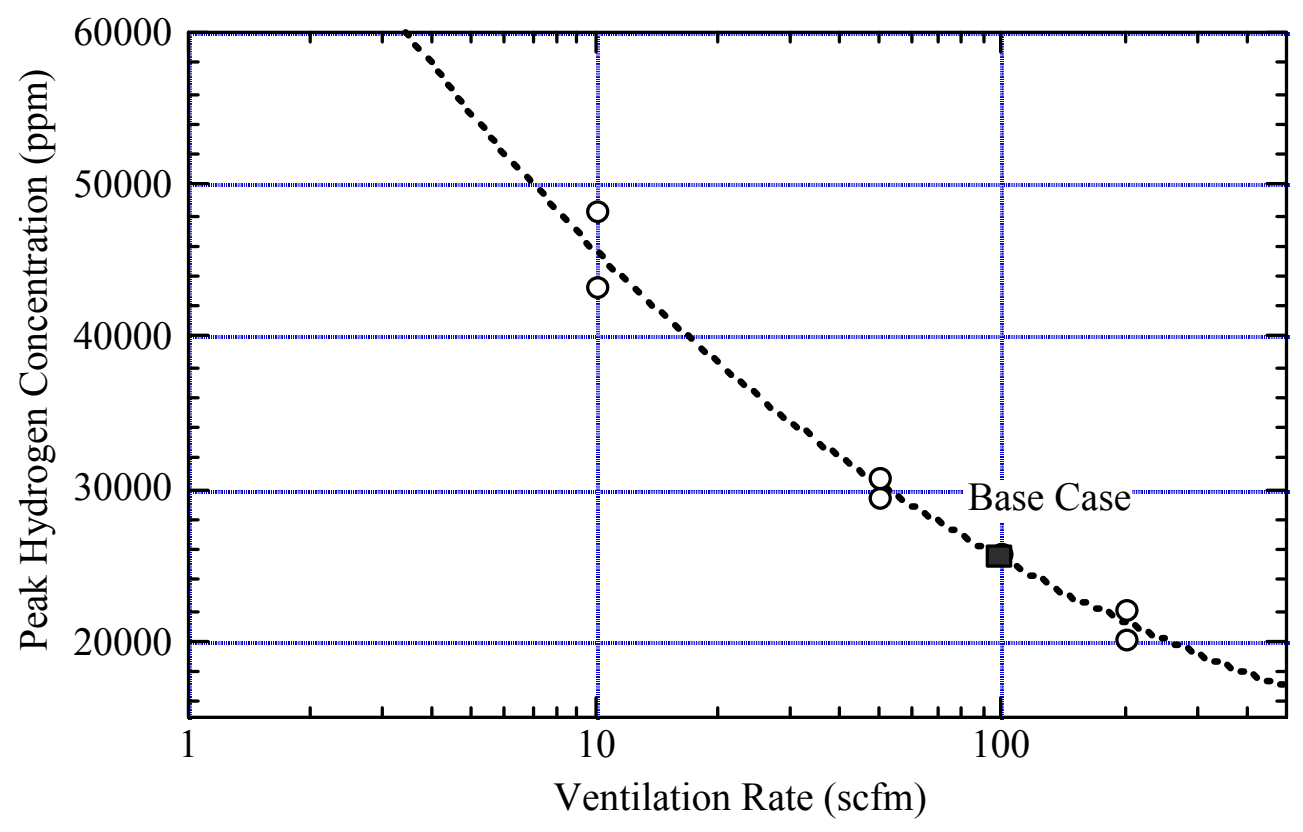

Figure 3.12. Sensitivity of Hydrogen Concentration to Ventilation in AN-103 During Mixing

mentioned in Section 3.6.2, it would take at least two years for a tank to accumulate enough gas to be classified in Waste Group B, which would require a degassing plan for mixing.

Gas monitoring is necessary in these tanks and in any DST in Waste Group A or B. No formal basis exists to calculate how long the monitoring period after pump operation should be, but 24 hours is a reasonable time based on the experience with SY-101 mixing and the mixing analysis presented in Section 3.6.2 (Allemann et al. 1994; Wells et al. 2002).

\subsubsection{Potential Changes in Tank Classification After Mixing}

In the long term, assuming that mixing is eventually terminated and the tank allowed to return to a steady state, no changes in waste configuration result from mixer pump operation, and tank classification will not change. ${ }^{\text {(a) }}$ However, if mixer pump operation continues frequently enough to keep gas from accumulating, a tank can be maintained indefinitely in Waste Group C, as was done with SY-101 for many years. Because mixing cannot move a tank into a higher waste group, classification need not be reevaluated due to mixer pump operation. However, if a tank is reclassified as Waste Group $\mathrm{C}$ on the basis that mixing prevents gas accumulation, continued mixer pump operation is required to keep the tank in Group C. See Table 3.6 for the effects of terminating mixing in Group A tanks.

(a) The effect of "fluffing," where the sediment layer is expanded while the previously mixed sediment gradually compacts to its former density, is discounted as a transient phenomenon compared with the minimum of four years before gas could accumulate to buoyancy (see Section 3.6.2). 


\subsubsection{Potential Changes in Hydrogen Generation After Mixing}

Because no waste is added or removed by mixing, the total hydrogen generation does not change, and no reanalysis of steady-state flammability is needed. However, because hydrogen generation is sensitive to the waste temperature, the effect of mixer pump power deposition in the waste should be considered for long-term operation.

\subsection{Airlift Circulator Operation}

Airlift circulators (ALCs) were designed into the construction of DSTs AY-101, AY-102, AZ-101, AZ-102, AN-107, and AW-102 as well as SSTs in several tank farms. ALCs mix the waste by introducing a stream of air bubbles into 30-inch-diameter cylindrical tubes that extend from near the tank bottom well up into the supernatant. The bubble stream reduces the average density inside the circulator tubes, causing an upward flow.

In the AY and AZ aging waste tank farms, each tank has 22 ALCs installed to prevent temperature excursions caused by settling of heat-generating fission products. AN-107 has 21 ALCs of the same design as those in the aging waste tanks designed to "gently agitate the waste." However, they were never connected to an air supply, and there are no plans to operate them in the future. AW-102 has only two ALCs that are much smaller (16 and 24 inch diameter) than in the other tanks that were intended to provide uniform feed for the 242-A evaporator.

The global disturbance induced by ALC operation is similar to that of mixing except that the intense hydraulic action of a high-velocity jet is absent. However, the simultaneous operation of many large-diameter ALCs, albeit at a relatively low velocity, tends to make up in volume for their lack of intensity.

\subsubsection{Mechanisms for Gas Releases During ALC Operation}

The highest gas release potential occurs during startup of ALCs. Establishing flow into ALCs with their bases buried in the waste requires mobilizing a large volume of gas-bearing sediment. This could release a large fraction of the retained gas volume. However, as with mixer pump operation, after the initial degassing period, ALC operation will not release gas unless the ALCs have been idle for a long period, allowing gas to re-accumulate.

As with mixing, the ALCs are designed to suspend sediment in the liquid, which reduces the neutral buoyancy gas fraction and could induce BDGREs. However, none of the Waste Group A tanks have operable ALCs, and the four tanks with operable ALCs are in Waste Group C, so induced BDGREs are not a concern. ${ }^{\text {(a) }}$

Stirring or agitating a liquid waste surface with a high concentration of dissolved ammonia greatly increases the evaporative ammonia mass transfer rate. The action of gas bubbles rising

(a) As described in Section 2.4.1, Waste Group A and B tanks store enough gas to make the headspace flammable if all of it were suddenly released; Waste Group C tanks do not. Waste Group A tanks exhibit spontaneous BDGREs, while tanks in Waste Group B do not. 
continuously in the ALCs is an ideal process for stripping ammonia from the liquid into the bubbles. The large ammonia releases during transfers of SY-101 waste into SY-102, which created the highest ammonia concentrations recorded by gas monitoring equipment, apparently involved gas bubble sparging of the waste stream in a vacuum break (Mahoney et al. 2000). This indicates that high headspace ammonia concentrations can be expected during ALC operation in waste with a high concentration of dissolved ammonia. The tanks with operable ALCs in the AY and AZ farms do not store high-ammonia waste at this time.

Figure 3.13 is a schematic of a typical ALC in the AY and AZ farms. The left panel of the figure depicts ALC operation within a sediment layer, and the right panel gives details of the design. The ALC diameter, $\mathrm{D}_{\mathrm{o}}$, is 30 in., and the base sits at height $\mathrm{H}_{\mathrm{o}}, 30$ inches above the tank bottom. The length, L, of 15 ALCs is $22 \mathrm{ft}$, but five are $17 \mathrm{ft}$ long. The air line has a diameter, $\mathrm{D}_{\mathrm{i}}$, of 6 in. that tapers down to a 1-inch pipe, terminating in a sparger that consists of four triangular notches cut into the end of the pipe. Each of these triangular openings has a hydraulic diameter, $d_{e}$, of approximately 0.33 in. Airflow can be adjusted from 0 to $20 \mathrm{scfm}$ in each ALC. Figure 3.14 shows the arrangement of ALCs in the AY and AZ tanks. One ALC is placed at the center, seven on a radius of $14.5 \mathrm{ft}$, and 14 at a $27-\mathrm{ft}$ radius, for a total of 22 in each tank.
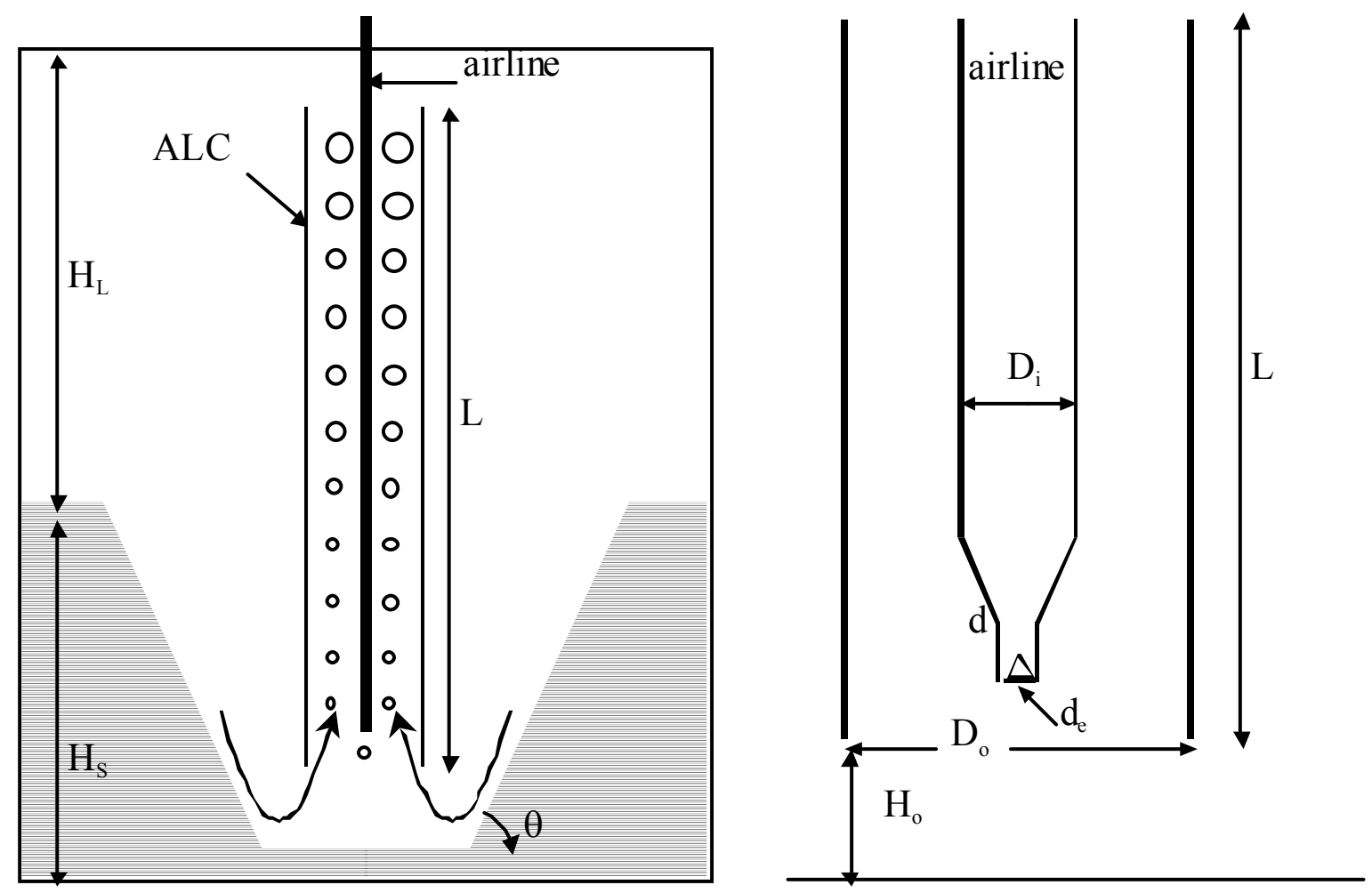

Figure 3.13. Schematic of Typical Airlift Circulator Operation 


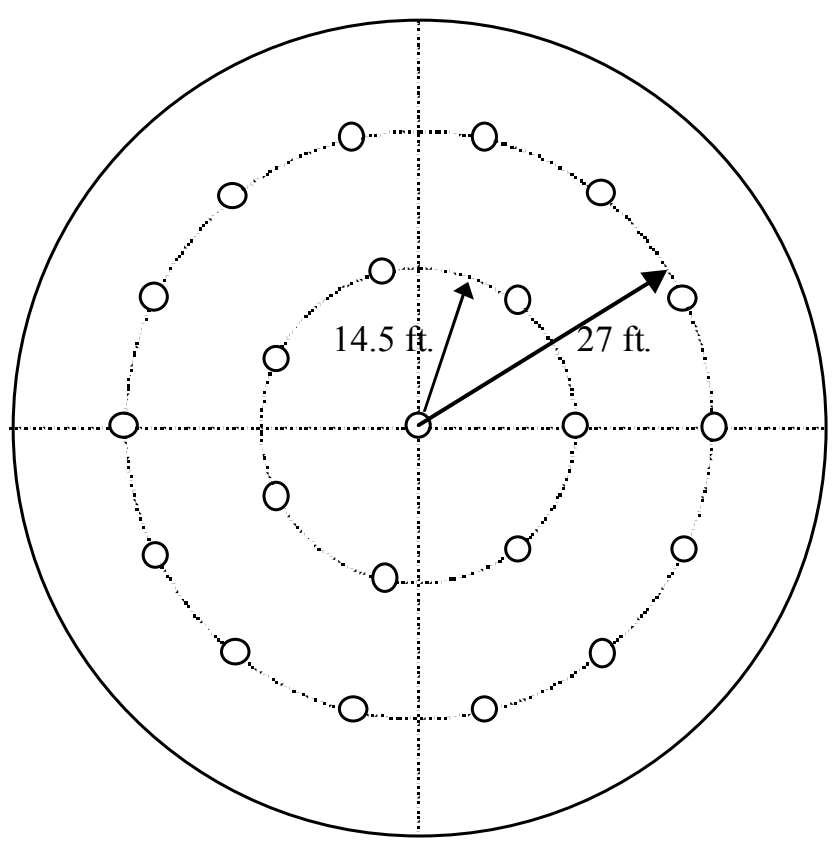

Figure 3.14. Location of ALCs in AY and AZ DSTs

To estimate the potential for gas releases induced by ALC operation, simple models were developed for airlift pump theory and mobilization of sediments. These models are applied to conditions representing Tanks AN-107 (saltcake waste) and AY-102, AZ-101, and AZ-102 (sludge wastes). AN-107 is included to represent the potential gas release if ALCs were run, even though they are not connected to an air supply and are not planned to be operated. AY-102 is included even though its waste depth is more than $1 \mathrm{~m}$ below the top of the 22-ft ALCs, which would prevent them from operating. AW-102 and AY-101 are excluded because they contain little waste. AW-102 has only two ALCs, and their operation is more of a local waste disturbance in any case. AZ-101, with its relatively shallow sediment layer, is included because its ALCs were run late 1999 and mid-2000.

\subsubsection{Expected Gas Releases During ALC Operation}

A sediment layer cannot be mixed and retained gas will not be released unless flow is established in the ALC. However, a sediment layer around the base of the ALC prevents flow unless the lithostatic pressure can be overcome and the material can be yielded by the pressure difference across the ALC tube. Anecdotal evidence is that the majority of the air lines are plugged in both AY and AZ farms, and water pressure in excess of 150 psi could not remove the plug. Even if the air lines are clear, the sediment may be too strong to allow circulation. Gas bubbles may escape around the outside of the ALC, or bubbles rising through the sediment inside the ALC may fail to mobilize the sediment therein. Seventeen ALCs were run in the recent operations in AZ-101. 
Assuming that the sediment strength is a few hundred $\mathrm{Pa}$, the startup pressure difference produced by $20 \mathrm{cfm}$ of airflow should be more than adequate to yield the sediment and initiate flow. Also, bubbles generated at the sparger should preferentially rise through any sediment inside the ALC rather than bypassing around the outside.

If ALC pumping can be initiated, the resulting flow should mobilize the sediment in the region of the ALC and release the stored gas. The likely evolution of waste disturbance due to a single ALC in operation is illustrated in Figure 3.15. When the ALC first starts up, it is likely that supernatant will be drawn from around its base and sides (frame 1 in Figure 3.15). Agitation from the initial startup process would likely create a loose slurry inside the ALC. Additionally, air bubbles that escaped and rose to the surface along the outside of the ALC would create paths to the supernatant layer for liquid flow. This situation would be unstable, however, and the weak sediment would begin to collapse around the outside. During this process (frame 2), entrained sediment would be pumped upward and discharge at the outlet, releasing gas in the process.

Sediment would continue to slough off and be entrained toward the inlet, and a stable configuration could eventually be attained (frame 3 ). A maximum angle of repose, $\theta$, exists for a given sediment strength that is stable. The maximum angle of repose is discussed further below. If the fluid velocities are high enough, fluid shear will continue to erode the sediment and the mobilized volume will increase. Finally (frame 4), the volume will become large enough and flow velocities reduced enough that fluid shear cannot erode any more material. This represents the maximum volume that could be disturbed by a single ALC. The size of the disturbed volume will depend on the sediment properties (strength, repose angle, critical shear stress for erosion, etc.) and the fluid velocities.

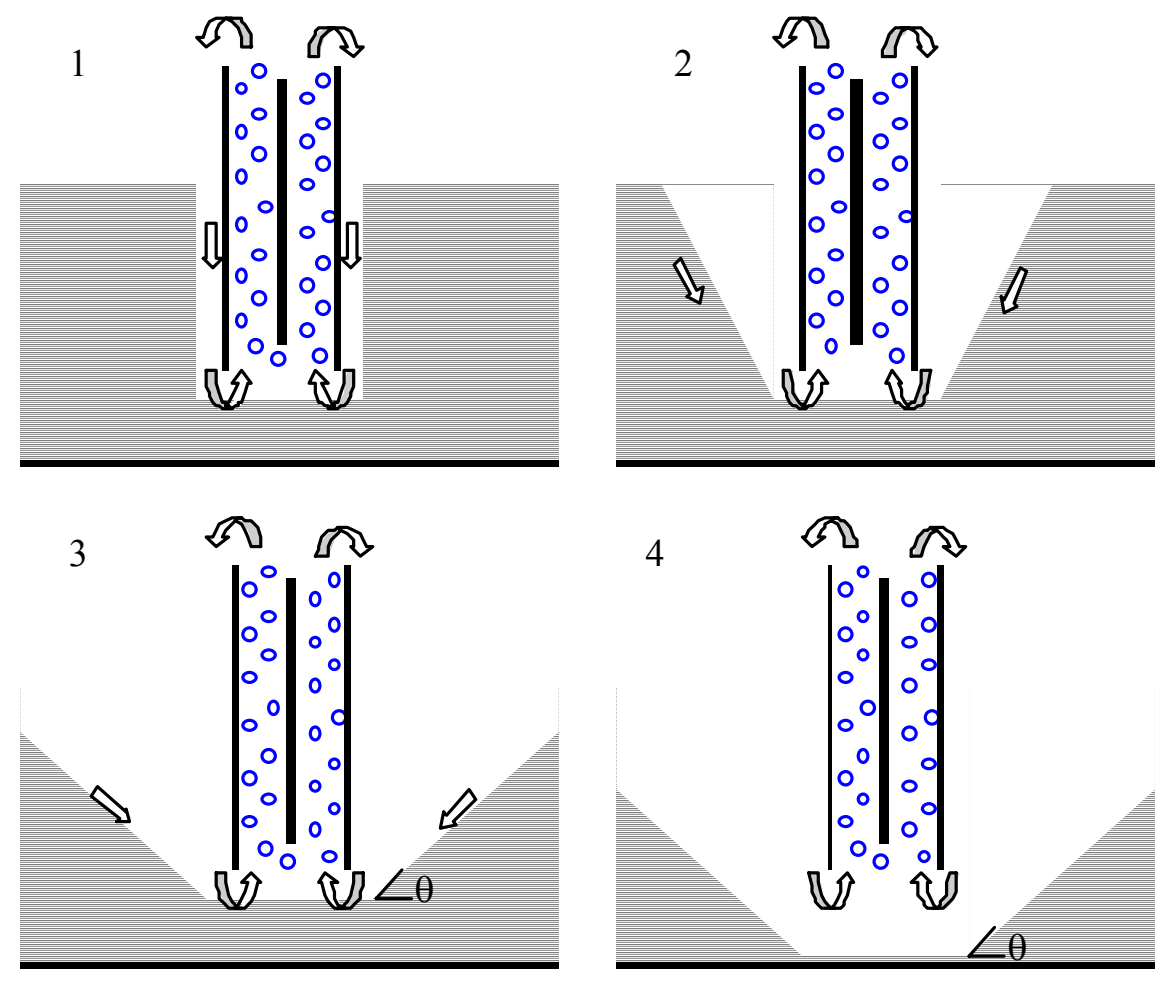

Figure 3.15. Stages of Waste Disturbance During ALC Operation 
An expression for the maximum angle of repose, $\theta$, for a weak granular sediment has been derived by Terrones ${ }^{(a)}$ and is given here:

$$
\tan \theta=\frac{8 \tau \Delta \rho g H}{(\Delta \rho g H)^{2}-16 \tau^{2}}
$$

where

$$
\tau=\text { sediment shear strength. }
$$

$\mathrm{H}=$ distance from the base of the erosion to the top of the sediment layer. If the sediment is eroded all the way to the tank floor, then $\mathrm{H}=\mathrm{H}_{\mathrm{S}}$.

$\Delta \rho=$ density difference between the sediment and the liquid above it.

During mixing, the supernatant density increases due to suspended solids. As this happens, the angle of repose increases, decreasing the rate of solids suspension. Thus, because suspension and resettling changes the preferred angle, which in turn changes the suspension rate, the system may oscillate and never reach a steady state. On the other hand, it is also possible that the system is highly damped and could quickly reach a steady state. Only further analysis and testing can determine which case applies.

The suction velocities and sediment properties determine the cleared base of the eroded conical volume around the ALC. To estimate the potential magnitude of the disturbed volume, assume the cleared base is equal to the diameter of the ALC. Hence, the eroded volume is a function of an inverted right circular cone with height $\mathrm{H}_{\mathrm{SL}}$, minor base radius $\mathrm{D}_{0} / 2$, and major base diameter given by

$$
\mathrm{D}=\mathrm{D}_{0}+2 \mathrm{H}_{\mathrm{S}} \tan (\pi / 2-\theta)
$$

The volume is

$$
\mathrm{V}=\frac{\pi}{12} \mathrm{H}_{\mathrm{S}}\left(\mathrm{D}^{2}+\mathrm{D}_{0}^{2}+\mathrm{DD}_{0}\right)
$$

The pertinent waste properties for the tanks analyzed are shown in Table 3.7. Assuming $\mathrm{H}=\mathrm{H}_{\mathrm{S}}$ and $\Delta \rho=\rho_{\mathrm{S}}-\rho_{\mathrm{L}}$, values of $\theta$ and the diameter and volume of the region disturbed by one ALC are shown in Table 3.8 for the four DSTs with ALCs selected for analysis. Also shown is the predicted gas release from a single ALC and the total for all 21 or 22 ALCs (17 in AZ-101) at standard pressure, computed from the disturbed volumes, gas volume fractions, and pressure ratios. Values for the radius and disturbed volume vary widely because (per Eq. 3.7.1) shallower sediment layers such as those in AZ-101 and AZ-102 have a high angle of repose, whereas deep sediments have a shallower angle, hence a larger volume. The small angles of repose in AN-107 and AY-102 result in the potential for almost the entire sediment volume to be disturbed by ALC operation. This releases much of the stored gas. The model hydrogen concentration results for

(a) Meyer PA, CW Stewart, SD Rassat, RT Allemann, G Terrones, and DP Mendoza. May 1999. Potential Gas Release by Bubble Slurry Flow Through a Hole in the Crust Layer in SY-101. Letter Report TWS99.27 Rev. 1, Pacific Northwest National Laboratory, Richland, WA. 
Table 3.7. Waste Properties in Tanks with ALCs (Barker and Hedengren 2002)

\begin{tabular}{||l|c|c|c|c||}
\hline Property & AN-107 & AY-102 & AZ-101 & AZ-102 \\
\hline Supernatant Depth $\mathrm{H}_{\mathrm{L}}(\mathrm{m})$ & 7.32 & 4.73 & 8.49 & 8.23 \\
\hline Sediment Depth $\mathrm{H}_{\mathrm{S}}(\mathrm{m})$ & 2.3 & 1.57 & 0.48 & 0.97 \\
\hline Supernatant Density $\rho_{\mathrm{L}}\left(\mathrm{kg} / \mathrm{m}^{3}\right)$ & 1370 & 1150 & 1240 & 1140 \\
\hline Bulk Sediment Density $\rho_{\mathrm{S}}\left(\mathrm{kg} / \mathrm{m}^{3}\right)$ & 1560 & 1397 & 1620 & 1380 \\
\hline Sediment Shear Strength $\tau{ }^{(\mathrm{a})}(\mathrm{Pa})$ & 200 & 200 & 200 & 200 \\
\hline Sediment Gas Volume Fraction $\alpha$ & 0.008 & 0.06 & 0.06 & 0.06 \\
\hline Gas pressure p $($ atm $)$ & 2.1 & 1.6 & 2.1 & 2.0 \\
\hline $\begin{array}{l}\text { (a) Shear strength is assumed similar to in situ values measured in Waste Group A tanks. In situ } \\
\text { shear strength values are considered more accurate than laboratory measurements. Further, the lower } \\
\text { in situ values are more conservative in waste mobilization with regard to gas release by ALCs. }\end{array}$ \\
\hline
\end{tabular}

Table 3.8. Disturbed Sediment Volumes

\begin{tabular}{||l|c|c|c|c||}
\hline \multicolumn{1}{|c|}{ Property } & AN-107 & AY-102 & AZ-101 & AZ-102 \\
\hline Angle of repose (degrees) & 21 & 24 & 48 & 39 \\
\hline Disturbed region diameter $(\mathrm{m})$ & 12 & 8 & 2 & 3 \\
\hline Disturbed volume - 1 ALC $\left(\mathrm{m}^{3}\right)$ & 98 & 29 & 1 & 3 \\
\hline Disturbed volume - all ALC $\left(\mathrm{m}^{3}\right)$ & 935 & 630 & 9 & 73 \\
\hline Total gas released - 1 ALC $\left(\mathrm{m}^{3}\right)$ & 2 & 3 & $<1$ & $<1$ \\
\hline Total gas released - all ALC $\left(\mathrm{m}^{3}\right)$ & 16 & 62 & 1 & 1 \\
\hline$\left[\mathrm{H}_{2}\right]$ - 1 ALC $(\mathrm{ppm})$ & 600 & 510 & 20 & 20 \\
\hline$\left[\mathrm{H}_{2}\right]$ - all ALC $(\mathrm{ppm})$ & 5,700 & 11,300 & 300 & 300 \\
\hline \hline
\end{tabular}

AZ-101 compare favorably with the recorded values for ALC operation in AZ-101 in May 2000 (430 ppm maximum hydrogen concentration) (Carlson et al. 2000). AY-102 is predicted to exceed $25 \%$ of the LFL. This potential for gas release indicates that ALC operation in Waste Group A or B tanks should be treated with the same care as mixer pump operation. However, ALC operations do not present a flammability issue (100\% LFL) where they may be operable (AY and AZ tanks) because those tanks are in Waste Group C.

The actual cleared area at the base of the ALCs will be strongly affected by the magnitude of the induced flow velocities in the region. Models for predicting the flow rate in the ALC exist in the literature. One model presented in (Hetsroni 1982) gives the nondimensional flow rate as

$$
\frac{\mathrm{Q}_{\mathrm{m}}^{2}}{2 \mathrm{~A}^{2} \mathrm{~g}\left(\mathrm{~L}-\mathrm{L}_{\mathrm{s}}\right)}=\frac{\alpha_{\mathrm{m}}-\left(1-\rho_{\mathrm{L}} / \rho_{\mathrm{S}}\right)+\left(1+\mathrm{L}_{\mathrm{e}} / \mathrm{L}_{\mathrm{s}}\right)}{\left(4 \mathrm{f} / \mathrm{D}_{\mathrm{h}}\right)\left[\mathrm{L}_{\mathrm{e}}+\mathrm{L}_{\mathrm{s}}\left(1-\alpha_{\mathrm{m}}\right)^{-1.75}\right]+2\left[1 /\left(1-\alpha_{\mathrm{m}}\right)-0.75\right]}
$$

where $\mathrm{Q}_{\mathrm{m}}$ is the flow rate of the slurry mixture in the ALC, A is the ALC cross-sectional area, $\alpha_{\mathrm{m}}$ is the average gas volume fraction in the rising column, $\rho_{\mathrm{m}}$ is the average density of the mixture, $\mathrm{D}_{\mathrm{h}}$ is the hydraulic diameter, and $\mathrm{f}$ is the friction factor. The length, $\mathrm{L}_{\mathrm{e}}$, is the distance between 
the inlet and the air discharge nozzle, and the term $\mathrm{L}_{\mathrm{s}}$ is the remaining length $\mathrm{L}_{\mathrm{s}}=\mathrm{L}-\mathrm{L}_{\mathrm{e}}$. The mixture gas fraction is depends on the flow rate and is given by

$$
\alpha_{m}=\frac{Q_{g}}{Q_{g}+Q_{m}+s A}
$$

where $\mathrm{Q}_{\mathrm{g}}$ is the gas flow rate and $\mathrm{s}$ is the relative slip between the rising air bubbles and the fluid. The evaluation of Eq. (3.7.4) and (3.7.5) is iterative, with the mixture gas volume fraction being adjusted until the equation balances.

Eq. (3.7.4) was evaluated for Tank AZ-102 conditions using a slip factor of 0.3 and a friction factor of 0.005 . Flow rate and average rise velocity $\left(Q_{m} / A\right)$ are plotted versus mixture density in Figure 3.16. The maximum flow rate occurs when no solids are entrained, so while it is not possible to quantify the disturbance size due to ALC operation exactly, we can make some useful observations regarding potential gas release rate. Because the flow rate is related to mixture density and mixture density is related to solids loading, we can relate ALC flow rate to gas release rate. The mixture is made up of convective liquid and sediment. Hence we can write

$$
\mathrm{Q}_{\mathrm{m}}=\mathrm{Q}_{\mathrm{L}}+\mathrm{Q}_{\mathrm{S}}
$$

where $\mathrm{Q}_{\mathrm{L}}$ is the supernatant flow rate and $\mathrm{Q}_{\mathrm{S}}$ is the flow rate of sediment. Mass conservation is expressed as

$$
\rho_{\mathrm{m}} \mathrm{Q}_{\mathrm{m}}=\rho_{\mathrm{L}} \mathrm{Q}_{\mathrm{L}}+\rho_{\mathrm{S}} \mathrm{Q}_{\mathrm{S}}
$$

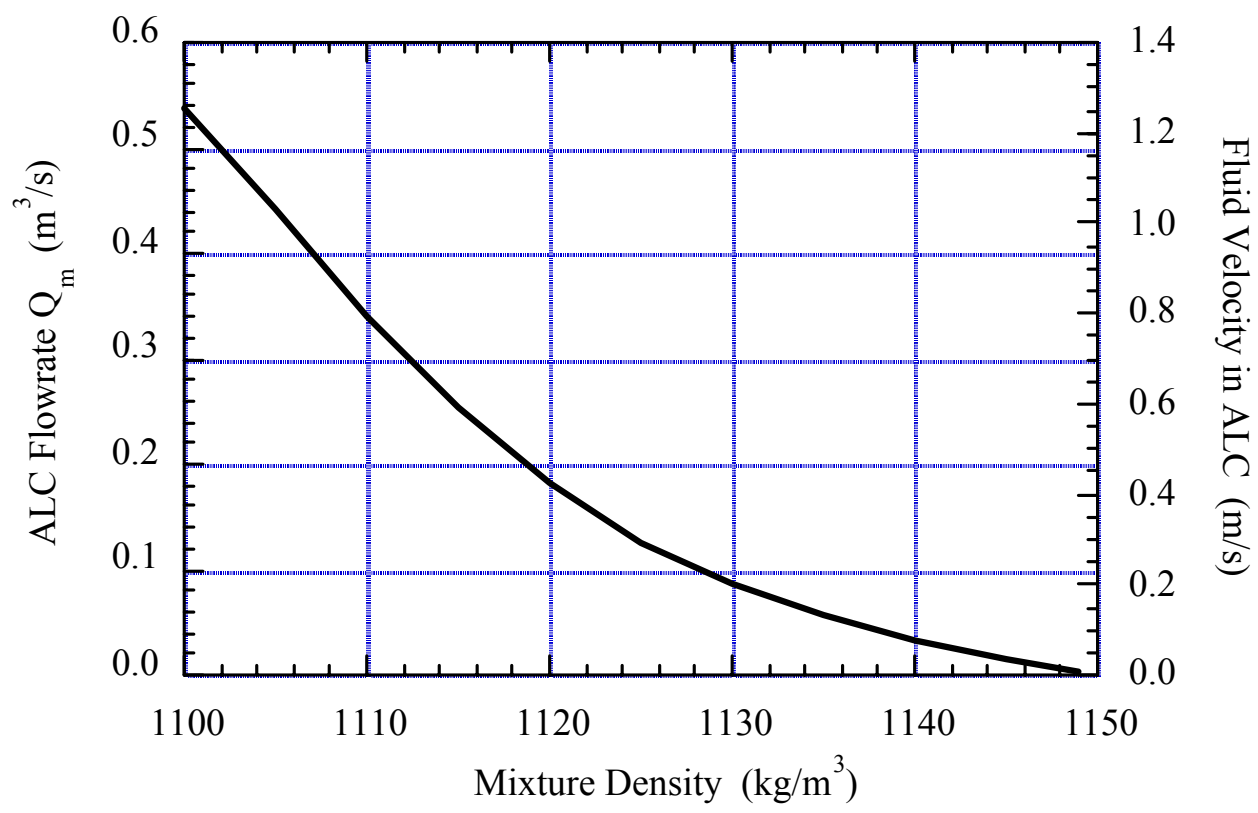

Figure 3.16. ALC Flow Rate and Velocity for Tank AZ-102 
Combining Eq. (3.7.6) and (3.7.7) we obtain the entrainment rate of sediment:

$$
\mathrm{Q}_{\mathrm{S}}=\frac{\rho_{\mathrm{m}}-\rho_{\mathrm{L}}}{\rho_{\mathrm{S}}-\rho_{\mathrm{L}}} \mathrm{Q}_{\mathrm{m}}
$$

The corresponding total gas release rate at standard conditions, $\mathrm{Q}_{\mathrm{REL}}$, is given by

$$
\mathrm{Q}_{\mathrm{REL}}=\gamma \frac{\rho_{\mathrm{S}}}{\rho_{\mathrm{A}}} \mathrm{Q}_{\mathrm{S}}
$$

The predicted gas release rate for one ALC operating in Tank AZ-102 is shown in Figure 3.17. The gas release rises with increasing mixture density until a maximum of about $2 \mathrm{cfm}$ is reached. The gas release then falls rapidly to zero as the ALC begins to stall.

The results of this analysis suggest that ALCs may or may not be able to start due to the strength of the sediment and the modest lift pressures that can be achieved by $20 \mathrm{scfm}$ of air flow. If started, fairly large waste disturbances are possible because the weak sediment can slough toward the inlet of the ALCs. However, analysis suggests that the flow velocities in the ALCs are relatively small, and even small solids loadings will tend to stall the ALCs. This result allows us to conclude that the gas release rate must be relatively low because the ALCs can only accommodate very low solids loadings.

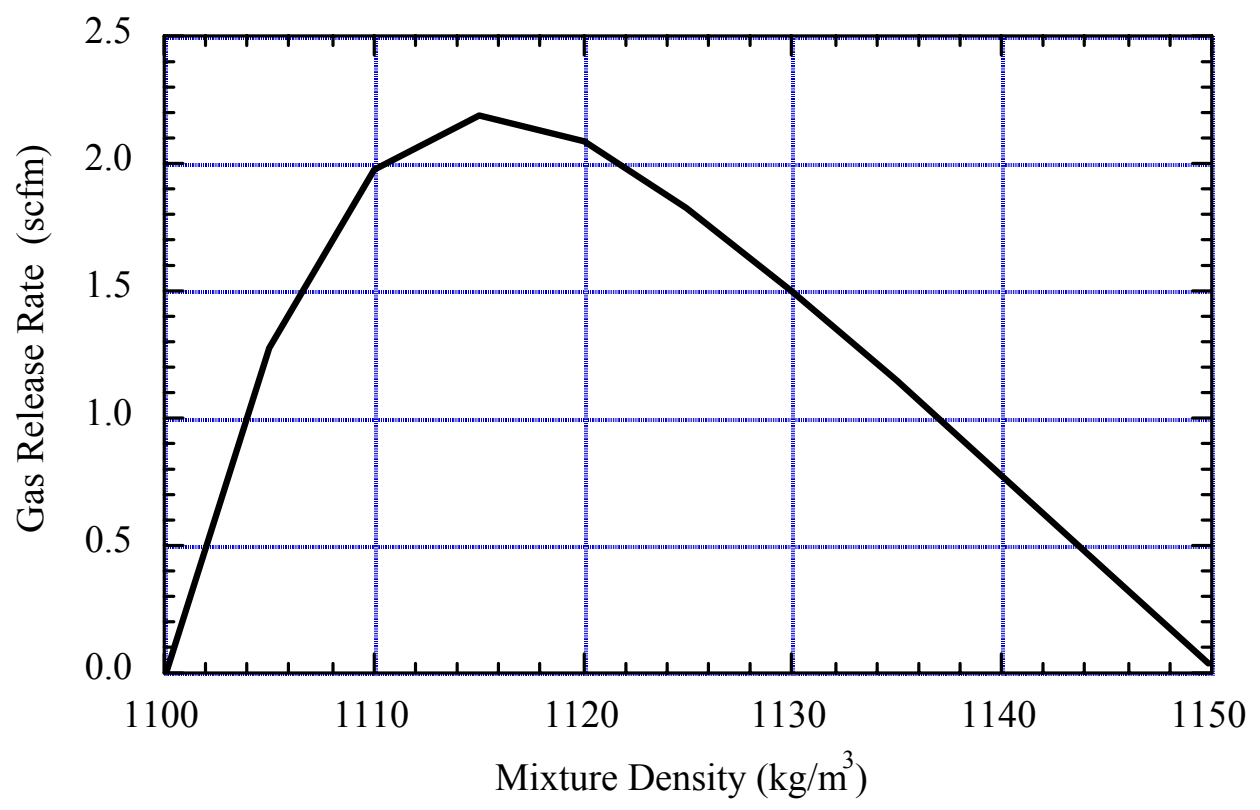

Figure 3.17. Gas Release Rate from a Single ALC in Tank AZ-102 


\subsubsection{Gas Monitoring Considerations During ALC Operation}

Based on the analysis above, the gas release rate during ALC operation is likely to be limited. However, if one of the tanks with operable ALCs were placed in Waste Group A or B at some time in the future, the total gas release could bring the headspace to the LFL. Besides the gas released directly by the waste disturbance, it is also possible to induce BDGREs as the suspended sediment reduces the neutral buoyancy gas fraction. These events would add a rapid release rate component to that caused by the ALCs that could quickly drive the headspace hydrogen concentration above the action level. Ammonia would also be released at a potentially high rate depending on the airflow, whether the ALC started or not (see Section 3.7.1). This suggests that gas monitoring is necessary for ALC operation in Waste Group A and B tanks, that the monitoring should be continuous, and that some kind of degassing plan should be applied. However, all tanks with potentially operable ALCs are now in Waste Group C, where no gas monitoring is needed during ALC operation.

\subsubsection{Potential Changes in Tank Classification After ALC Operation}

Because, like mixing, ALC operation does not add or remove waste from the tank or change the waste configuration in the long term, no waste group change is possible. ${ }^{\text {(a) Hence reevalu- }}$ ation of waste group placement is not required. After initial degassing, periodic ALC operation would prevent gas accumulation and maintain the tank in Waste Group C if it were reclassified.

\subsubsection{Potential Changes in Hydrogen Generation After ALC Operation}

Hydrogen generation rates will not change as a result of ALC operation. Also, unlike mixer pump operation, the ALCs tend to be self-cooling and do not heat the waste appreciably. Reevaluation of hydrogen generation is not required.

\subsection{Chemical Addition}

Concentrated sodium hydroxide and possibly sodium nitrite solutions need to be added to DSTs occasionally to keep the waste chemistry within corrosion control limits. Additions are typically on the order of 50,000 gallons of solution with a specific gravity on the order of 1.5 (Fort 2001). No chemical additions are anticipated in SSTs except possibly during retrieval. Addition of chemicals other than for corrosion protection is not permitted at this time.

The global disturbance is a relatively small increase in the hydrostatic pressure, the same as a waste addition, along with an increase in the supernatant depth. However, the high density of the solution also increases the bulk density of the supernatant, thereby reducing the neutral buoyant gas fraction. Depending on waste chemistry, solids may be dissolved or precipitated.

(a) The effect of "fluffing," where the sediment layer expands and the previously mixed sediment gradually compacts to its former density, is discounted as a transient phenomenon compared to the minimum of four years before gas could accumulate to buoyancy (see Section 3.6.2). 


\subsubsection{Mechanisms for Gas Release During Chemical Addition}

Additions of caustic and sodium nitrite do not cause chemical reactions that release gas. The only mechanism for appreciable gas release is the decrease in neutral buoyancy void fraction resulting from mixing the relatively heavy caustic solution in the supernatant. This would likely induce a BDGRE in a Waste Group A tank ${ }^{(\mathrm{a})}$ and possibly in a borderline Group B tank for large-volume additions.

The analysis for chemical addition is exactly the same as that described for waste addition in Section 3.1. In Group A tanks, induced BDGREs can be avoided by ensuring that the density of the solution added is less than that of the existing supernatant. In Groups B and C tanks, induced BDGREs can be prevented by adjusting the addition to prevent spontaneous BDGREs in the future based on an evaluation of post-operation tank classification via Eq. (2.13). The likelihood of enhancing ammonia evaporation is less for chemical addition because added waste usually contains ammonia.

The temperature of chemical additions may be higher than that of the waste, which causes retained gas in the sediment to expand so that it could theoretically become buoyant. However, as discussed in Section 3.2, a very large temperature change would be needed to cause buoyancy, and temperature change is not considered a serious gas release mechanism.

\subsubsection{Expected Gas Releases During Chemical Addition}

No specific analysis has been performed on gas release rates and volumes of BDGREs induced in Group A tanks during chemical addition. As with waste addition (Section 3.2), the initial gob size of BDGREs induced during chemical addition should be similar to that of spontaneous releases. The peak hydrogen concentration would be increased above historical norms by the smaller headspace volume and higher gas release fractions. However, the increase would not be sufficient to make any of the current Waste Group A tanks exceed the LFL, as shown in Section 3.5.4.

There has been no experience or analyses on the size of BDGREs induced in Group B or C tanks that have not experienced them in the past. In the absence of historic BDGREs the waste should be more uniform, so the first induced BDGRE might be larger than indicated by the behavior of the current Group A tanks. If the waste addition is adjusted to prohibit BDGREs, no large gas release is expected from any other mechanism.

\subsubsection{Gas Monitoring Considerations During Chemical Addition}

No monitoring is required in Group B tanks as long as the addition is adjusted to prevent creating a new Waste Group A tank, which also prevents gas releases during the addition. Spontaneous BDGREs can occur at any time in Waste Group A tanks, and chemical addition

(a) As described in Section 2.4.1, Waste Group A and B tanks store sufficient gas to make the headspace flammable if all of it were suddenly released; Waste Group C tanks do not. Waste Group A tanks exhibit spontaneous BDGREs, while tanks in Waste Group B do not. 
would exacerbate the resulting hydrogen concentration somewhat, though not near the LFL. However, there is no effective control for spontaneous BDGREs, even if one were detected during the operation.

\subsubsection{Potential Changes in Waste Classification After Chemical Addition}

The initial calculations by Barker and Hedengren (2002) include potential changes in waste group classification from hypothetical additions of 10,000 gallons of water and caustic for all tanks. This small addition caused no changes in tank classification. However, caustic additions for corrosion control are likely to be much larger. Table 3.9 shows the change in buoyancy ratio resulting from a chemical addition of 100,000 gallons with a specific gravity of 1.5 in four DSTs representing Waste Groups B and C. The buoyancy ratios before and after the operation are calculated using Eq. (2.13). The tanks chosen represent the maximum buoyancy ratio values for their respective waste classification prior to caustic addition. This caustic addition causes the buoyancy ratio to exceed unity only in AW-105 and AY-102, but these tanks would remain in Waste Group $\mathrm{C}$ because of their large headspace. The buoyancy ratio increases slightly in AN107 and AW-103. Note that no change in sediment depth due to solid dissolution and/or precipitation is considered.

Table 3.9. Change in Buoyancy Ratio for Addition of $100 \mathrm{kgal}$ at $\mathrm{SpG}=1.5$

\begin{tabular}{||c|c|c|c||}
\hline \hline Tank & Group & \multicolumn{2}{|c||}{ Buoyancy Ratio } \\
\hline & & Before & After \\
\hline AN-107 & B & 0.93 & 0.95 \\
\hline AW-103 & B & 0.56 & 0.60 \\
\hline AW-105 & C & 0.48 & 1.3 \\
\hline AY-102 & C & 0.97 & 1.2 \\
\hline
\end{tabular}

This exercise shows that it is potentially possible to move a tank into or to the borderline of Waste Group A with caustic addition even without a potential increase in sediment depth due to solids precipitation. Therefore, an evaluation of the end-state tank classification is required for caustic additions to Group B and $\mathrm{C}$ tanks. Caustic additions to Group A tanks are not considered because the waste chemistry in all tanks exhibiting BDGREs is within corrosion control limits.

\subsubsection{Potential Changes in Hydrogen Generation After Chemical Addition}

A chemical addition to a tank adds to the total liquid inventory and adds to the amount of water available for radiolysis. It is not known whether increasing the hydroxide concentration would increase gas generation by itself or by dissolving solids, which would increase the aluminum and salt concentrations in the liquid. To accommodate the potential for changes, the hydrogen generation rate must be reevaluated for chemical additions. 


\subsection{Natural Evaporation}

Evaporation of water from the waste historically has not been considered a flammable gas issue but is treated here for completeness. It occurs naturally in all tanks but is important only in tanks with a relatively dilute supernatant and a high heat loading in the sediment. Significant evaporation has not been observed in concentrated saltcake tanks regardless of heat loading with either passive or active headspace ventilation. The presence of a floating crust layer further inhibits evaporation in the current Waste Group A tanks. ${ }^{\text {(a) }}$ In fact, psychometric data showed that the DST SY-101 actually absorbed water for long periods before it was remediated by transfer and dilution in early 2000. Evaporation is a potential issue only for DSTs whose waste configuration would make them susceptible to BDGREs. Reduction of the liquid content by evaporation is considered beneficial in SSTs with waste in the wet sediment or pumped configuration.

Evaporation causes two kinds of global disturbance. Slowly removing water from the supernatant and lowering the waste level decreases the hydrostatic pressure on the sediment and increases the density of the supernatant.

\subsubsection{Mechanisms for Gas Releases During Evaporation}

Evaporation of water from the waste does not itself release retained gas. However, it increases the concentration of dissolved solids in the supernatant, thereby raising its density. Carried to an extreme, the increasing supernatant density may reduce the neutral buoyancy gas fraction sufficiently to allow BDGREs. However, the reduction in supernatant depth would also tend to reduce the size of gas releases or prevent them altogether. At the same time, the increased salt concentration in the liquid reduces evaporation, so the process tends to be selflimiting.

If a BDGRE occurs due to a reduction in the neutral buoyancy gas fraction, it will suspend sediment in the supernatant, further reducing the neutral buoyancy gas fraction to potentially induce additional BDGREs. Decreasing hydrostatic pressure, which expands retained gas, would also act to promote gas release. However, the process of evaporation is so slow that the expanded bubbles would be released long before this could have an effect.

Though a reduction in neutral buoyancy void fraction resulting from evaporation would most likely induce a BDGRE in a Group A tank, the tanks that currently exhibit BDGREs have a very high salt concentration along with a floating crust layer, and their waste level history shows no evidence of significant evaporation (Hedengren et al. 2000). Evaporation might eventually induce a BDGRE in a borderline Group B tank; however, the tanks most likely to be borderline tend to have the most concentrated supernatant and therefore the least evaporation. The tanks with a high evaporation rate have a very dilute supernatant and are not likely to become an issue.

(a) As described in Section 2.4.1, Waste Group A and B tanks store sufficient gas to make the headspace flammable if all of it were suddenly released; Waste Group C tanks do not. Waste Group A tanks exhibit spontaneous BDGREs, while tanks in Waste Group B do not. 
In either case, the analysis performed in Section 3.2 for waste addition also applies to evaporation, which is simply the reverse of addition. The possibility of BDGREs induced by evaporation can be predicted and prevented if the effects of evaporation are evaluated and halted before the tank classification moves into Group A. The evaluation method is developed following the derivation given in Section 3.2.1. Figure 3.18 shows a sketch of the waste configuration before and after evaporation assuming no precipitation of solids. We assume a volume $V_{\mathrm{W}}$ of water with density $\rho_{\mathrm{W}}$ leaves the supernatant, which has an initial density $\rho_{\mathrm{L} 1}$ and thickness $\mathrm{H}_{\mathrm{L} 1}$. After evaporation, the concentrated supernatant will have decreased thickness $\mathrm{H}_{\mathrm{L} 2}$ and increased density $\rho_{\mathrm{L} 2}$. The thickness of the gas-retaining sediment layer will increase slightly from $\mathrm{H}_{\mathrm{S} 1}$ to $\mathrm{H}_{\mathrm{S} 2}$ due to the expansion of the retained gas from decreased hydrostatic pressure. The gas volume fraction will increase from $\alpha_{1}$ to $\alpha_{2}$ in the process.

The mixture density is expressed in terms of the evaporated water volume and initial quantities:

$$
\rho_{\mathrm{L} 2}=\frac{\rho_{\mathrm{L} 1} \mathrm{~V}_{\mathrm{L} 1}-\rho_{\mathrm{W}} \mathrm{V}_{\mathrm{W}}}{\mathrm{V}_{\mathrm{L} 1}-\mathrm{V}_{\mathrm{W}}}, \mathrm{V}_{\mathrm{W}}<\mathrm{V}_{\mathrm{L} 1}
$$
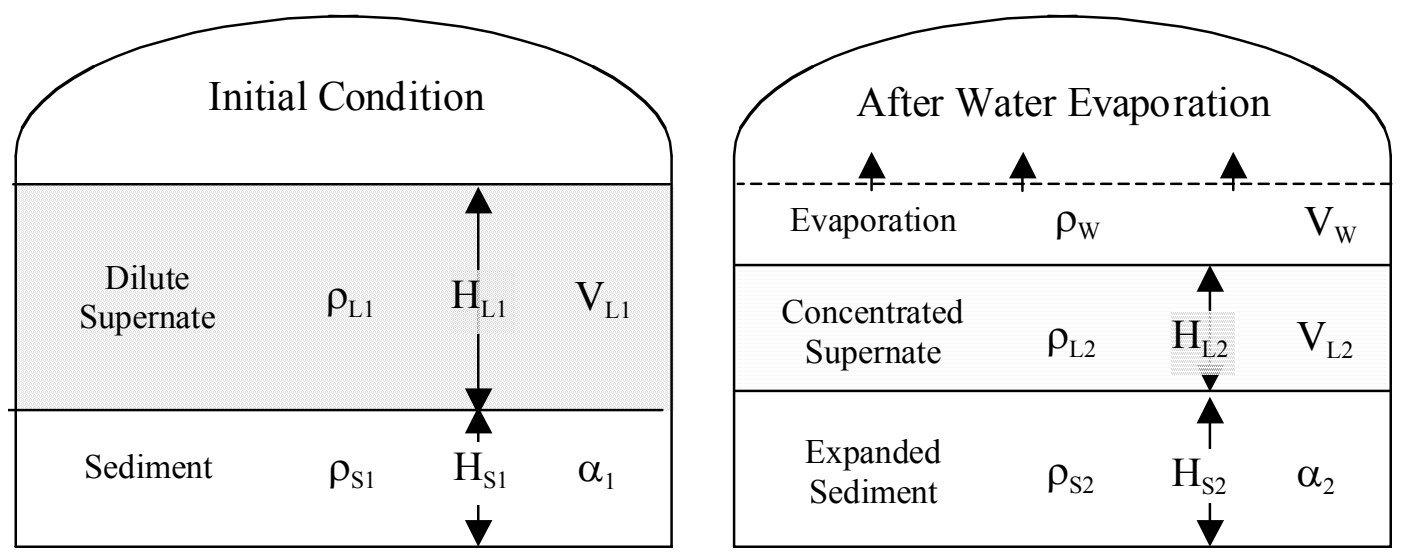

Figure 3.18. Tank Waste Configuration Before and After Evaporation

Combining Eq. (2.12) for the neutral buoyancy gas fraction with Eq. (3.1.2) for the final gas volume fraction provides an expression for the buoyancy ratio after evaporation:

$$
\mathrm{BR}_{2}=\frac{\alpha_{2}}{\alpha_{\mathrm{NB} 2}}=\frac{\alpha_{1} \rho_{\mathrm{S}} /\left(\rho_{\mathrm{S}}-\rho_{\mathrm{L} 2}\right)}{\alpha_{1}+\left(1-\alpha_{1}\right) \mathrm{P}_{2} / \mathrm{P}_{1}}
$$

The pressure ratio is given by Eq. (3.2.4) using the final density from Eq. (3.9.1) and the final supernatant depth computed by Eq. (3.2.2) with $\mathrm{V}_{\mathrm{T}}$ replaced by $\mathrm{V}_{\mathrm{W}}$. Substituting the product of the initial buoyancy ratio, Eq. (2.13), and the initial neutral buoyancy gas fraction, Eq. (2.12), for the initial gas fraction yields 


$$
\mathrm{BR}_{2}=\frac{\frac{\rho_{\mathrm{S}}}{\rho_{\mathrm{S}}-\rho_{\mathrm{L} 2}}}{1+\left[\frac{\rho_{\mathrm{S}}}{\mathrm{BR}_{1}\left(\rho_{\mathrm{S}}-\rho_{\mathrm{L} 1}\right)}-1\right] \frac{\mathrm{P}_{2}}{\mathrm{P}_{1}}}
$$

If the final buoyancy ratio is greater than unity, a BDGRE is likely.

For evaporation, using Eq. (3.9.1) and (3.9.3) yields a larger final buoyancy ratio than that calculated directly with Eq. (2.13), which is used to evaluate the buoyancy ratio for waste group selection. However, the derivation of Eq. (3.9.3) assumes that no gas escapes from the sediment during evaporation, so all gas present initially expands as the pressure decreases. This is not a reasonable assumption for the long period over which a large volume of waste would evaporate. Therefore, the reevaluation of the tank classification and adding liquid to reverse the effects before evaporation moves the tank into Group A will also be sufficient to prevent inducing BDGREs during evaporation.

\subsubsection{Expected Gas Releases During Evaporation}

Evaporation does not itself release retained gas. The only gas release hazard associated with evaporation is the potential for eventually creating a Group A tank. However, the process is very slow and can be reversed easily by adding back makeup water. Evaporation is negligible in the current Group A tanks and should not affect their current spontaneous BDGRE behavior.

There has been no experience or analyses on the potential size of BDGREs induced in Group $\mathrm{B}$ or $\mathrm{C}$ tanks that have not experienced them in the past. In the absence of historic BDGREs, the waste should be more uniform, so the first induced BDGRE might be larger than indicated by the behavior of the current Group A tanks.

\subsubsection{Gas Monitoring Considerations During Evaporation}

Evaporation is a chronic, long-term effect whose main adverse consequence is increasing the density of supernatant and a potentially changing tank classification. Because no gas release is associated with evaporation itself, no gas monitoring is required. The waste level is monitored in all tanks so the cumulative effects of evaporation can be tracked in tanks where it is of concern.

\subsubsection{Potential Changes in Tank Classification After Evaporation}

The amount of evaporation needed to cause a BDGRE was evaluated for four DSTs currently in Waste Groups B and C. Figure 3.19 shows the buoyancy ratio calculated with Eq. (3.9.2) versus the volume of water evaporated in Tanks AN-102 and AY-102 in Group C and AW-103 and AN-107 in Group B. The parameters used in the calculation are listed in Table 3.10. The waste level history indicates that the evaporation rate in all tanks but AY-102 is negligible. Evaporation is steadily reducing the waste level in AY-102 on the order of $30 \mathrm{~cm}$ ( 32,500 gallons) per year. 


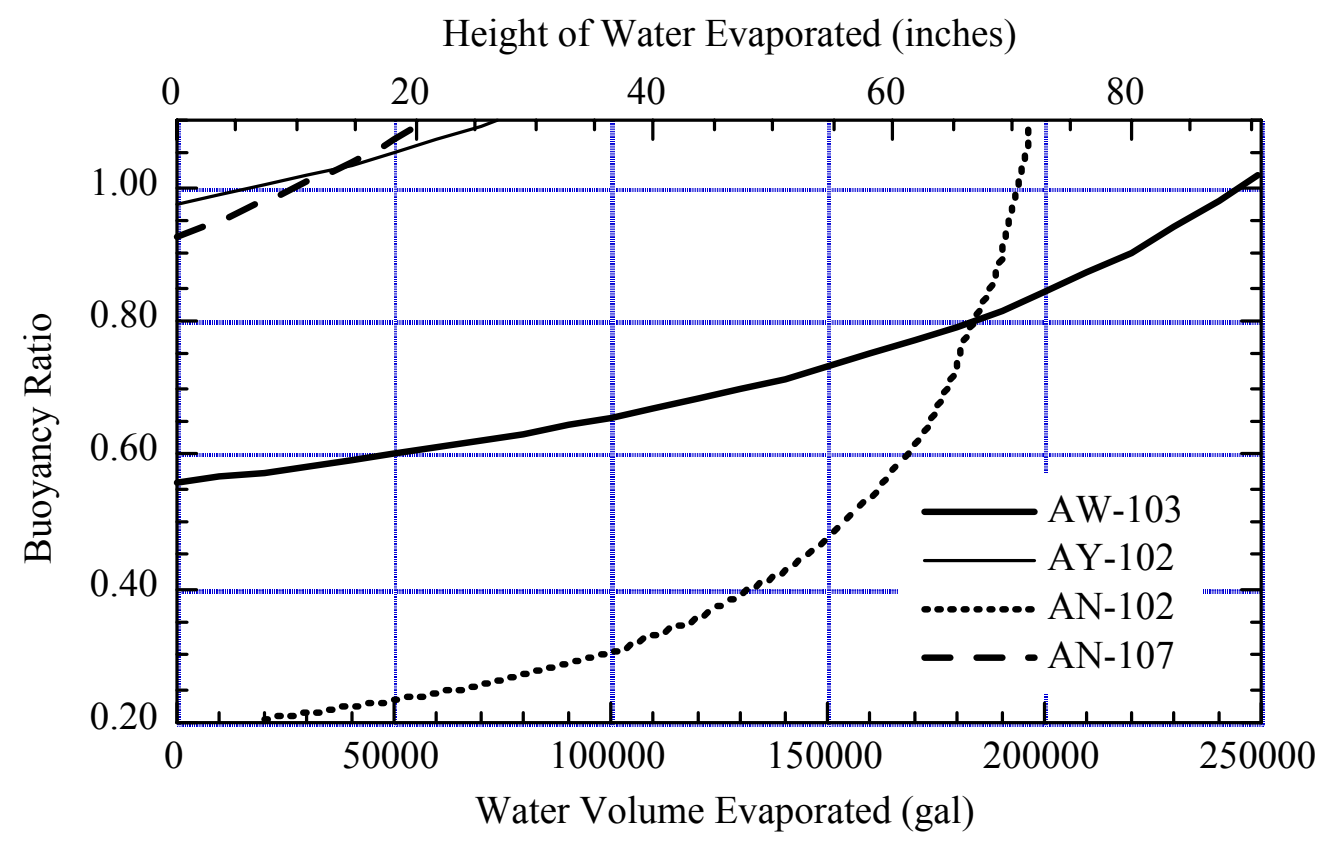

Figure 3.19. Effects of Evaporation on Buoyancy Ratio

Table 3.10. Tank Parameters Used for Evaporation Analysis (Barker and Hedengren 2002)

\begin{tabular}{||c|c|c|c|c||}
\hline Property & AN-102 & AW-103 & AY-102 & AN-107 \\
\hline $\mathrm{H}_{\mathrm{L} 1}(\mathrm{~m})$ & 9.1 & 7.3 & 4.7 & 7.3 \\
\hline $\mathrm{H}_{\mathrm{S}}(\mathrm{m})$ & 0.8 & 2.9 & 1.6 & 2.3 \\
\hline$\rho_{\mathrm{L} 1}\left(\mathrm{~kg} / \mathrm{m}^{3}\right)$ & 1430 & 1240 & 1150 & 1370 \\
\hline$\rho_{\mathrm{S}}\left(\mathrm{kg} / \mathrm{m}^{3}\right)$ & 1560 & 1498 & 1397 & 1560 \\
\hline$\alpha_{\mathrm{NB} 1}$ & 0.08 & 0.17 & 0.18 & 0.12 \\
\hline $\mathrm{BR}_{1}$ & 0.19 & 0.56 & 0.97 & 0.93 \\
\hline
\end{tabular}

Because the supernatant in $\mathrm{AN}-102$ and $\mathrm{AN}-107$ is already somewhat concentrated, evaporation rapidly increases the supernatant density, reducing the neutral buoyancy gas fraction and increasing the buoyancy ratio. Eq. (3.9.2) predicts that AN-107 would reach a buoyancy ratio of 1.0 after evaporation of 30,000 gallons or 10 inches of water. AN-102 could begin exhibiting BDGREs (though it would remain in Group C) after about 190,000 gallons or 72 inches of water have evaporated. AY-102 and AW-103 have a more dilute supernatant, so evaporation changes the buoyancy ratio more slowly. About 20,000 gallons or 7 inches of evaporation would need to evaporate to create a BDGRE in AY-102; AW-103 would require about 250,000 gallons, or 90 inches of evaporation.

This analysis shows that evaporation can, in theory, change a tank's classification, though a large amount of evaporation would be required. AY-102 is a possible exception. Because its initial buoyancy ratio is very close to unity and it has a relatively rapid evaporation rate, it would take less than a year to observe BDGREs in AY-102. However, this is not a concern because the tank is in Waste Group C. Though AN-107 requires less evaporation to make this change, about a year of evaporation at AY-102's high rate would be required to increase its buoyancy ratio to 
unity. Waste levels are monitored daily, so such trends would be obvious long before serious concentration occurred, and makeup water could be added to prevent it.

The self-limiting nature of evaporation can be evaluated by correlating the water partial pressure in a concentrated salt solution with the solution density. The water partial pressures measured by Norton and Pederson (1994) in simulated DST wastes were correlated with approximate densities derived from simulant compositions. The resulting correlation is

$$
\mathrm{P}_{\mathrm{H} 2 \mathrm{O}}=\left(\rho_{\mathrm{L}}-1\right)^{-0.00681} \exp \left[13.51-0.0038\left(\rho_{\mathrm{L}}-1\right) \mathrm{T}-1.669\left(\rho_{\mathrm{L}}-1\right)^{2}-5064 / \mathrm{T}\right]
$$

where

$\mathrm{P}_{\mathrm{H} 2 \mathrm{O}}=$ water partial pressure above the solution (atm)

$\rho_{\mathrm{L}} \quad=$ density of the salt solution $(\mathrm{g} / \mathrm{mL})$

$\mathrm{T} \quad=$ temperature of the solution $(\mathrm{K})$.

The water partial pressure in the waste given by Eq. (3.9.4) is plotted versus liquid density in Figure 3.20 for solution temperatures of 300,310 and $320 \mathrm{~K}$ (approximately $80^{\circ}, 100^{\circ}$ and $\left.120^{\circ} \mathrm{F}\right)$. There is approximately a factor of 3 decrease in water partial pressure going from a density of 1.0 to $1.5 \mathrm{~g} / \mathrm{mL}$. The maximum evaporation rate will occur in the area where ambient air is first exposed to the waste surface. A representative partial pressure for water vapor in the Hanford atmosphere ${ }^{(a)}$ is on the order of $0.03 \mathrm{~atm}$, which is shown in the figure. The maximum rate of evaporation is roughly proportional to the difference between the equilibrium partial pressure of the waste and the partial pressure of water in the incoming air. This indicates that the

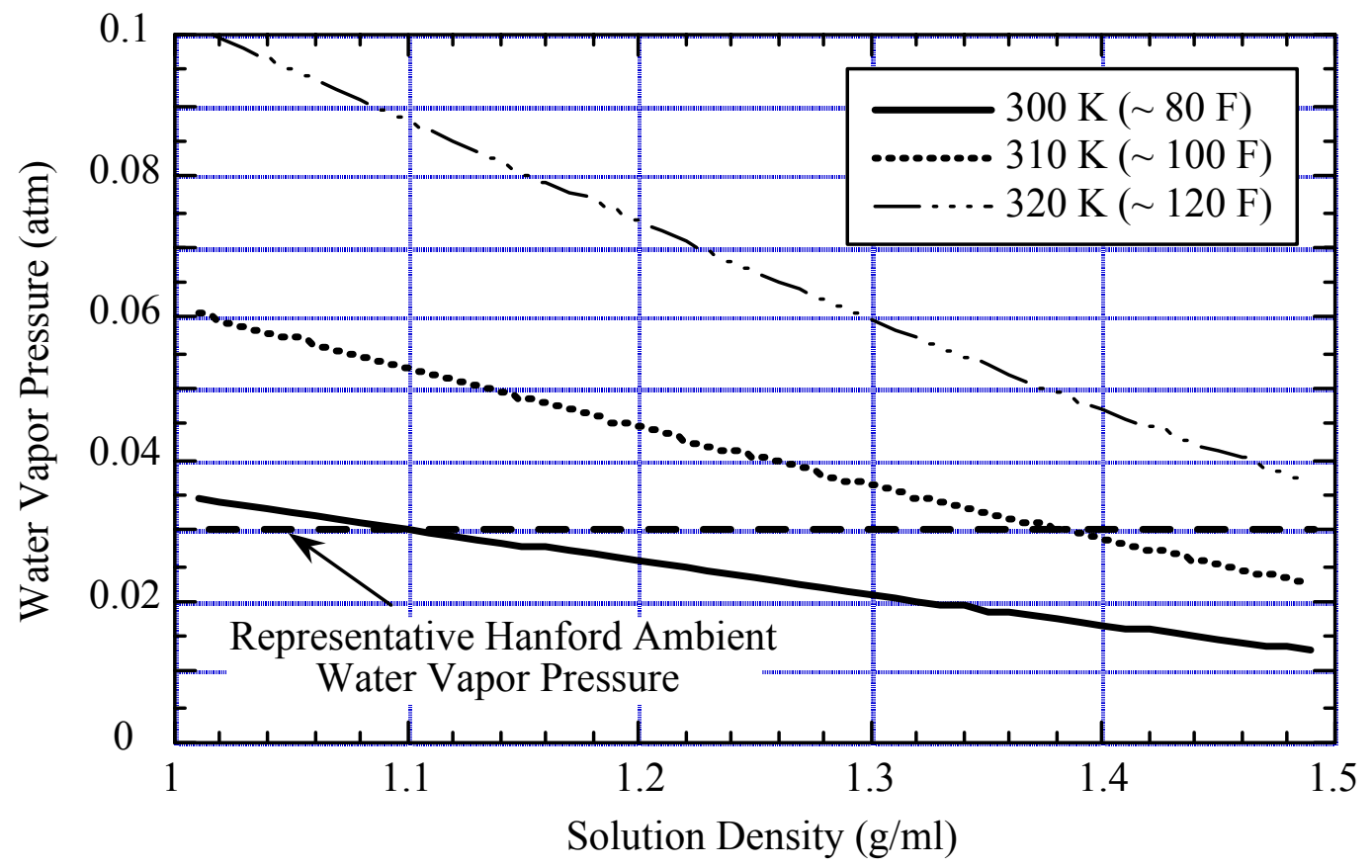

Figure 3.20. Water Partial Pressure Versus Solution Density

(a) Mahoney LA. April 2000. Modeling Evaporation from the 241-AX-152 Catch Tank. Letter report TWS00.44, Pacific Northwest National Laboratory, Richland, WA. 
evaporation rate will become small for solution densities above about $1.1 \mathrm{~g} / \mathrm{mL}$ at $300 \mathrm{~K}$ and roughly $1.4 \mathrm{~g} / \mathrm{mL}$ at $310 \mathrm{~K}$. Evaporation also decreases strongly at higher densities at $320 \mathrm{~K}$ even though it does not go to zero.

Because evaporation effectively ceases before it can raise the liquid density to the point where BDGREs would occur, we conclude that evaporation does not pose a significant risk.

\subsubsection{Potential Changes in Hydrogen Generation After Evaporation}

Because evaporation removes water from the tank that would have been subject to radiolysis, the gas generation rate should decrease. Therefore, the steady-state flammability is bounded by the initial calculation and the hydrogen generation rate need not be reevaluated. 


\subsection{Summary and Conclusions}

The analyses presented in Section 3 based on the theory summarized in Section 2 have quantified the effects of the nine authorized globally waste-disturbing activities in terms of gas release potential as well as changes in waste behavior. The practical effects of each operation differ depending on the initial waste group assignment of a tank. The three waste groups are defined in Section 2.4.1. Waste Group A and B tanks store sufficient gas to make the headspace flammable if all of it were suddenly released; Waste Group C tanks do not. Waste Group A tanks exhibit spontaneous BDGREs, while tanks in Waste Group B do not.

This section summarizes the conclusions of the analyses by waste group. Section 4.1 summarizes the gas releases that could be induced by the nine globally waste-disturbing activities, and Section 4.2 discusses potential long-term changes in waste group classification. Section 4.3 lists cases where the hydrogen generation rate and steady-state flammability need to be addressed.

\subsection{Potential for Significant Gas Releases}

A waste-disturbing activity can cause or influence gas releases in three ways: 1) spontaneous BDGREs in Waste Group A tanks may be amplified, 2) BDGREs can be induced by the effects of the operation, and 3) the waste disturbance itself can produce substantial gas releases that can accumulate to flammability in the headspace if the ventilation rate is low or the release rate is rapid compared with the ventilation rate. Table 4.1 lists the potential gas releases for each globally waste-disturbing activity by waste group. Note that, though gas releases occur in Waste Group $\mathrm{C}$ tanks, they are inconsequential by definition because these tanks have insufficient retained gas to make their headspace flammable even if all the gas is released instantaneously.

Inducing or exacerbating BDGREs is the greatest concern in any globally waste-disturbing activity in DSTs. Any addition to a tank already exhibiting spontaneous BDGREs will increase the resulting headspace hydrogen concentration by decreasing the headspace and increasing the hydrostatic pressure on the gas. However, this was shown in Section 3.5.4 not to cause previously nominal BDGRES in the current Group A tanks to exceed the LFL for addition of water or caustic to the maximum waste level. Adding volume could move a Group $\mathrm{C}$ tank exhibiting BDGREs directly to Group A. BDGREs can also be induced as a result of lowering the neutral buoyancy gas fraction, $\alpha_{\mathrm{NB}}$, by suspending sediment in the supernatant (by mixer pump or airlift circulator operation), or by otherwise increasing the supernatant density (by waste or chemical additions).

In SSTs there is no mechanism for large spontaneous or induced gas releases. However, these tanks are typically passively ventilated, and a relatively slow gas release during saltcake dissolution retrieval, water addition, or saltwell pumping can potentially accumulate to eventually raise the headspace hydrogen concentration to the LFL in Group A or B tanks. 
Table 4.1. Potential Gas Releases During Globally Waste-Disturbing Activities

\begin{tabular}{|c|c|c|c|}
\hline \multirow{2}{*}{ Operation } & \multicolumn{3}{|c|}{ Waste Group } \\
\hline & A & B & $\mathbf{C}^{(\mathbf{a})}$ \\
\hline $\begin{array}{l}\text { Waste Removal } \\
\text { (DST) }\end{array}$ & BDGRE (depressurization) & $\begin{array}{l}\text { Late BDGRE }^{(b)} \\
\text { (depressurization) }\end{array}$ & $\begin{array}{l}\text { BDGRE } \\
\text { (depressurization) }\end{array}$ \\
\hline $\begin{array}{l}\text { Waste Addition } \\
\text { (DST) }\end{array}$ & $\begin{array}{l}\text { Amplified BDGRE (higher } \\
\text { pressure, smaller head- } \\
\text { space, potential added } \\
\text { sediment) }\end{array}$ & $\begin{array}{l}\text { BDGRE (lower } \\
\left.\alpha_{N B}\right)^{(c)}\end{array}$ & BDGRE (lower $\left.\alpha_{\mathrm{NB}}\right)^{(\mathrm{c})}$ \\
\hline $\begin{array}{l}\text { Saltwell pumping } \\
\text { (SST) }\end{array}$ & N/A & $\begin{array}{l}\text { Slow release } \\
\text { (uncovery, } \\
\text { depressurization) } \\
\end{array}$ & $\begin{array}{l}\text { Slow release } \\
\text { (uncovery, } \\
\text { depressurization) }\end{array}$ \\
\hline $\begin{array}{l}\text { Saltcake } \\
\text { dissolution (SST) }\end{array}$ & N/A & $\begin{array}{l}\text { Slow release } \\
\text { (dissolution) }\end{array}$ & $\begin{array}{l}\text { Slow release } \\
\text { (dissolution) }\end{array}$ \\
\hline $\begin{array}{l}\text { Water addition } \\
\text { (SST) }\end{array}$ & N/A & $\begin{array}{l}\text { Slow release } \\
\text { (dissolution) }\end{array}$ & $\begin{array}{l}\text { Slow release } \\
\text { (dissolution) }\end{array}$ \\
\hline $\begin{array}{l}\text { Water addition } \\
\text { (DST) }\end{array}$ & $\begin{array}{l}\text { Amplified BDGRE (higher } \\
\text { pressure, smaller } \\
\text { headspace) }\end{array}$ & $\begin{array}{l}\text { Slow release } \\
\text { (dissolution) }\end{array}$ & $\begin{array}{l}\text { Slow release } \\
\text { (dissolution) }\end{array}$ \\
\hline $\begin{array}{l}\text { Mixer pump } \\
\text { operation (DST) }\end{array}$ & $\begin{array}{l}\left.\text { BDGRE (lower } \alpha_{\mathrm{NB}}\right) \text {, large } \\
\text { disturbance }\end{array}$ & $\begin{array}{l}\text { BDGRE (lower } \alpha_{\mathrm{NB}} \text { ), } \\
\text { large disturbance }\end{array}$ & $\begin{array}{l}\text { BDGRE (lower } \alpha_{\mathrm{NB}} \text { ), } \\
\text { large disturbance }\end{array}$ \\
\hline $\begin{array}{l}\text { Airlift circulator } \\
\text { operation }(D S T)^{(\mathrm{e})}\end{array}$ & $\begin{array}{l}\text { BDGRE (lower } \alpha_{\mathrm{NB}} \text { ), large } \\
\text { disturbance }\end{array}$ & $\begin{array}{l}\text { BDGRE (lower } \alpha_{\mathrm{NB}} \text { ), } \\
\text { large disturbance }\end{array}$ & $\begin{array}{l}\text { BDGRE (lower } \alpha_{\mathrm{NB}} \text { ), } \\
\text { large disturbance }\end{array}$ \\
\hline $\begin{array}{l}\text { Chemical addition } \\
\text { (DST) }\end{array}$ & $\begin{array}{l}\text { Amplified BDGRE (higher } \\
\text { pressure, smaller } \\
\text { headspace) })^{(\mathrm{d})}\end{array}$ & $\begin{array}{l}\text { BDGRE (lower } \\
\left.\alpha_{N B}\right)^{(c)}\end{array}$ & BDGRE (lower $\left.\alpha_{\mathrm{NB}}\right)^{(\mathrm{c})}$ \\
\hline $\begin{array}{l}\text { Natural } \\
\text { evaporation (DST) }\end{array}$ & None & None & None \\
\hline $\begin{array}{l}\text { (a) Gas releases are } \\
\text { insufficient gas to ma } \\
\text { (b) Induced BDGRE } \\
\text { flammability potentic } \\
\text { (c) BDGREs can be } \\
\text { (d) Water and causti } \\
\text { (e) The only DSTs w }\end{array}$ & $\begin{array}{l}\text { listed for Group } \mathrm{C} \text { tanks but } \\
\text { ke their headspace flammable. } \\
\text { s, if any, are expected late } \\
\text { is minimum. } \\
\text { revented by adjusting the wast } \\
\text { addition do not cause current } \\
\text { th potentially operable airlift cir }\end{array}$ & $\begin{array}{l}\text { have no consequence b } \\
\text { n the process where he } \\
\text { addition to keep the buo } \\
\text { DGREs to exceed the LF } \\
\text { culators are currently clas }\end{array}$ & $\begin{array}{l}\text { ecause these tanks retain } \\
\text { dspace is maximum and } \\
\text { ancy ratio }<1 \text {. } \\
\text { sified in Waste Group C. }\end{array}$ \\
\hline
\end{tabular}

Analytical methods were developed in Section 3 to determine the conditions under which gas releases, specifically BDGREs, would occur. In most cases these analyses indicate that flammable conditions will not occur as a result. Where there is some potential for BDGREs, operations can be adjusted to avoid it in most cases. 


\subsection{Potential Changes in Waste Group Classification}

The waste group classification depends basically on whether a tank retains enough gas to make its headspace flammable if all of it were released suddenly and whether it experiences spontaneous BDGREs. The potential changes in waste group classification for the nine authorized activities are listed by initial waste group in Table 4.2. The retained gas condition is determined mainly by the headspace so any addition to a tank tends to move it to a higher group. A Group C tank experiencing BDGREs (e.g., SY-103) could move all the way into Group A by waste addition.

Table 4.2. Potential Changes in Waste Group from Globally Waste-Disturbing Activities

\begin{tabular}{|c|c|c|c|}
\hline \multirow{2}{*}{ Operation } & \multicolumn{3}{|c|}{ Waste Group } \\
\hline & $\mathbf{A}$ & B & $\mathbf{C}^{(\mathrm{a})}$ \\
\hline $\begin{array}{l}\text { Waste Removal } \\
\text { (DST) }\end{array}$ & B or $C$ & $\mathrm{C}$ & None \\
\hline Waste Addition (DST) & None ${ }^{(b)}$ & A (lower $\left.\alpha_{N B}\right)$ & $\begin{array}{l}\text { A or B (lower } \alpha_{\mathrm{NB}} \\
\text { smaller headspace) }\end{array}$ \\
\hline $\begin{array}{l}\text { Saltwell pumping } \\
\text { (SST) }\end{array}$ & N/A & $\mathrm{C}$ & None \\
\hline $\begin{array}{l}\text { Saltcake dissolution } \\
\text { (SST) }\end{array}$ & N/A & $\mathrm{C}$ & None \\
\hline Water addition (SST) & N/A & None & $\begin{array}{l}\text { B (smaller } \\
\text { headspace) }\end{array}$ \\
\hline Water addition (DST) & None $^{(b)}$ & None & $\begin{array}{l}\text { A or B (smaller } \\
\text { headspace) }\end{array}$ \\
\hline Mixer pump operation & None & None & None \\
\hline $\begin{array}{l}\text { Airlift circulator } \\
\text { operation }\end{array}$ & None & None & None \\
\hline Chemical addition & None ${ }^{(b)}$ & A (lower $\left.\alpha_{N B}\right)$ & $\begin{array}{l}\text { A or B (lower } \alpha_{\mathrm{NB}} \text {, } \\
\text { smaller headspace) }\end{array}$ \\
\hline Natural evaporation & None $^{(\mathrm{c})}$ & $\mathrm{C}^{(\mathrm{c})}$ & None $^{(\mathrm{c})}$ \\
\hline \multicolumn{4}{|c|}{$\begin{array}{l}\text { (a) Waste Group C tanks cannot move into a lower group, but removal of waste will decrease their } \\
\text { retained gas volume and potential for releases. } \\
\text { (b) Waste Group A tanks cannot move to a higher group, but addition of waste, water, or chemicals will } \\
\text { amplify their spontaneous BDGREs. } \\
\text { (c) Significant evaporation does not occur in tanks with a high salt concentration, which currently } \\
\text { includes all Group A tanks. This also prevents initiation of BDGRE behavior by evaporation. }\end{array}$} \\
\hline
\end{tabular}

Though many factors influence BDGRE behavior, the dominant parameter is the neutral buoyancy gas fraction, which depends on the ratio of supernatant density to bulk sediment density. Therefore, any operation that makes the supernatant more dense can potentially decrease the neutral buoyancy gas fraction, initiate BDGREs, and move a tank from Group B to Group A. However, initiating BDGREs in a Group C tank will not move it to Group A unless the headspace were simultaneously reduced sufficiently. Waste, water, or chemical addition 
proves to be the only activity capable of moving a tank to a higher waste group. Conversely, all of the waste removal activities can move a tank to Group C.

\subsection{Potential Changes in Hydrogen Generation}

The hydrogen generation rate depends on the amount of waste, specifically the liquid portion, the concentration of dissolved salts (reduces water radiolysis), TOC, and aluminum (increases thermolysis) in the liquid. Waste addition clearly causes an increase in the hydrogen generation rate, and any major removal of waste, especially liquid, will decrease it.

On the other hand, Hu et al. (2002) have calculated that even water additions that fill the tank to maximum capacity do not have a strong effect on-and probably decrease-hydrogen generation. The calculation assumed that the added water mixes only with the supernatant liquid with no solids dissolution. In general, the waste is not saturated with TOC and aluminum; however, many tanks are saturated in nitrite and nitrate. Thus water addition under the nodissolution assumption would tend to correctly reduce the TOC and aluminum concentrations, while conservatively reducing the concentrations of nitrite and nitrate, which would tend to remain constant with dissolution. Because water radiolysis is reduced by nitrate and nitrite in the solution, the hydrogen generation rate from radiolysis is higher using the no-dissolution assumption. Therefore, the effect of any water addition on hydrogen generation is encompassed by these analyses.

We expect that, because it is the reverse of water addition, evaporation should not significantly change the hydrogen generation rate. However, addition of caustic may or may not increase the hydrogen generation and should be evaluated. ${ }^{\text {(a) }}$

The changes in hydrogen generation rate resulting from the nine activities are listed in Table 4.3. Because the effect of an operation on hydrogen generation rate does not depend on the waste group, only one column is shown in the table.

Table 4.3. Changes in Gas Generation from Globally Waste-Disturbing Activities

\begin{tabular}{||l|c||}
\hline \multicolumn{1}{|c|}{ Operation } & Effect on Hydrogen Generation \\
\hline Waste removal (DST) & Decrease \\
\hline Waste addition (DST) & Increase \\
\hline Saltwell pumping (SST) & Decrease \\
\hline Saltcake dissolution (SST) & Decrease \\
\hline Water addition & None \\
\hline Mixer pump operation & Pump energy deposition issue \\
\hline Airlift circulator operation & None \\
\hline Chemical addition & Potential increase \\
\hline Natural evaporation & None \\
\hline \hline
\end{tabular}

(a) Personal communication with Albert $\mathrm{Hu}(\mathrm{CHG})$. 
Energy dissipation from one or more mixer pumps being run for long periods is an issue. Mixer pump operation can elevate the waste temperature considerably. Because hydrogen generation follows Arrhenius behavior (see Section 2.1), it is sensitive to temperature. A relatively short period of continuous mixer pump operation could easily increase the hydrogen generation rate by an order of magnitude. If the mixer pump must be run such that the waste temperature remains elevated for an extended period, the steady-state flammability hazard of the tank should be evaluated at the new temperature.

\subsection{Implications for Other Operations}

This report has treated nine specific globally waste-disturbing activities that are currently performed or proposed in SSTs and DSTs. In addition to these activities, there are many local waste-disturbing activities listed in the Final Safety Analysis Report (FSAR) (Cash 2000). These activities are discussed in Sections 4.4.1 in terms of the analysis and conclusions derived for the globally waste-disturbing activities. Some globally waste-disturbing activities that have not been analyzed are noted in Section 4.4.2. A brief summary is given in Section 4.4.3.

\subsubsection{Local Waste Disturbances}

The local waste-disturbing activities can be grouped into four broad categories according to the general type of disturbance involved. Each category is briefly examined below. No gas release associated with these activities is expected to create headspace hydrogen concentrations exceeding the action level (see the historical summary in Section 1.2.1), nor can any of them change a tank's waste classification. However, it is important to note that spontaneous BDGREs can occur at any time in Waste Group A tanks that have historically raised the hydrogen concentration near or above the action level (though less than $50 \%$ of the LFL).

Water lancing: operation of water lances or jets lowered vertically into the waste, usually to create a passage or cavity for installing other waste-penetrating equipment. Water lances range in design from a single vertically oriented nozzle that opens a 4-inchdiameter hole to the 42-inch cruciform lance that uses high-pressure jets to excavate for the SY-101 mixer pump in 1993. Lancing adds up to 2,000 gallons of water locally per the FSAR. This is less than $1 / 5$ of the 11,000-gallon threshold water volume that was predicted to cause a hazardous gas release by dissolution in Section 3.5.2.

Equipment installation: Examples include installation of mixer pumps and transfer pumps, saltwell screens and liquid observation wells, and thermocouple trees and multifunction instrument trees. Diameters range from less than 4 inches to about 40 inches. Installation involves pushing equipment vertically into the waste. This causes a local waste disturbance of approximately the same volume as that of the device below the waste surface. Water lancing is often a prerequisite for installation, or a water lance is designed into the base of the equipment to ensure that it penetrates the waste easily.

Equipment removal: Examples are removal of mixer pumps, sludge weights, air lances, liquid observation wells, thermocouple trees, and multifunction instrument trees. Removing equipment that has been installed in the waste for a long time requires rather 
extensive water flushes for decontamination, which adds to the direct waste disturbance of the removal itself. Flushes are not expected to approach the 11,000-gallon threshold for hazardous gas release calculated in Section 3.5.2.

Equipment operation: This involves operation of the ball rheometer, VFI, core sampling, densitometer readings, and liquid grab sampling. Running the validation probe inside a multifunction instrument tree or taking neutron or gamma logs in a liquid observation well are not considered because they occur inside a sealed volume and do not disturb the waste. Operation of waste-penetrating equipment is actually a combination of the installation and removal disturbances. However, because such equipment is designed specifically for operating in the waste, the disturbance attending its operation is typically less than that of devices intended for permanent installation. Measurement and sampling devices are typically of small diameter and flushes are small because they have been designed for easy decontamination.

\subsubsection{Other Global Waste Disturbances}

Gas releases expected during the U-107 saltcake dissolution proof-of-concept test and the S-112 saltcake dissolution demonstration project are covered in Section 3.4. Several more specific operations are described below that are not covered by the analyses in this document. These activities would require further definition and study to assess the potential hazard adequately. The main concern about gas releases during global waste disturbances is the amount of waste disturbed and its release rate. Given the retained gas volume fraction and assuming that the disturbance is sufficient to release most or all of gas, the volume of waste disturbed in a given time is a direct measure of the volume of gas released. If this is large compared with the headspace ventilation rate, high hydrogen concentrations can result. Therefore, planning these activities is aimed at controlling the waste disturbance to limit gas release to acceptable values. The list is not complete, but it may serve to illustrate the issues.

Sluicing: This operation has been performed for many years, most recently in 1999 in Tank C-106. It uses one or more high-flow, medium-pressure hydraulic jet(s) to mobilize the waste and move it to the inlet of a transfer pump, where the slurry is transferred to a DST. C-106 waste was sluiced using supernatant from the receiving DST AY-102 in a closed-loop system. In terms of the globally waste-disturbing activities discussed in this report, it represents a combination of mixing and saltcake dissolution that has the potential for large and rapid waste disturbances. Because sluicing cannot be performed through a supernatant layer, however, induced BDGREs would not be a concern. Sluicing to date has involved relatively shallow sediment layers that do not retain much gas. If performed in a Waste Group B SST, the potential for reaching the LFL would warrant development of a plan carefully specifying the amount of waste to be retrieved in each batch. If performed in a DST, sluicing would need to be preceded by supernatant decant that would release some of the gas and increase the headspace, possibly putting the tank into Waste Group C. If not, a plan limiting the batch size would also be required for these tanks. 
S-102 Fluidic Retrieval Demonstration: The system proposed for this project will use a power fluidic mixing and pumping system consisting of several air-driven charge vessels installed inside the tank to mobilize the waste. Relatively small quantities of water will be introduced to dissolve the soluble portion of the waste and mobilize the rest to allow it to be pumped from the tank. Again, the nature of the global disturbance represents a combination of dissolution and mixing with the potential for a large, rapid waste disturbance and gas release. Also, S-102 prior to saltwell pumping had one of the largest retained gas inventories of the SSTs that once populated the Flammable Gas Watch List (Hedengren et al. 2001). Because S-102 contains a mixture of sludge and saltcake, saltwell pumping may not remove all of the drainable interstitial liquid, and a large fraction of the initial gas inventory may remain. The potential for large waste disturbances plus the possibility for large retained gas volume indicates the operation should be planned carefully to control gas releases.

C-104 Retrieval Demonstration: This tank is proposed to be retrieved by a mobile retrieval system or "crawler" that will retrieve the waste by local mechanical or hydraulic means. The crawler will also carry a system to transfer the mobilized waste out of the tank. Depending on how the process is finally designed, the process may not classify as a globally waste-disturbing activity except in final result. Because this retrieval method is intended for sludge tanks where the waste is relatively insoluble, saltcake dissolution is not an issue. These tanks typically contain little gas, so they are mostly in Waste Group C. Given the probable characterization of the crawler as a local waste disturbance and the low gas volumes expected, gas releases are probably not of great concern for this project.

\subsubsection{Summary}

Considering the entire spectrum of waste-disturbing activities described above and those analyzed in Section 3 of this report, it is difficult to conceive of a larger or more rapid waste disturbance than operation of one or more large mixer pumps. Because these pumps were designed to have sufficient power to mobilize the entire waste volume and mix it with sufficient intensity to keep the sediment suspended uniformly, they can quickly release all of the gas in a tank. Waste retrieval operations will also release all of a tank's retained gas but will do so more gradually or locally, so it is easier for ventilation to keep up with the release. These combined factors indicate that mixer pump operation may be the bounding global waste disturbance. Past experience and the recent analyses could serve as a model for planning similar, but less aggressive, activities. 


\subsection{References}

Allemann RT, ZI Antoniak, WD Chavala, LE Efferding, JG Fadeff, JR Friley, WB Gregory, JD Hudson, JJ Irwin, NW Kirch, TE Michener, FE Panisko, CW Stewart, and BM Wise. 1994. Mitigation of Tank 241-SY-101 by Pump Mixing: Results of Testing Phases A and B. PNL-9423, Pacific Northwest Laboratory, Richland, WA.

Barker SA and DC Hedengren. 2002. Methodology and Calculations for the Assignment of Waste Groups for the Large Underground Waste Storage Tanks at the Hanford Site. RPP10006, CH2M HILL Hanford Group, Richland, WA.

Barker SA, WB Barton, DR Bratzel, M Epstein, PA Gauglitz, GD Johnson, SN Maruvada, CE Olson, ML Sauer, SE Slezak, CW Stewart, and J Young. 1999. Flammable Gas Safety Analysis Review. SNL-000198, Sandia National Laboratory, Albuquerque, NM.

Cash RJ. December 2000. Tank Farms Final Safety Analysis Report (FSAR). HNF-SD-WMSAR-067, CH2M HILL Hanford Group, Inc., Richland, Washington.

Carlson AB, PJ Certa, TM Hohl, JR Bellomy III, TW Crawford, DC Hedengren, AM Templeton, HS Fisher, SJ Greenwood, DG Douglas, and WJ Ulbricht Jr. 2000. Test Report, 241-AZ-101 Mixer Pump Test. RPP-6548, Numatec Hanford Corporation, Richland, WA.

CHG. 2001. "Chemistry Control Program." Tank Farms Technical Safety Requirements, Sect. 5.15. HNF-SD-WM-TSR-006 Rev. 2b, CH2M HILL Hanford Group, Richland, WA.

Conway JT. 1993. Hanford Waste Characterization Studies. DNFSB Recommendation 93-5, Defense Nuclear Facilities Safety Board, Washington, D.C.

Cuta JM, KG Carothers, DW Damschen, WL Kuhn, JA Lechelt, K Sathyanayarana, and LA Stauffer. 2000. Review of Waste Retrieval Sluicing System Operations and Data for Tanks 241-C-106 and 241-AY-102. PNNL-13319, Pacific Northwest National Laboratory, Richland, WA.

DOE/RL. 1996. Recommendation 93-5 Implementation Plan. DOE/RL-94-0001 Rev. 1, U.S. Department of Energy Richland Operations, Richland, WA.

Ecology, EPA, and DOE. 1996. Hanford Federal Facility Agreement and Consent Order, as amended. Washington State Department of Ecology, U.S. Environmental Protection Agency, and U.S. Department of Energy, Olympia, WA.

Estey SD and MD Guthrie. 1996. An Analysis of Parameters Describing Gas Retention and Release Behavior in Double-Shell Tank Waste. WHC-SD-WM-TI-755, Westinghouse Hanford Company, Richland, WA.

Estey SD, JL Huckaby, LA Mahoney, CW Stewart, and BE Wells. 2001. Process Control Plan for Single-Shell Tank Saltcake Dissolution Proof-of-Concept. RPP-7715 Rev. 1, CH2M HILL Hanford Group, Richland, WA.

Fort LA. 2001. Chemistry Control Program Quarterly Review of Double-Shell Tank Caustic Limits Report Third Quarter Calendar Year 2001. RPP-9592, CH2M HILL Hanford Group, Inc., Richland, WA. 
Fowler KD. 1995. Tank Farm Waste Compatibility Program. WHC-SD-WM-OSD-015 Rev. 1, Westinghouse Hanford Company, Richland, WA.

Gauglitz PA, LA Mahoney, DP Mendoza, and MC Miller. 1994. Mechanisms of Gas Bubble Retention. PNL-10120, Pacific Northwest Laboratory, Richland, WA.

Gauglitz PA, SD Rassat, MR Powell, RR Shah, and LA Mahoney. 1995. Gas Bubble Retention and Its Effects on Waste Properties: Retention Mechanisms, Viscosity, and Tensile and Shear Strength. PNL-10740, Pacific Northwest Laboratory, Richland, WA.

Gauglitz PA, SD Rassat, PR Bredt, JH Konynenbelt, SM Tingey, and DP Mendoza. 1996. Mechanisms of Gas Bubble Retention and Release: Results for Hanford Waste Tanks 241-S-102 and 241-SY-103 and Single-Shell Tank Simulants. PNNL-11298, Pacific Northwest National Laboratory, Richland, WA.

Hanlon BM. 2002. Waste Tank Summary Report for Month Ending August 31, 2002. HNF-EP0182, Rev. 173, CH2M HILL Hanford Group, Richland, WA.

Hedengren DC, KM Hodgson, WB Barton, CW Stewart, JM Cuta, and BE Wells. 2000. Data and Observations on Double-Shell Flammable Gas Watch List Tank Behavior. RPP-6655, CH2M HILL Hanford Group, Richland, WA.

Hedengren DC, TA Hu, MA Kufahl, DJ McCain, CW Stewart, JL Huckaby, LA Mahoney, and KG Rappe. 2001. Data Observations on Single-Shell Flammable Gas Watch List Tank Behavior. RPP-7249, CH2M HILL Hanford Group, Richland, WA.

Hetsroni G. 1982. Handbook of Multiphase Systems. Hemisphere Publishing Company, Washington, D.C.

Hu TA. 2002. Steady-State Flammable Gas Release Rate Calculation and Lower Flammability Level Evaluation for Hanford Tank Waste. RPP-5926 Rev. 2, CH2M HILL Hanford Group, Richland, WA.

Hu TA. 2000. Empirical Rate Equation Model and Rate Calculations for Hydrogen Generation for Hanford Tank Waste. HNF-3851 Rev. 1, Lockheed Martin Hanford Corp., Richland, WA.

Huckaby JL, LM Peurrung, and PA Gauglitz. 1999. Gas Releases During Saltwell Pumping: Interpretation of Operational Data. PNNL-13029, Pacific Northwest National Laboratory, Richland, WA.

Johnson GD, NW Kirch, RE Bauer, JM Conner, CW Stewart, BE Wells, and JM Grigsby. 2000. Evaluation of Hanford High-Level Waste Tank 241-SY-101. RPP-6517, CH2M HILL Hanford Group, Richland, WA.

Johnson GD, WB Barton, RC Hill, JW Brothers, SA Bryan, PA Gauglitz, LR Pederson, CW Stewart, and LM Stock. 1997. Flammable Gas Project Topical Report. WHC-SP-1193 Rev. 2, Lockheed Martin Hanford Corporation, Richland, WA.

Johnson GD, DC Hedengren, JM Grigsby, CW Stewart, JJ Zach and LM Stock. 2001. Flammable Gas Safety Issue Resolution. RPP-7771, CH2MHILL Hanford Group, Richland, WA. 
Mahoney LA, ZI Antoniak, JM Bates, and ME Dahl. 1999. Retained Gas Sampling Results for the Flammable Gas Program. PNNL-13000, Pacific Northwest National Laboratory, Richland, WA.

Mahoney LA, ZI Antoniak, WB Barton, JM Conner, NW Kirch, CW Stewart, and BE Wells. 2000. Results of Waste Transfer and Back-Dilution in Tanks 241-SY-101 and 241-SY-102. PNNL-13267, Pacific Northwest National Laboratory, Richland, WA.

Mahoney LA and CW Stewart. 2002. Review of the Technical Basis of the Hydrogen Control Limit for Operations in Hanford Tank Farms. PNNL-13933, Pacific Northwest National Laboratory, Richland, WA.

McCain DJ. 2000. Results of Vapor Space Monitoring of Flammable Gas Watch List Tanks. HNF-SD-WM-TI-797 Rev. 5, CH2M HILL Hanford Group, Richland, WA.

Meyer PA and CW Stewart. 2001. Preventing Buoyant Displacement Gas Release Events in Hanford Double-Shell Waste Tanks. PNNL-13337, Pacific Northwest National Laboratory, Richland, WA.

Meyer PA and BE Wells. 2000. "Understanding Gas Release Events in Hanford Double Shell Tanks." Proceedings of Waste Management 2000, Tucson, AZ

Norton JD and LR Pederson. 1994. Ammonia in Simulated Hanford Double-Shell Tank Wastes: Solubility and Effects on Surface Tension. PNL-10173, Pacific Northwest National Laboratory, Richland, WA.

Peurrung LM, SM Caley, and PA Gauglitz. 1997. Gas Release During Salt-Well Pumping: Model Predictions and Laboratory Validation Studies for Soluble and Insoluble Gases. PNNL11621, Pacific Northwest National Laboratory, Richland, WA.

Peurrung LM, LA Mahoney, CW Stewart, PA Gauglitz, LR Pederson, SA Bryan and CL Shepard. 1998. Flammable Gas Issues in Double-Contained Receiver Tanks. PNNL-11836, Rev. 2, Pacific Northwest National Laboratory, Richland, WA.

Rassat SD, PA Gauglitz, PR Bredt, LA Mahoney, SV Forbes, and SM Tingey. 1997. Mechanisms of Gas Retention and Release: Experimental Results for Hanford Waste Tanks 241$A W-101$ and 241-AN-103. PNNL-11642, Pacific Northwest National Laboratory, Richland, Washington.

Rassat SD, SM Caley, PR Bredt, PA Gauglitz, DE Rinehart, and SV Forbes. 1998. Mechanisms of Gas Retention and Release: Experimental Results for Hanford Single-Shell Waste Tanks 241$A-101,241-S-106$, and 241-U-103. PNNL-11981, Pacific Northwest National Laboratory, Richland, Washington.

Reid HC and JE Deibler. 1997. Earthquake-Induced Response and Potential for Gas Mobilization in Hanford Waste Tanks. PNNL-11668, Pacific Northwest National Laboratory, Richland, WA.

Simmons CS. 1996. Modeling Water Retention of Sludge Simulants and Actual Saltcake Tank Wastes. PNNL-10831, Pacific Northwest National Laboratory, Richland, Washington.

Stewart CW, ME Brewster, PA Gauglitz, LA Mahoney, PA Meyer, KP Recknagle, and HC Reid. 1996. Gas Retention and Release Behavior in Hanford Single-Shell Waste Tanks. PNNL-11391, Pacific Northwest National Laboratory, Richland, WA. 
Stewart CW and G Chen. 1998. Baseline Estimate of the Retained Gas Volume in Tank 241-C106. PNNL-11890, Pacific Northwest National Laboratory, Richland, WA.

Stewart CW. 2001. Gas Releases During Saltcake Dissolution for Retrieval of Single-Shell Tank Waste. PNNL-13597 Rev. 1, Pacific Northwest National Laboratory, Richland, WA.

Stock LM. 2000. The Chemistry of Flammable Gas. RPP-6664, CH2M HILL Hanford Group, Richland, WA.

Watrous RA, DJ McCain, and SA Barker. 2000. An Analysis of Tank and Pump Pit Flammable Gas Data in Support of Saltwell Pumping Safety Basis Simplifications. RPP-6334, CH2M HILL Hanford Group, Richland, WA.

Wells BE, JM Cuta, SA Hartley, LA Mahoney, PA Meyer, and CW Stewart. 2002. Analysis of Induced Gas Releases During Retrieval of Hanford Double-Shell Tank Waste. PNNL-13782, Pacific Northwest National Laboratory, Richland, WA. 


\section{Appendix}

\section{Checklist for Technical Peer Review}




\section{CHECKLIST FOR TECHNICAL PEER REVIEW}

Document Reviewed: PNNL-13781 Rev. 1, "Effects of Globally Waste-Disturbing Activities on Gas Generation, Retention, and Release in Hanford Waste Tanks," January 2003.

Scope of Review (e.g., document section or portion of calculation): Entire document

Yes No NA*

[ ] [ ] [x] 1. Previous reviews are complete and cover the analysis, up to the scope of this review, with no gaps.

[x] [ ] [ ] 2. Problem is completely defined.

[x] [ ] [ ] 3. Accident scenarios are developed in a clear and logical manner.

[x] [ ] [ ] 4. Analytical and technical approaches and results are reasonable and appropriate. (ORP QAPP criterion 2.8)

[x] [ ] [ ] 5. Necessary assumptions are reasonable, explicitly stated, and supported. (ORP QAPP criterion 2.2)

[x] [ ] [ ] 6. Computer codes and data files are documented

[x] [ ] [ ] 7. Data used in calculations are explicitly stated.

[] [] [x] 8. Bases for calculations, including assumptions and data, are consistent with the supported safety basis document (e.g., the Tank Farms Final Safety Analysis Report).

[x] [ ] [ ] 9. Data were checked for consistency with original source information as applicable. (ORP QAPP criterion 2.9)

[x] [ ] [ ] 10. For both qualitative and quantitative data, uncertainties are recognized and discussed, as appropriate. (ORP QAPP criterion 2.17)

[x] [ ] [ ] 11. Mathematical derivations were checked including dimensional consistency of results. (ORP QAPP criterion 2.16)

[x] [ ] [ ] 12. Models are appropriate and were used within their established range of validity or adequate justification was provided for use outside their established range of validity.

[x] [ ] [ ] 13. Spreadsheet results and all hand calculations were verified.

[x] [] [ ] 14. Calculations are sufficiently detailed such that a technically qualified person can understand the analysis without requiring outside information. (ORP $Q A P P$ criterion 2.5)

[x] [ ] [ ] 15. Software input is correct and consistent with the document reviewed.

[x] [ ] [ ] 16. Software output is consistent with the input and with the results reported in the document reviewed.

[x] [ ] [ ] 17. Software verification and validation are addressed adequately. (ORP QAPP criterion 2.6)

[ ] [ ] [x] 18. Limits/criteria/guidelines applied to the analysis results are appropriate and referenced. Limits/criteria/guidelines were checked against references. (ORP QAPP criterion 2.9)

[ ] [ ] [x] 19. Safety margins are consistent with good engineering practices.

[x] [ ] [ ] 20. Conclusions are consistent with analytical results and applicable limits.

[x] [ ] [ ] 21. Resulis and conclusions address all points in the purpose. (ORP QAPP criterion 2.3)

[ ] [ ] [x] 22. All references cited in the text, figures, and tables are contained in the reference list.

[ ] [ ] [x] 23. Reference citations (e.g., title and number) are consistent between the text callout and the reference list.

[ ] [ ] [x] 24. Only released (i.e., not draft) references are cited. (ORP QAPP criterion 2.1)

[ ] [ ] [x] 25. Referenced documents are retrievable or otherwise available. 
[ ] [ ] [x] 26. The most recent version of each reference is cired, as appropriate. (ORP QAPP criterion 2.1)

[ ] [ ] [x] 27. There are no duplicate citations in the reference list.

[] [1] $[\mathrm{x}]$ 28. Referenced documents are spelled out (title and number) the first time they are cited.

[ ] [ ] [x] 29. All acronyms are spelled out the first time they are used.

[ ] [ ] [x] 30. The Table of Contents is correct.

[] [ ] [x] 31. All figure, table, and section callouts are correct.

[x] [ ] [ ] 32. Unit conversions are correct and consistent.

[ ] [ ] [x] 33. The number of significant digits is appropriate and consistent.

[] [ ] [x] 34. Chemical reactions are correct and balanced.

[ ] [ ] [x] 35. All tables are formatted consistently and are free of blank cells.

[] [] [x] 36. The document is complete (pages, attachments, and appendices) and in the proper order.

[ ] [ ] $[x]$ 37. The document is free of typographical errors.

I] [ ] [x] 38. The tables are internally consistent.

[ ] [ ] [x] 39. The document was prepared in accordance with HNF-2353, Section 4.3, Attachment B, "Calculation Note Format and Preparation Instructions".

[x] [] [] Concurrence

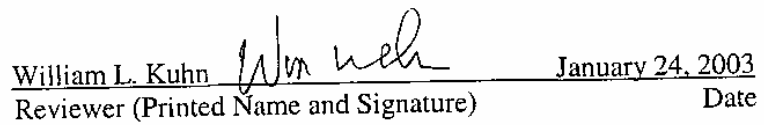

* If No or NA is chosen, provide an explanation on this form.

NA \# 1 - The entire document was reviewed.

NA \# 8 - Determination of consistency with Tank Farms safety basis was not part of this task.

NA \# 18 - Limits/criteria/guidelines were not applied in this task.

NA \# 19 - Safety margins were not determined in this task.

NA \# 22, 23, 24, 25, 26, 27, 28, 29, 30, 31, 32, 35, 36, 37, 38 - An editorial review was not performed as part of this technical review. However, an editorial review was performed by PNNL prior to issuance.

NA\# 34 - No specific chemical reactions are presented in the document.

NA\# 39 - CHG did not request this document to be prepared in accordance with HNF-2353, Section 4.3, Attachment B. 


\section{Distribution}

No. of

Copies

\section{Offsite}

Thomas E. Hicks

785 Valley Summit Dr.

Roswell, GA 30075

Dr. Scott Slezak

MS 0716

Sandia National Laboratory

Albuquerque, NM 87185-0716

BC Simpson

Portage Environmental

710 George Washington Way

Richland, WA 99352
No. of

Copies

14 CH2M HILL Hanford Group

SA Barker

R1-44

WB Barton

R2-11

CA Carro

R1-44

WL Cowley (3)

R1-44

JM Grigsby

R1-44

DC Hedengren

R3-73

TA Hu

R2-11

NW Kirch

R3-73

LJ Kripps

R1-44

DA Reynolds

R2-11

CHG Correspondence Control

TCSRC

H6-08

R1-10

16 Pacific Northwest National Laboratory

SQ Bennett

K7-90

JW Brothers (3)

K9-20

PA Gauglitz

K6-28

JL Huckaby

K7-15

WL Kuhn

$\mathrm{K} 7-15$

PA Meyer

K7-15

LA Mahoney

$\mathrm{K} 7-15$

CW Stewart (3)

K7-15

BE Wells

$\mathrm{K} 7-15$

J Young

K6-04

Information Release (2)

K1-06 